\title{
Cannabinoid Formulations and Delivery Systems: Current and Future Options to Treat Pain
}

\author{
Barbara Stella $^{1} \cdot$ Francesca Baratta $^{1} \cdot$ Carlo Della Pepa $^{1} \cdot$ Silvia Arpicco $^{1} \cdot$ Daniela Gastaldi $^{2} \cdot$ Franco Dosio $^{1}$ (i)
}

Accepted: 19 July 2021 / Published online: 4 September 2021

(C) The Author(s) 2021

\begin{abstract}
The field of Cannabis sativa L. research for medical purposes has been rapidly advancing in recent decades and a growing body of evidence suggests that phytocannabinoids are beneficial for a range of conditions. At the same time impressing development has been observed for formulations and delivery systems expanding the potential use of cannabinoids as an effective medical therapy. The objective of this review is to present the most recent results from pharmaceutical companies and research groups investigating methods to improve cannabinoid bioavailability and to clearly establish its therapeutic efficacy, dose ranges, safety and also improve the patient compliance. Particular focus is the application of cannabinoids in pain treatment, describing the principal cannabinoids employed, the most promising delivery systems for each administration routes and updating the clinical evaluations. To offer the reader a wider view, this review discusses the formulation starting from galenic preparation up to nanotechnology approaches, showing advantages, limits, requirements needed. Furthermore, the most recent clinical data and meta-analysis for cannabinoids used in different pain management are summarized, evaluating their real effectiveness, in order also to spare opioids and improve patients' quality of life. Promising evidence for pain treatments and for other important pathologies are also reviewed as likely future directions for cannabinoids formulations.
\end{abstract}

\section{Key Points}

Beneficial effects of cannabinoids formulations have been proved in clinical studies for several diseases.

Explore different administration routes to improve cannabinoid efficacy.

Efforts have been made to reduce pain and to improve quality of life of patients by designing appropriate formulations of cannabinoids.
Franco Dosio

franco.dosio@unito.it

1 Department of Drug Science and Technology, University of Turin, v. P. Giuria, 9, 10125 Turin, Italy

2 Department of Molecular Biotechnology and Health Sciences, University of Turin, Turin, Italy

\section{Introduction}

Although cannabinoids have attracted attention for centuries, the last few decades have brought new and scientifically well-founded insights into their therapeutic potential. The use of Cannabis sativa $\mathrm{L}$. for medical purposes, including herbal cannabis products and purified single active agents (mainly cannabinoids), has become increasingly available to patients in many countries in recent years and continues to evolve. The official introduction of pharmaceuticalgrade cannabis flowers for medicinal purposes has allowed physicians and pharmacists to legally prepare and prescribe cannabis-based products. Several countries throughout Europe have introduced specific regulations and guidelines regarding medicinal cannabis. Analogously, in Canada, the cannabis act has improved patient access, while, in the USA, the use of cannabis for medical purposes is legal in 36 states.

A growing number of US states now permit its administration for a plethora of conditions, including chronic and cancer-related pain, the nausea and vomiting caused by chemotherapy, the spasticity caused by multiple sclerosis and paraplegia, sleep disorder, psychosis, Tourette's syndrome, anxiety disorder and glaucoma [1]. 
The specific focus of this review is the application of cannabinoids in pain treatment, in order to spare opioids and other drugs, and their involvement in better patient quality of life.

According to the Kyoto protocol of the International Association for the Study of Pain, pain has been defined as an 'unpleasant sensory experience associated with actual or potential tissue damage or described in terms of such damage' [2]. Chronic pain, in particular, is a common, complex and distressing problem that has a deep impact on individuals and society. In the CDC survey in the year 2019, 20.4\% of US adults had chronic pain and $7.4 \%$ of adults had chronic pain that frequently limited life or work activities (referred to as high-impact chronic pain) [3]. The latest report on the Global Burden of Diseases, Injuries and Risk Factors identified low back pain among the top ten causes for all ages [4]. Pain may also be the major symptom in several conditions, such as fibromyalgia, endometriosis, inflammatory bowel disease, multiple sclerosis, rheumatoid arthritis and cancer. Although opioids do have a foundational role in the management of pain, non-opioid analgesic alternatives should be investigated as a means to increase the arsenal available to pain physicians. Furthermore, the opioid overdose crisis in the USA and the risks and harm that can arise from the misuse of medicines for pain management are concerning.

Despite the significant increase in the number of publications on medical cannabis-related topics, much of the published literature and funding for research on cannabis is still dedicated to surveys of willing cannabis users and the harm that the drug causes [5]. The medical community has been reticent to embrace cannabis as a medicine for many reasons, including decades of misinformation, poorly designed studies and lack of quality, standardised products and efficient formulations. At the same time, the market was flooded with many cannabinoid-based products, and many forms publicised medicinal value that has not been scientifically evaluated. These unregulated products, labelled as cannabinoids, may thus be a serious health hazard.

Nevertheless, the vast interest in the subject is clearly evident upon an exploration of the increasing number of articles and filed patents that evaluate the real efficacy of these substances, which were frequently used in the past as traditional medicines.

In order to select and suggest the most promising formulations and administration routes for pain treatment, it is important to have a wide overview of the cannabinoid formulations that have recently been explored and tested (years 2018-2021) by industry and research groups. Previous approaches and data can also be found in our previous review [6]. Several formulations have been proposed for other therapeutic areas (anorexia, seizure, rare syndromes), and knowledge of these is necessary if we are to understand their potential effectiveness and limits, and thus their real value as potential formulations for pain treatment and to improve the patient's quality of life.

For this reason, we will start by evaluating the galenic preparations that include the full spectrum of active ingredients in cannabis inflorescences. The complexity of this approach will be discussed as will the interesting, although hotly debated, matter of the entourage effect, which describes the synergic action of different components in the vegetable source.

Then, due to the huge amount of research published and patented on cannabinoid applications in recent years, we will describe studies that are in advanced clinical evaluations, categorised by administration routes, to allow the reader to compare the formulation approaches that are being developed by industry. Furthermore, the more recent development of micro- and nano-formulations, which were developed to deliver single cannabinoid or complex extracts, will also be presented.

Nanoscale designed formulations have been extensively studied in recent decades for compounds with high lipophilicity, providing significant advantages such as improving solubility, optimising their release profiles and increasing bioavailability, and have achieved important successes. Consequently, this can lead to the development of convenient administration routes, lower toxicity and fewer side effects, improved biodistribution and extended drug life cycles [7].

Although only preclinical data are available for the majority of the advanced technologies, some well-established formulations begin to be evaluated in clinical trials.

Tables will be used to summarise the relevant involvement of industries in developing safe and efficient cannabinoid-based medicines and, in particular, define the recent ongoing clinical tests focused on pain-related treatments.

\section{Pain and Role of Cannabinoids}

Pain is the result of a series of ample interactions (nervous system, metabolic, immunological responses), modulated by various factors (environmental, cultural, physical, affective), that are capable of generating specific characteristics for each individual.

Generally, it is possible to identify three different types of pain according to the origin of the stimulus: structural mechanical nociceptive pain, inflammatory nociceptive pain and neuropathic pain (peripheral and central).

Nociceptive pain develops at the peripheral level, following the stimulation of nociceptors, which send the stimulus through the nerve fibres of the somato-sensory system to the spinal cord and then to the thalamus and cerebral cortex. Structural mechanical nociceptive pain originates from nociceptors subjected to high intensity stimuli (above the sensibility threshold). Inflammatory nociceptive pain is, instead, 
caused by a sensitisation of the peripheral nociceptors by the mediators of the inflammatory process, which respond, in this case, to low intensity stimuli (below threshold) or even in the absence of stimuli (spontaneous pain).

Neuropathic pain is the consequence of damage to the peripheral nerves (peripheral neuropathic pain) or to the central nervous system (central neuropathic pain), which can cause the abnormal activity of the genesis of the impulses and is characterised by an almost complete lack of correlation between the entity of the peripheral stimulus and the painful sensation caused.

Acute pain corresponds to ongoing tissue damage, has a limited duration over time and is accompanied by neuroendocrine, emotional, and behavioural responses and, if not managed correctly, can evolve into chronic pain.

Chronic pain, which by convention lasts more than 3 months, may no longer have connections with the initial cause, so much so that the pain itself becomes a "disease".

Chronic pain has been linked to numerous physical and mental conditions and is one of the most common reasons why adults seek medical care. It is also linked to restrictions in mobility, daily activities and lost productivity [8]. Chronic pain reduces quality of life, causes the onset of anxiety and depression and, in some social contexts, dependence on opioids. The estimate of prevalence of chronic pain ranges from 11 to $40 \%$ [9-11].

Nociplastic pain, as defined by the International Association for the Study of the Pain (IASP-PAIN) in 2017, is caused by an alteration of nociception, although there is no clear evidence of actual or potential tissue damage causing the activation of peripheral nociceptors or evidence of disease or injury to the somatosensory system causing pain. This new term replaces other nosological entities such as dysfunctional pain or medically unexplained somatic syndromes and groups all the syndromes where chronic primary pain is present.

The most common nociplastic pain syndromes are chronic widespread pain, fibromyalgia, chronic low back pain of unknown causes, chronic visceral pain syndromes and others. This type of pain can occur in isolation but in these pathologies, pain is often accompanied by other symptoms such as fatigue, sleep disturbances, hypersensitivity to external stimuli, cognitive impairment and mood disturbance [12].

Recognising this type of chronic pain and the underlying pathogenetic mechanisms can help define a suitable drug treatment such as $N$-methyl-D-aspartate receptor antagonists and cannabinoids aimed at treating the sensitisation of central nervous system or peripheral nerve tissues.

There is no doubt about the historic use of plant-derived cannabinoids for inflammation and pain relief in many cultures since ancient times. While the explanation for their anti-inflammatory and analgesic effects remained unidentified until the discovery of the endocannabinoid system, the rationale for their use now seems to be clearer; it is known that the location and signalling of cannabinoid receptors are the basis for these effects.

The identification of D-9-tetrahydrocannabinol (THC), the compound responsible for the plant's psychotropic effects, paved the way for the discovery of the endocannabinoid system in vertebrates.

Cannabinoid receptors can be viewed in two ways: the first "classical" view is that there are only two recognised receptors, $\mathrm{CB} 1$ and $\mathrm{CB} 2$, which are members of the seven transmembrane G-protein coupled receptors; while, in a more ample view, cannabinoid and endocannabinoid compounds can activate a more widespread range of receptors.

Endogenous cannabinoids are fatty acids with 20 carbon atoms (eicosanoids) capable of selectively binding to the $\mathrm{CB} 1$ and $\mathrm{CB} 2$ receptors, the latter is mainly present in immune system cells. $N$-arachidonyl-ethanolamine better known as anandamide (AEA) was the first endogenous molecule isolated and identified in 1992 in the pig brain, followed by the discovery in 1995 of 2-arachidonoylglycerol (2-AG).

Endocannabinoids are synthesised by neurons, following the depolarisation of the membrane and the intracellular increase in calcium levels. The synthesis takes place starting from membrane phospholipids, precursors that after enzymatic hydrolysis, by means of two enzymes $\mathrm{N}$-acyl phosphatidylethanolamine phospholipase D (NAPE-PLD) and diacylglycerol lipase (DAGL), respectively, release the endocannabinoids AEA or 2-AG from the pre- or postsynaptic membrane, into the synaptic space. They are molecules that have a local effect and a short bioavailability as they are rapidly degraded by specific enzymes for endocannabinoids (fatty acid amide hydrolase, FAAH, and monoacylglycerol lipase, MAGL) (Fig. 1).

After release by the postsynaptic neurons, endogenous cannabinoids can travel retrograde along the synaptic cleft, also binding to presynaptic cannabinoid receptors where the $\mathrm{CB} 1$ receptors are most prevalent. The activation of cannabinoid $\mathrm{CB} 1$ receptors involves the inhibition of the activity of adenylate cyclase, with less production of the second messenger cAMP, initiates the closure of the $\mathrm{Ca}^{2+}$ channels, inhibiting the entry of $\mathrm{Ca}^{2+}$ ions, and opens the potassium channels causing hyperpolarisation of the membranes. Endocannabinoids, that can be defined neuromodulators, through this influence on ion channels, can inhibit the release of neurotransmitters from axons, interacting in particular with GABA-ergic, serotonergic, glutamatergic, and dopaminergic transmissions having an important role in some forms of short- and long-term synaptic plasticity. In addition, activation of some kinases may also occur, including mitogen-activated protein (MAP) kinases (Fig. 1). 


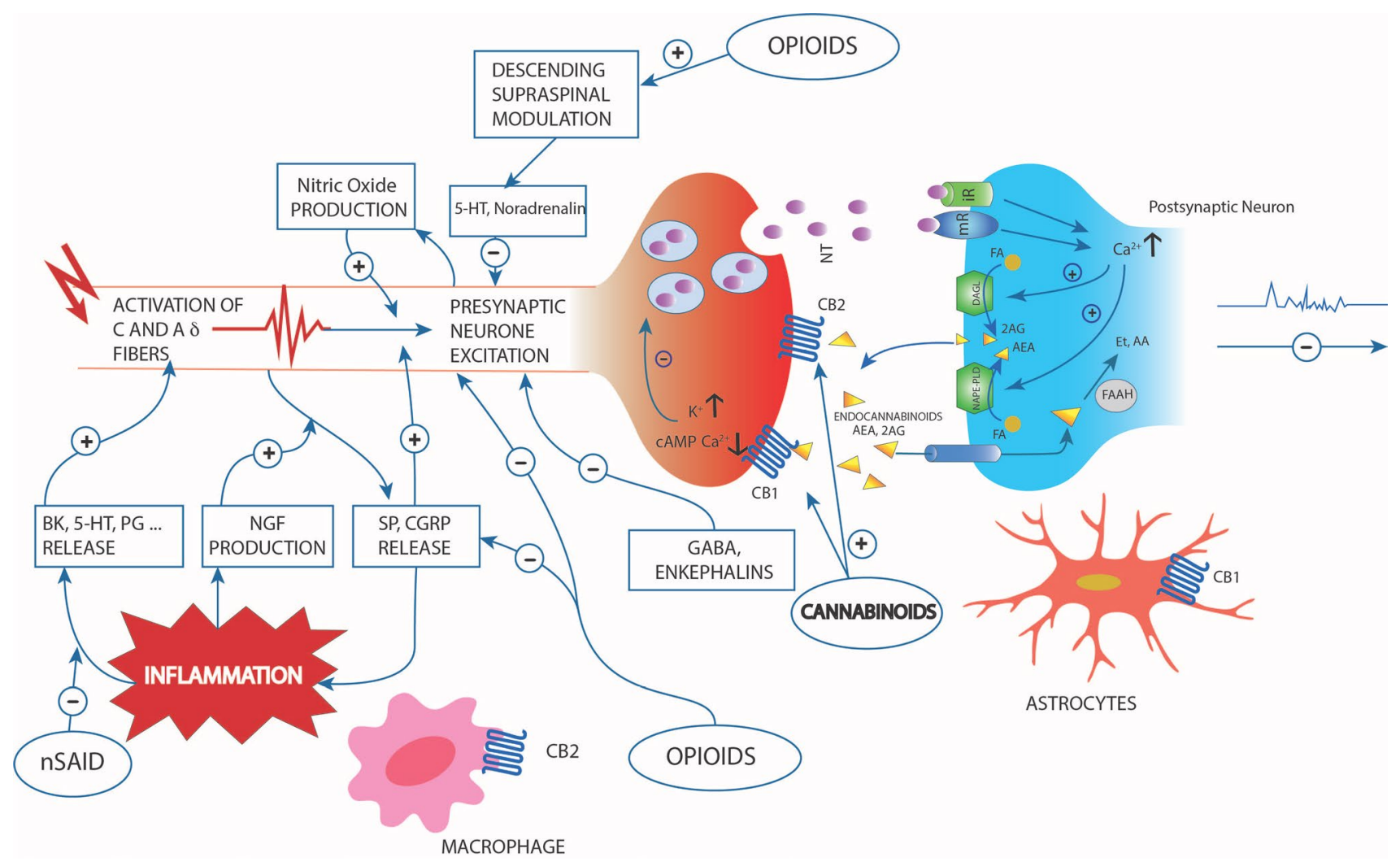

Fig. 1 Simplified scheme representing the pathogenesis of pain following inflammatory disease or nociceptive stimulus, the cytokines involved in the process, the descending supraspinal modulation and the relive neurotransmitters and endocannabinoid retrograde signalling mediated synaptic transmission. Endocannabinoids are produced from postsynaptic terminals upon neuronal activation. Natural and synthetic cannabinoids act like the two major endocannabinoids shown in the scheme: anandamide (AEA) and 2-arachidonolglycerol (2-AG). Endocannabinoids, synthesized starting from fatty acids (FA) through diacylglycerol lipase (DAGL) and $N$-acyl phosphatidylethanolamine phospholipase D (NAPE-PLD) readily cross the membrane and travel in a retrograde fashion to activate CB1 and CB2 located in the presynaptic terminals. Activated CB1 will then inhibit neurotransmitter (NT) release through the suppression of calcium influx. NT can bind to ionotropic (iR) or metabotropic (mR) receptors. 2-AG

The endocannabinoid system is involved in a large number of physiological processes, including motor control, memory and learning, pain perception, regulation of energy balance, and food intake. Other functions of the endocannabinoid system, in normal physiology, could be related to endocrine functions, vascular responses, modulation of the immune system and neuroprotection [13-15].

Cannabinoid-induced antinociception acts on the various levels of the pain sensory pathway, activating the antinociceptive pathways that are mediated by cannabinoids. The activity at the CB1 receptor system increases in response to noxious stimuli. Indeed, the CB1 receptors have been identified on primary afferent neurons on both type $\mathrm{C}$ and type $A \beta / \delta$ fibres, dorsal root ganglia, the spinal and terminal is also able to activate CB1 located in astrocytes. Although endocannabinoid retrograde signalling is mainly mediated by 2-AG, AEA can activate presynaptic CB1 as well. Fatty acid amide hydrolase (FAAH) found in postsynaptic terminals is responsible for degrading AEA to AA and ethanolamine (Et). Inflammation led to release of biochemical mediators (bradykinin (BK), serotonin (5-HT), prostaglandins etc) and the up-regulation of pain mediator nerve growth factor (NGF). The substance P (SP) and calcitonin gene-related peptide (CGRP) vasoactive neuropeptides, released from sensory nerve, also have a role in inflammation. The interaction with opioids, cannabinoids and nonsteroidal anti-inflammatory drugs are also represented. Adapted from Bruni et al. [6]; permitted reproduction under the terms and conditions of the Creative Commons Attribution (CC BY) license (https://creativecommons.org/licenses/by/4.0/).

intrinsic neurons of the dorsal horn of the spinal cord and neurons projecting to the brain (medulla rostral ventromedial, periaqueductal grey matter, amygdala, and thalamus).

At the spinal level, the analgesic effect of cannabinoids seems to be obtained from the activation of the OP2 opioid receptor. In fact, the analgesic effect is cancelled by the intrathecal administration of OP2 receptor antagonists. Periaqueductal grey matter (PAG) is one of the areas that is most strongly involved in mediating cannabinoid analgesia. This may be due, in part, to the direct inhibition of the release of GABA in this brain area and medulla, and, in part, may be related to the inhibition of glutamate release. In fact, the hyperalgesia induced by the CB1 antagonist is attenuated by the administration of NMDA receptor antagonists [46, 
47]. Cannabinoids activate a midbrain circuit to reduce pain sensations in the ventromedial rostrum (RVM) medulla [49]. In fact, the inactivation of this region prevents cannabinoid analgesia.

The antinociception induced by cannabinoids, at the level of the descending pathway, may be related, at least in part, to the release of norepinephrine. In fact, it has been shown that the antinociceptive effect can be attenuated by the administration of yohimbine but is not influenced by methysergide. Another analgesic mechanism that is induced by cannabinoids may be mediated via the interaction of dopamine and D2 receptors as it is enhanced by agonists and attenuated by selective antagonists. In addition, the cannabinoid and opioid systems show synergism that is mediated by receptors at the spinal and supraspinal levels. At the spinal level, cannabinoids activate the OP2 receptor, while opioids activate the OP3 and OP1 receptors. At the supraspinal level, synergism occurs via the activation of the OP3 receptor, indicating that analgesia from morphine, which is mainly mediated by the OP3 receptor, can be increased by cannabinoids through the activation of OP2 receptors. This synergism explains the reduction in opioid dosage when used in combination with cannabinoids. THC is capable of reducing the minimum effective dose of morphine by $55 \%$, methadone by $75 \%$ and codeine by $96 \%$ [52]. On the other hand, the cannabinoid system is larger and occupies more brain areas than the opioid system. However, the absence of specific receptors in the brain areas that control breathing, unlike those of morphine, prevents the risk of respiratory depression.

\subsection{Other Potential or Approved Therapeutical Uses of Cannabinoids}

In order to allow the reader to have a global view of the cannabinoid formulations, which have notably flourished in recent years, we also briefly describe other relevant therapeutic areas of application.

Therapeutic properties of cannabis plant have been known from ancient times and although, in most cases, results cannot be considered definitive and there are currently no pathological conditions for which cannabinoids can be considered the first-line treatment, the literature increasingly reports studies relating to the medical use of cannabis and cannabinoid for relevant diseases but also for the patients' well-being.

Anticancer potential activity of cannabinoids derived by observation that alterations of $\mathrm{CB} 1$ and $\mathrm{CB} 2$ expression levels and/or function have been observed in cancers [16]. Antineoplastic activity of cannabinoids demonstrated inhibition of growth of several cancer cell lines, and promising results have been obtained in animal cancer models (lung, breast and prostate and blood cancers). Nevertheless, highly heterogeneous cannabinoid receptor expression has been reported in several cancers, which it seems, are not ubiquitously expressed in all patients [17]. Regarding glioblastoma, the most frequent and aggressive malignant brain tumour, it found that elevated CB2 expression correlated with a higher degree of tumour malignancy. This led to the hypothesis that cannabinoids may exhibit effects against glioblastoma and this was confirmed by several in vivo studies [18].

Multiple sclerosis is an immune-mediated neurological disease, which leads to prolonged and progressive physical, psychological and cognitive disability. Chronic central neuropathic pain and spasticity are common distressing symptoms that cause disability and a lower quality of life [19]. It has been demonstrated that cannabinoids and the endocannabinoid system have a role in reducing spasticity in animal models, although recent clinical reviews concluded that cannabinoids may have a modest effect in the management of pain and spasticity in multiple sclerosis [20].

Cannabis galenic preparations as well as single purified cannabinoids can also be prescribed for the treatment of several neuropsychiatric conditions, such insomnia, depression and anxiety, attention deficit/hyperactivity disorder, autism, cluster headaches and, restless legs syndrome and Tourette's syndrome [1, 21, 22].

Emerging cannabinoid roles have recently appeared for management of associated neuropathological alterations in Alzheimer disease [23].

Important clinical results have been reported for cannabinoids in treatment of paediatric epilepsy. Therapeutic use of cannabis in treatment of infantile spasms has been documented since 1840 but it was only in 2018 that cannabidiol (as Epidiolex marketed medicine) was approved by the FDA and then by the EMA as an add-on antiepileptic drug in children with Dravet syndrome and Lennox-Gastaut syndrome (serious epileptic encephalopathies, generally resistant to treatment). The efficacy of this treatment in reducing seizures frequencies and in improving the quality of life in childhood epilepsy was shown in recent systematic reviews and was summarised by Raucci et al. [24].

The best way to establish a correlation between cannabis use and well-being is to measure the health-related quality of life (HRQoL), and this should be performed using validated questionnaires [25, 26]. HRQoL is defined as "those aspects of self-perceived well-being that are related to or affected by the presence of disease or treatment" [26, 27].

Considering that chronic diseases, due to their long duration, can negatively affect HRQoL, this parameter should be taken into consideration in clinical studies [28]. Some authors have also hypothesised that the use of cannabis may lead to a reduction in conventional therapies, which normally show several adverse effects. Therefore, an improvement in HRQoL could also be linked to the decrease in adverse drug reactions $[28,29]$. 
HRQoL is the most important target for patients in palliative cancer therapy. In palliative care, cannabinoids are usefully administered as antiemetics in cases of the nausea and vomiting caused by chemotherapy or radiotherapy [30]. The antiemetic drugs used to combat nausea and vomiting are not always effective and can have side effects on the central nervous system, particularly by causing sedation. The action of cannabinoids on CB1 localised in the dorsal vagal complex of the brainstem is considered to be responsible for the antiemetic activity of cannabinoids. There is evidence that cannabinoids are more effective at attenuating nausea, and FDA-approved dronabinol has therapeutic uses in the USA for nausea associated with cancer chemotherapy [31].

Furthermore, cannabinoids have the ability to counteract anorexia cachexia and weight loss, as they can act as an appetite stimulant in patients undergoing chemotherapy [32].

The reason for this effect lies in the presence of CB1 receptor in the hypothalamus, the region of the brain that controls food intake. Numerous studies have reported the role of cannabinoids in appetite stimulation, resulting in weight gain, when administered orally at low or moderate doses, with minimal side effects.

Another promising application of cannabinoids is the therapy of inflammatory bowel disease, especially Crohn's disease. Many gastrointestinal processes can be affected by cannabinoids because their receptors are distributed throughout the human gastrointestinal tract (CB2 is generally expressed by immune cells and the peripheral nervous system, while CB1 is more widely expressed in enteric nervous system, epithelial cells, myenteric and submucosal plexuses and adjacent to motoneurons) [33]. In particular, CBD appears to be an attractive therapeutic option, also due to lack of central effects. Nevertheless, although many patients could benefit from cannabinoid treatment, particularly when measuring HRQoL, at the moment, few clinical studies have been completed.

It is important to remember that cannabis-derived products (particularly THC, dronabinol, nabilone), are also associated with relevant short-term adverse effects including drowsiness, dizziness, confusion, hallucinations, euphoria, nausea and vomiting, and diarrhoea as reported in clinical trials [34]. These adverse effects remain similar across the diverse patient populations and to mitigate these effects, individual dose titration may be considered.

\section{Data Sources and Search Strategy}

An electronic search was conducted using several search engines: the Chemical Abstract Service Scifinder, which indexes Medline and Chemical Abstract reference databases and provides a wide range of international patents; Springer Nature AdisInsight, which also brings together information on drugs in commercial development, deals and key clinical trials; and Scopus (Elsevier). The following clinical trial databases were also explored: clinicaltrials.org; anzctr.org. $\mathrm{au}$; and eudract.ema.europe.eu. To complete the investigation into patent sources, the Questel Orbit Database was also used. Searches were carried out in the period January-April 2021 with check for updates.

Professional organisation guidelines, documents from international medicine institutions (Food and Drug Administration [FDA] European Medicines Agency [EMA], Government of Canada), and academic websites were also utilised, as appropriate.

Regarding selection criteria, the studies were included in the review if they described the use of a cannabis-based product and/or medication (e.g., dronabinol, nabilone, Nabiximols, cannabidiol, $\Delta$ 9-tetrahydrocannabinol), singularly or in combination with the qualifying condition and its synonyms; cannabinoid formulations or cannabinoid dosage forms - in different administration routes. Patents were also extracted using the name of the applicant companies. Furthermore, information related to cannabis for medical purposes, standards and analytical methods were extracted from German, Swiss and US pharmacopoeias.

Sources of information on cannabinoid use in the treatment of pain were extensively analysed, although other common disease states were included. The search period spanned mainly from 2018 through to April 2021, for the scientific articles, while for patents, the search could cover a broader period (last decade), as well as more relevant reviews. The articles included were limited to those in the English and German languages and, principally, to studies conducted in humans.

The authors of this review chose to include cannabisbased medications and explore the wide range of products in industrial development as well as galenic preparations. Thus, basic information and descriptions will be detailed. However, cannabis preparations that are identified as food, food supplements, cosmetics and for recreational use, generally referred to as cannabis-based products without a standardised pharmaceutical form, are outside the scope of this article.

\section{Principal Cannabis Active Agents}

Cannabis sativa $\mathrm{L}$. contains a large range of identified compounds, although the main active principles are the cannabinoids. Phytocannabinoids and terpenoids are synthesised in cannabis in secretory cells inside glandular trichomes and are most highly concentrated in unfertilised female flowers prior to senescence [35]. Over 120 different phytocannabinoids have been isolated [36], and of these, $\Delta$ 9-tetrahydrocannabinol $(\triangle 9$-THC, hereafter THC; $C A S$ 
Fig. 2 Structures of the principal cannabinoids described in the text<smiles>CCCCCc1cc(O)c2c(c1)OC(C)(C)[C@H]1CCC(C)=CC21</smiles>

THC<smiles>CCCCCCC(C)(C)c1cc(O)c2c(c1)OC(C)(C)[C@@H]1CCC(=O)C[C@H]21</smiles>

Nabilone<smiles>CCCCCCC(C)(C)c1cc(OC)c([C@H]2C=C(CO)C3CC2C3C)c(OC)c1</smiles>

HU-308<smiles>C=C(C)[C@H]1CCC(C)=C[C@H]1c1c(O)cc(CCCCC)cc1O</smiles><smiles>C=C(C)[C@H]1CCC(C)=CC1C1=C(O)C(=O)C(CCCCC)=C(NCc2ccccc2)C1=O</smiles>

VCE -004.8<smiles>CCCCCC1=C(NCC)C(=O)C(C/C=C(\C)CCC=C(C)C)=C(O)C1=O</smiles>

VCE -003.2<smiles>C=C(C)[C@H]1CCC(C)=CC1c1c(O)cc(CCCC(C)(C)C)cc1OC(=O)C(NC(=O)CCC(=O)O)C(C)C</smiles>

1972-08-3) and cannabidiol (CBD; CAS 13956-29-1) are the most abundant and widely studied (see structures in Fig. 2).

The following are expressed in lower amounts, although they are therapeutically promising in some cases: cannabinol (CBN; CAS 521-35-7), cannabigerol (CBG; CAS 25654-31-3), cannabichromene (CBC; CAS 20675-518), ), cannabidivarin (CBDV; CAS 24274-48-4), cannabigerivarin (CBGV; CAS 55824-11-8), cannabichromevarin (CBCV; CAS 57130-04-8), $\Delta$ 9-tetrahydrocannabivarin ( $\triangle 9$-THCV; CAS 31262-37-0), and their related acids [37].

While cannabinoids are produced by the cannabis plant as carboxylic acids, the carboxyl group is not very stable and is easily lost under the influence of light, heat, or prolonged storage.
Terpenes are another class of organic components that are widely represented in the plant (approximately 140 different types). Terpenes that are often encountered in cannabis varieties include the monoterpenes myrcene, $\alpha$-pinene, $\beta$-pinene, limonene, terpinolene, while $\alpha$-caryophyllene, humulene and others, present in traces, represent the sesquiterpene fraction [38].

Besides cannabinoids and terpenes, cannabis plants produce interesting polyphenolic compounds called flavonoids as well as steroids, pigments and other substances.

From a pharmaceutical point of view, it is important to remember that cannabis is hyper-accumulator for heavy metals. Furthermore, microbial contaminants, pesticide residues, and harsh extraction solvents can be also present in the extracts. Therefore cultivation, extraction and purification steps and analytical methods must meet the current Good 
Manufacturing Practice standards. On the contrary, cannabinoids produced by synthesis start from a characterised starting material and yield high purity products. Frequently cannabinoids can also be produced by semi-synthesis starting from precursors extracted from hemp further modified by chemical reactions (e.g., dronabinol). Furthermore, several novel synthetic derivatives of phytocannabinoids have been designed and tested in recent years.

From a physicochemical point of view, pharmacologically active cannabinoids are classified as Class II, according to the Biopharmaceutics Classification System (BCS) for oral drugs, as they have very low water solubility and high lipophilicity; CBD, (12.6 mg/L, $\log P$ 6.3, pKa 9.29), THC (28.0 $\mathrm{mg} / \mathrm{L}, \log P$ 6.97, $\mathrm{p} K \mathrm{a} 10.6)$.

Regarding its elimination route, as outlined by the Biopharmaceutics Drug Disposition Classification System (BDDCS), cannabinoids belong to Class II, which indicates compounds that are poorly water soluble and that are extensively eliminated by metabolism [39]. Indeed, the important first-pass effect (liver metabolism) limits the oral dose of CBD that reaches systemic circulation by about $75 \%$.

Several recent reviews have thoroughly described the pharmacokinetic and biodistribution behaviour of cannabinoids [40-43].

\section{Galenic Preparations of Cannabis Extracts}

In recent years, the official introduction of pharmaceutical grade cannabis inflorescences for medicinal use has offered medical doctors the opportunity to prescribe, and for pharmacists to prepare, cannabis-based formulas [44].

In order to provide patients legal access to medical cannabis, an ever increasing number of countries have introduced specific regulations on this topic [45]. In Europe, for example, the United Kingdom (UK), Netherlands, Italy, Germany, the Czech Republic, Croatia and Finland have introduced specific regulations and guidelines regarding medicinal cannabis [44]. The same is true globally in Canada, the USA, Israel and Australia, for example [45]. The regulatory framework for cannabis as a pharmaceutical product varies widely across the world, but in general, the laws in force in the different contexts specify the medical conditions, including pain, for which cannabis can be prescribed and which forms of administration are allowed [44].

The inflorescences available for medical use are of different varieties and are characterised by a different content of active molecules. In this regard, it is important to underline that the contents of THC and CBD are usually reported on the label by manufacturers. Furthermore, the percentages of THC and CBD must be understood as referring to the "total", i.e. the sum of the acidic and the decarboxylated molecules [46].

The inflorescences, which can be marketed in raw form or following granulation, can be smoked, vaporised using specially developed devices, or can be subjected to extraction processes to provide preparations for oral administration [44].

The literature reports on the different extraction methods that can be used on cannabis hemp and on its medical use counterpart. The phyto-complex of cannabis plants is made up of more than 500 different constituents, of which one hundred or more belong to the cannabinoid class [47]. Therefore, the applied extraction technique can exert considerable influence on the active molecules administered to patients.

The possibility that the co-presence of numerous molecules, cannabinoids and others, in preparations obtained from cannabis and administered to patients may lead to a greater effect than the sum of its contributing parts has been called the "entourage effect". This effect was first hypothesised by Mechoulam and Ben-Shabat [48]. Synergy between cannabinoids and between cannabinoids and terpenes in cannabis has been investigated. Although there are many promising studies on this topic, the incomplete understanding of the mechanism behind the entourage effect in cannabis preparations makes it difficult to follow regular drug development and approval procedures [49-51]. Recently, the role of the entourage effect of cannabis-based products in pain has been evaluated. Although definitive studies are still required, there is increasing evidence to support the beneficial effects of plant-derived cannabinoids in providing pain relief [52].

Given the widespread interest in the entourage effect, several studies have been performed on complex cannabis extracts, often called the "full-extracts". Recently, in order to investigate the effect of cannabis extracts on opioid use and HRQoL in chronic pain patients, soft gels containing $\mathrm{CBD}$, THC, CBDV, CBDA, CBC and a terpene blend were administered in a single-arm cohort study. The results suggest that the administered extract allowed patients to significantly reduce opioid use, while also providing improvements in their HRQoL [53]. The role of cannabis complex extracts in pain, particularly of the neuropathic type, has also been reviewed, and it was highlighted how several clinical studies using cannabis extracts have provided promising results. On the other hand, some clinical trials have highlighted the reduced beneficial effects of purified THC and/or CBD. This, according to the authors, underlines the possibility that studies using a purified THC or CBD may not truly reflect the extracts' benefits [54].

Regarding the extraction methods, the most widely used solvent for plant solid-liquid extraction is ethanol, because of its cannabinoid high extraction power; other solvents are 
generally used for analytical purposes [55, 56]. Ethanol has also been reported in the German pharmacopoeia Cannabis flos monograph, which describes dynamic maceration as the method of choice for cannabinoid extraction [55, 57].

Alternative types of extraction, such as ultrasoundassisted extraction, microwave-assisted extraction and supercritical fluid extraction have also been described $[55,56$, 58-60]. All these extraction methods are difficult to apply to medical use, because of the costs and the difficulty of their preparation in the labs of hospitals and community pharmacies, which are usually tasked with preparing cannabis for medical purposes [61]. Supercritical $\mathrm{CO}_{2}$ extraction is, however, taken into account by the German pharmacopoeia Cannabis extractun normatum monograph as the preferable method [62, 63].

On the other hand, water has been reported to be a bad solvent for cannabinoids, raising the relevant issue of waterbased preparations derived from cannabis, such as decoction [56, 64-66]. Prescribing a cannabis-based decoction, considering the low recovery rates of THC and CBD and, consequently, the high volume of preparation that the patient would have to ingest, should not be the first choice for cannabis-based therapies. More important, only the soluble cannabinoids acids can be extracted in water, and these will not significantly decarboxylated successively

[67]. Furthermore, the high raw material costs necessary to obtain the desired doses of active molecules should be seriously taken into account [46].

Cannabis-oil preparation has aroused a lot of interest in recent years for two main reasons: its ability to easily modulate the dose during therapy, and the better bioavailability of the active components, due to the lipophilicity of the formulation [68]. These preparations generally involve the use of European Pharmacopoeia grade olive oil, which can positively affect the digestibility of the finished product as lipid sources, rich in saturated fatty acids, are less sensitive to oxidation phenomena after hot maceration, resulting in minimal deterioration during storage [69-71].

In this context, although there are no official shared protocols, cannabis-based formulations have been prepared on prescription by pharmacists in recent years [61]. The methods for preparing cannabis oils for medical purposes that have been developed since 2018 have been summarised in Table 1, and some differences have been highlighted. First of all, the weight-to-volume ratio between plant material and oil can vary from 0.02 to $0.2 \mathrm{~g} / \mathrm{mL}$ of oil. Furthermore, pre-treatment can be applied to the plant material; it is a relevant step and may consist of crushing the inflorescences in order to reduce their particle size, as well as heating the plant material in order to decarboxylate THCA and CBDA into the corresponding neutral forms (THC and CBD). The decarboxylation process has been recommended as it turns the acidic cannabinoids into their neutral counterparts THC and $\mathrm{CBD}$, which can be more easily absorbed through the intestine [43]. However, some authors have pointed out the therapeutic value of acidic cannabinoids [43, 44].

Moreover, different inflorescence maceration times and applied temperatures have been applied.

Cannabis oils are usually characterised in terms of their THC and/or CBD content. Considering the potential role of the "entourage" effect, some extraction methods have been developed to preserve the terpenes naturally present in cannabis, although there is not yet sufficient evidence for their role in therapy [72]. Low temperatures (maximum $115^{\circ} \mathrm{C}$ ) have been applied for this purpose. Low-temperature treatment can also be associated with ethanol co-extraction and microwaves [72].

The feasibility of using medium-chain triglycerides has also been recently investigated and the results have been compared with data obtained from olive oil preparations, with excellent results being reported for the quantity and stability of the extracted terpenes. However, of course, the cannabinoid profile may be affected by the use of these different lipids [70].

Regardless of the interest in terpenes, the effect of adding surfactants to water or ethanol has also been investigated, as has the use of ultrasound and microwaves, to improve extraction in oil or ethanol [73].

A great variability in the concentration of active molecules in the oils has generally been found and this depends on the use of different varieties of cannabis and, of course, on their possible natural fluctuation in the inflorescences $[74,75]$. However, the principal variability derives from the application of different preparation protocols in pharmacies $[75,76]$. The variability in oil composition means that titration is fundamental to prescribing the correct dosage. Moreover, it is necessary in order to clarify what molecules, in addition to THC and CBD, are to be considered during titration $[75,77]$. The administration of formulations that contain known quantities of active molecules is essential to ensure the uniformity of therapies, which can lead to the consequent optimisation of the risks/benefits [78]. Furthermore, the need to know the exact composition of the preparations is linked to the possibility of evaluating the effectiveness of the entourage effect.

In order to respond to the needs of quality control and quality standards in herbal products and extracts, a Cannabis Expert Panel was convened by USP in 2016 at the direction of the USP Botanical Dietary Supplements and Herbal Medicines Expert Committee. A very important review that was recently produced by the Expert Panel clearly describes the scientific quality attributes and related standards of cannabis [79].

One aspect that is not to be overlooked is the fact that the organoleptic characteristics of the oils usually are particularly unpleasant. In order to facilitate medical cannabis 
Table 1 Galenic preparations

\begin{tabular}{|c|c|c|c|c|c|c|c|}
\hline Plant material & $\begin{array}{l}\text { Cannabis/ } \\
\text { olive oil ratio }\end{array}$ & $\begin{array}{l}\text { Cannabis treatment } \\
\text { to reduce particle } \\
\text { size }\end{array}$ & $\begin{array}{l}\text { Cannabis treatments } \\
\text { before oil extraction }\end{array}$ & $\begin{array}{l}\text { Olive oil extraction } \\
\text { conditions }\end{array}$ & Number of samples & Stability tests & References \\
\hline Bedrocan $^{\circledR}$ & $1: 10$ & Yes & $\begin{array}{l}115^{\circ} \mathrm{C}, 40 \mathrm{~min} \\
145^{\circ} \mathrm{C}, 30 \mathrm{~min} \\
\mathrm{RT}\end{array}$ & $\begin{array}{l}70{ }^{\circ} \mathrm{C}, 40 \mathrm{~min} \\
100^{\circ} \mathrm{C}, 40 \mathrm{~min} \\
100^{\circ} \mathrm{C}, 120 \mathrm{~min} \\
110^{\circ} \mathrm{C}, 120 \mathrm{~min}\end{array}$ & $\begin{array}{l}6 \text { samples in the } \\
\text { optimised condi- } \\
\text { tions }\end{array}$ & $\begin{array}{l}\text { Refrigerator, } 3 \\
\text { weeks }\end{array}$ & [61] \\
\hline Bedrocan $^{\circledR} ;$ Bediol $^{\circledR}$ & $1: 10$ & Yes & $145^{\circ} \mathrm{C}, 30 \mathrm{~min}$ & $\begin{array}{l}\text { Ultrasound extrac- } \\
\text { tion ( } 35 \mathrm{KHz} 30 \\
\text { min) at RT }\end{array}$ & Not declared & $\begin{array}{l}\text { Refrigerator or RT, } \\
6 \text { weeks }\end{array}$ & [71] \\
\hline Bedrocan $^{\circledR}$ & $1: 10$ & Yes & $\begin{array}{l}\text { A: } 80 \% \text { of the inflo- } \\
\text { rescences: } \\
\text { 1. } 115^{\circ} \mathrm{C}, 90 \text { min } \\
\text { 2. Addition of olive } \\
\text { oil } \\
\text { B: } 20 \% \text { of the inflo- } \\
\text { rescences: } \\
\text { 3. Ethanolic extrac- } \\
\text { tion } \\
\text { 4. Ethanol evapora- } \\
\text { tion } \\
\text { 5. Heating step: } 115 \\
{ }^{\circ} \mathrm{C}, 30 \text { min } \\
\text { 6. Addition of olive } \\
\text { oil }\end{array}$ & $\begin{array}{l}\text { Mixture of A and } \\
\text { B: maceration } \\
\text { under mechanical } \\
\text { stirring for } 24 \mathrm{~h} \text { at } \\
\text { room temperature }\end{array}$ & 2 & No & [72] \\
\hline Bedrocan $^{\circledR}$ & $1: 10$ & Yes & $\begin{array}{l}\text { 1. Steam distilla- } \\
\text { tion: } 100^{\circ} \mathrm{C}, 120 \\
\text { min to obtain the } \\
\text { essential oil } \\
\text { 2. Removal of } \\
\text { residual water } \\
\text { from the plant } \\
\text { material } \\
\text { 3. Addition of } \\
\text { olive oil to plant } \\
\text { material }\end{array}$ & $\begin{array}{l}\text { 1. Olive extract } \\
\text { obtained at } 98^{\circ} \mathrm{C} \text {, } \\
60 \text { min } \\
\text { 2. Addition of the } \\
\text { essential oil to the } \\
\text { olive oil extract }\end{array}$ & 2 & No & [72] \\
\hline Bedrocan $^{\circledR}$ & $1: 10$ & Yes & $\begin{array}{l}\text { (A) Microwave } \\
\text { hydro-distillation } \\
\text { to obtain the } \\
\text { essential oil: } 500 \\
\mathrm{~W} \text { for } 3 \text { min, then } \\
320 \mathrm{~W} \text { for } 10 \mathrm{~min} \\
\text { and, finally, } 290 \\
\mathrm{~W} \text { for } 60 \mathrm{~min} \\
\text { (B) Removal of } \\
\text { residual water } \\
\text { from the plant } \\
\text { material } \\
\text { (C) Addition of } \\
\text { olive oil to plant } \\
\text { material }\end{array}$ & $\begin{array}{l}\text { 1. Olive extract } \\
\text { obtained at } 98^{\circ} \mathrm{C} \\
\text { for } 60 \mathrm{~min} \\
\text { 2. Addition of the } \\
\text { essential oil to the } \\
\text { olive oil extract }\end{array}$ & 2 & No & [72] \\
\hline FM2 & $1: 10 ; 1: 5$ & Yes & $\begin{array}{l}115^{\circ} \mathrm{C} \text { for } 40 \mathrm{~min} \\
140^{\circ} \mathrm{C} \text { for } 30 \mathrm{~min} \\
\text { Room temperature }\end{array}$ & $\begin{array}{l}100^{\circ} \mathrm{C} \text { for } 30,60, \\
120 \mathrm{~min}\end{array}$ & $>100$ & $\begin{array}{l}\text { Refrigerator or RT, } \\
6 \text { months }\end{array}$ & {$[46,78]$} \\
\hline Bedrocan $^{\circledR}$, Bediol $^{\circledR}$ & $1: 10$ & Yes & $\begin{array}{l}100^{\circ} \mathrm{C}, 30 \mathrm{~min} \\
125^{\circ} \mathrm{C}, 30 \mathrm{~min}\end{array}$ & $100^{\circ} \mathrm{C}$ for $30 \mathrm{~min}$ & 3 & $4{ }^{\circ} \mathrm{C}, 3$ months & [70] \\
\hline $\begin{array}{c}\text { Bediol }^{\circledR}, \mathrm{FM}^{\circledR}, \\
\text { Bedrocan }^{\circledR}\end{array}$ & $1: 50$ & Yes & $120-130{ }^{\circ} \mathrm{C}, 30 \mathrm{~min}$ & $\begin{array}{l}\text { Microwave assisted } \\
\text { oil extraction: } 90 \\
{ }^{\circ} \mathrm{C}, 3 \text { min }\end{array}$ & Not declared & No & [73] \\
\hline
\end{tabular}

Bediol $^{\circledR}: 6.3$ THC, $8 \%$ CBD; $^{\text {Bedrocan }}{ }^{\circledR}: 22 \%$ THC; < 1\% CBD; FM2: 5-8\% THC, $7.5-12 \%$ CBD

$R T$ room temperature 
consumption by patients, a standard procedure has recently been developed for the preparation of hard capsules with cannabis oil. Masking the organoleptic characteristics of the oil may also have the advantage of allowing the presence of a placebo group in a clinical trial. Capsules have been selected because this pharmaceutical form can be easily prepared in a reproducible way in a pharmacy [78].

Apart from the literature data on the performance of the different applied extraction techniques, the efficacy of the magistral formulas also needs to be clinically investigated, not only in terms of pain treatment, but also in consideration of the fact that prescriptions are usually added to standard treatments. There are currently no significant data on the clinical outcomes of these preparations, in part, because data on the efficacy of the administered preparations are usually not reported by the literature, although guidelines are available for prescribing or preparing magistral formulas. This is also true because, in some cases, preparations, such as decoctions, are prepared at home by patients, making them less standardisable and outcomes difficult to evaluate. Consequently, the decoction is performed in different ways. According to the most known information, provided by the Dutch Office for Medicinal Cannabis to patients, decoctions (called "teas") could be prepared as follows: bring the water to a boil and add $1 \mathrm{~g} / \mathrm{L}$ of cannabis. Lower the heat, cover with a lid and allow to simmer gently for 15 minutes then remove from heat and filter [80].

Nevertheless, cannabis preparations are usually prescribed for the treatment of several conditions, such as pain, cancer, epilepsy, insomnia, depression and anxiety, attention deficit/hyperactivity disorder (ADHD), autism, cluster headaches and Crohn's disease, irritable bowel syndrome, restless legs syndrome and Tourette's syndrome [44].

The idea of using capsules has also been explored in a recent trial in which purified extracts of cannabinoids, titrated in THC and CBD, have been added to blends of coconut oil, monoglyceride and diglyceride and combined with carrageenan to obtain a lipid-based delivery system. The aim of the study was to evaluate the effect of dosagecontrolled cannabis capsules in cancer-related cachexia and anorexia syndrome in advanced cancer patients. Despite various limitations, this preliminary study demonstrated weight increases in some patients, highlighting the need for a larger study [81].

\section{Pharmaceutical-Grade Cannabinoid Formulations}

\subsection{Oral Administration}

The oral administration route of cannabinoids is favoured over the other routes because it offers several advantages including safety, good patient compliance, ease of ingestion, pain avoidance, and the versatility to accommodate various types of drugs. Furthermore, exposure time is longer, and the side effects are significantly less intense.

Cannabinoids, indeed, have poor oral bioavailability (estimated to be as low as $6 \%$ of a similar dose administered intravenously). Pure THC and CBD show similar plasma concentration-time profiles, with a delay of $120 \mathrm{~min}$ before reaching peak concentration [42]. Bioavailability increases when it is co-administered with food lipids. A cannabis formulation in sesame oil (a vehicle with long chain triglycerides [LCT], containing $42 \%$ oleic, $40 \%$ linoleic and $16 \%$ palmitic acids) significantly improved the bioavailability of cannabinoids [82].

The contribution of intestinal lymphatic transport is also worthy of note in the case of highly lipophilic compounds [83]. Indeed, the fatty acids generated by the lipolysis of medium and long chain triglycerides undergo several different processes: long chain fatty acids are associated to chylomicrons that are secreted into the lymphatic system instead of the portal vein. Thus, THC and CBD, used in combination with LCT, can bypass the liver, decreasing potential presystemic metabolism [41]. By avoiding loss in pre-systemic metabolism in the liver, cannabinoids can reach $22 \%$ bioavailability [84].

The role of high-fat meals on pharmacokinetic behaviour is also relevant. A formulation for CBD (administered as a single $1500 \mathrm{mg}$ dose at $100 \mathrm{mg} / \mathrm{mL}$ in a sesame-oil vehicle) showed an absolute oral bioavailability of about $6 \%$ after intake in the fasting state, while co-administration with a high-fat meal would be expected to result in a bioavailability of about $25 \%$ and in reduced inter- and intra-subject variability, compared with the fasting state [40]. Moreover, THC showed similar bioavailability behaviour when administered with sesame oil [43].

Furthermore, the potential role of cannabinoid metabolites, especially active metabolites such as 7-hydroxy-CBD, should be considered, as outlined in recent studies in subjects with renal or hepatic impairments [85-87].

Oils that are formulated into capsule pellets and solid tablets currently allow pharmaceutical-grade compounds to be conveniently and accurately dosed.

Two important considerations to make when evaluating cannabinoid formulations are the pharmacokinetic goal and intended use. For example, if the indication is to treat acute pain, then a faster $T_{\max }$ and higher $C_{\max }$ may be desirable, while also potentially helping to decrease the risk of overdose via premature repeat self-administration. Alternatively, a larger AUC may be preferable for use as a chronic treatment if a user follows a regular dosing schedule.

Nevertheless, an ideal oral dosage form that has consistent delivery and high bioavailability is not yet available and is therefore warranted. Many companies have invested 
huge amounts of effort into improving pharmaceutical oral dosage. The different strategies taken have been detailed below, and have been marketed. The most advanced formulations have been summarised in Table 2. Table 3 summarises the ongoing clinical trials on pain treatments. Only the most recent reports on active research and the latest development phases are described. To complete the information and allow the reader to move more deeply into the composition and technology involved in the formulation procedures, the most significant data from related patents are also reported.

Further improvements can be expected with the use of nanotechnology sciences as summarised in Sect. 6.1.1.

Two pharmaceutical preparations of purified THC are currently available. Dronabinol is the semi-synthetic form of THC, which is available in the capsule form Marinol ${ }^{\circledR}$ (Abbvie Inc. US) and as a solution Syndros ${ }^{\circledR}$, which was originally produced by Insys Therapeutics US and is now marketed by Benuvia Therapeutics, USA.

Marinol ${ }^{\circledR}$ is supplied as round, soft gelatin capsules that either contain $2.5,5$ or $10 \mathrm{mg}$ of dronabinol formulated in sesame oil.

A Syndros ${ }^{\circledR}$ solution contains $5 \mathrm{mg}$ of dronabinol in $1 \mathrm{~mL}$ of the inactive ingredients: $50 \%(\mathrm{w} / \mathrm{w}$ ) dehydrated alcohol, polyethylene glycol 400, propylene glycol, plus preservatives and antioxidants.

Marinol ${ }^{\circledR}$ and Syndros ${ }^{\circledR}$ were approved by the FDA for the treatment of anorexia associated with weight loss in patients with AIDS. It has also been approved for the nausea and vomiting associated with cancer chemotherapy in patients who have failed to respond adequately to conventional antiemetic treatments (NDA 18-651/S-021).

In Europe, dronabinol and CBD received an initial evaluation as orphan drugs for the treatment of glioma in 2015, but this was later withdrawn. Medicinal products that contain dronabinol are available in few EU countries (for compassionate grounds and for magistral preparations). Two ongoing studies appear in the EU Clinical Trials Register: one for patients with pancreatic cancer in the palliative state (2017-000530-54), and the second on the efficacy and safety of THC in behavioural disturbances and pain in dementia (2011-005289-39).

In Europe, a liquid formulation of dronabinol (named BX 1), produced by Bionorica SE (D), began a Phase III trial to evaluate the efficacy and safety as adjuvant therapy to first-line standard chemotherapy in patients with metastatic pancreatic cancer $(n=140)$ for the improvement of chemotherapy- and tumour-related symptoms (EudraCT2019-000616-28; NCT03984214). A Phase III trial was set up to evaluate the safety and efficacy of dronabinol in patients $(n=548)$ with spasticity due to multiple sclerosis (EudraCT2018-000001-23; NCT03756974). More recently, in June 2020, a Phase II trial for post-traumatic stress disorders was devised in collaboration with Charité University (Berlin, D) (NCT04448808).

A low-dose oral formulation of dronabinol is being developed by RespireRx Pharmaceuticals (USA), in collaboration with the University of Illinois and Northwestern University, for the treatment of obstructive sleep apnoea (OSA). This therapy was previously being developed by Pier Pharmaceuticals (USA) as a pellet formulation that combined dronabinol and sodium lauryl sulfate (1:1) plus inert excipients. The drug is administered orally as an instant-release formulation, 60 minutes before bedtime. Clinical development is underway in the USA. Although no clinical trials have been reported in Letendre's patent, a dose of $2.5 \mathrm{mg}$ of THC was claimed to provide a significant therapeutic effect (reduction of sleep apnoea) [88].

Namisol $^{\mathrm{TM}}$ is the name of an oral tablet formulation of dronabinol that is developed by Echo Pharmaceuticals b.v., (NL). This was produced using Alitra ${ }^{\mathrm{TM}}$, an emulsifying drug delivery technology. This technology involved the preparation of THC microgranules (size 30 micron) with the surfactant sucrose monolaurate (about $7 \% \mathrm{w} / \mathrm{w}$ ), to enhance solubility, and then tableting with other excipients [89]. Klumpers et al. have reported pharmacokinetic/pharmacodynamic evaluations, indicating that the pharmacokinetic differences after oral and sublingual (crushed tablets) administration were small [90]. Nevertheless, this formulation showed a short time to reach maximal THC concentration (39-56 $\mathrm{min}$ ) in comparison to other cannabinoid formulations [91]. Safety and tolerability were also reported, as well the pharmacodynamics $(n=12)$ [92]. Interestingly, the effects of THC were smaller than expected for older people. A clinical trial on postoperative pain concluded in 2014, and this formulation was recently planned for study in a Phase II trial for anorexia in Australia (ACTRN12619000491167).

Nabilone (Cesamet ${ }^{\mathrm{TM}}$ from Bausch Health Companies) is an orally active synthetic cannabinoid derivative that differs structurally from THC as its C-ring is saturated and contains a C-9 ketone group (Fig. 2). Nabilone is available, with polyvinylpyrrolidone and starch excipients, as a capsule $(1 \mathrm{mg}$ of drug) and has been marketed in the USA since 2006 for the treatment of chemotherapy-induced nausea and vomiting in patients who have not responded to conventional antiemetic treatments [93].

Cyclodextrins, from a pharmaceutical point of view, are importantly used for enhancing the aqueous solubility of poorly soluble drugs, improving stability and masking the unpleasant taste/odour of a formulation [94]. A fastdisintegrating, controlled-release formulation of nabilone (Canemes ${ }^{\circledR}$ ) is being developed by AOP Orphan Pharmaceuticals (AT) for the treatment of spasticity-associated pain and non-motor symptoms associated with Parkinson's disease [95]. AOP Orphan Pharmaceuticals is the proprietor of a formulation in which randomly methylated 


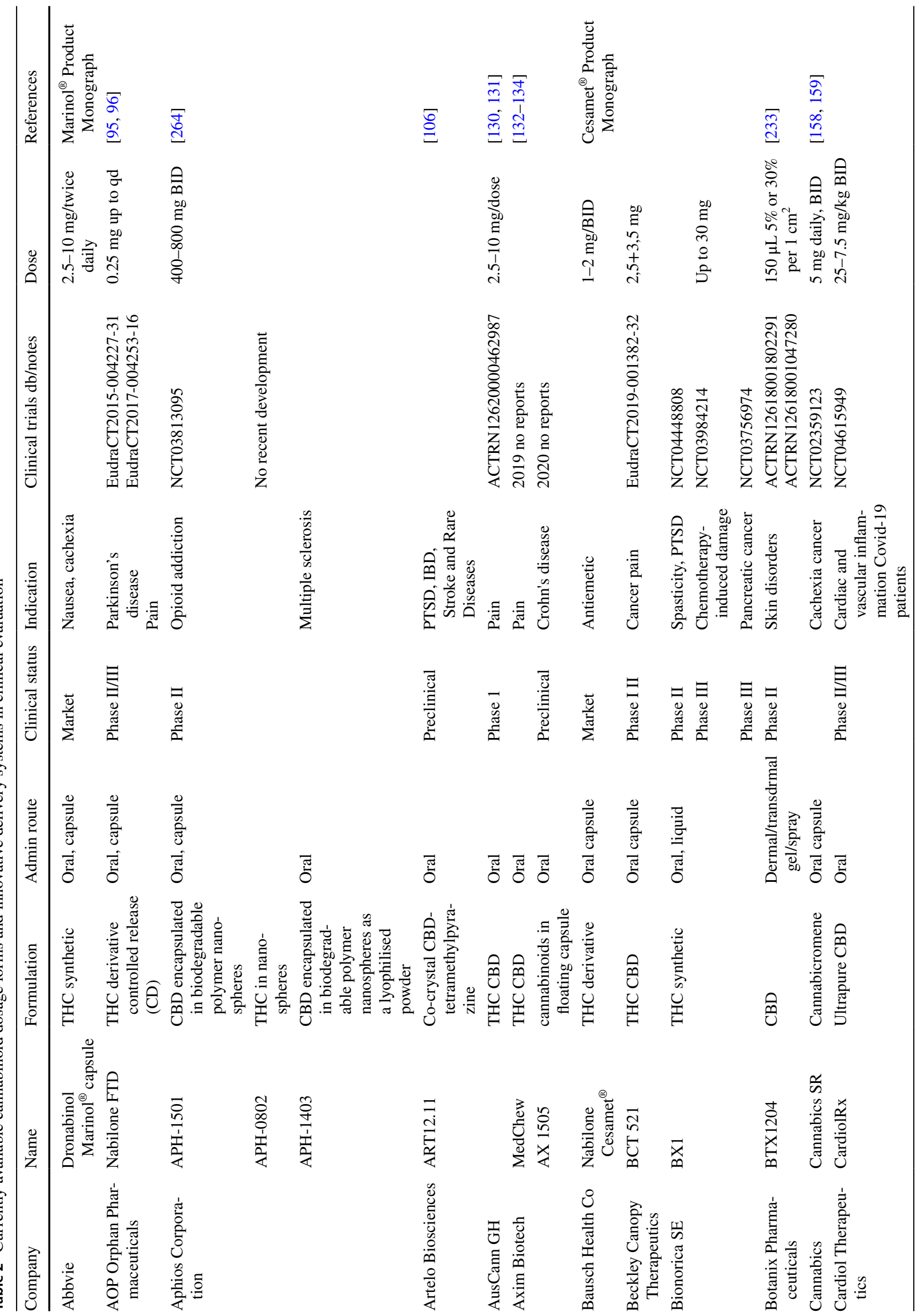




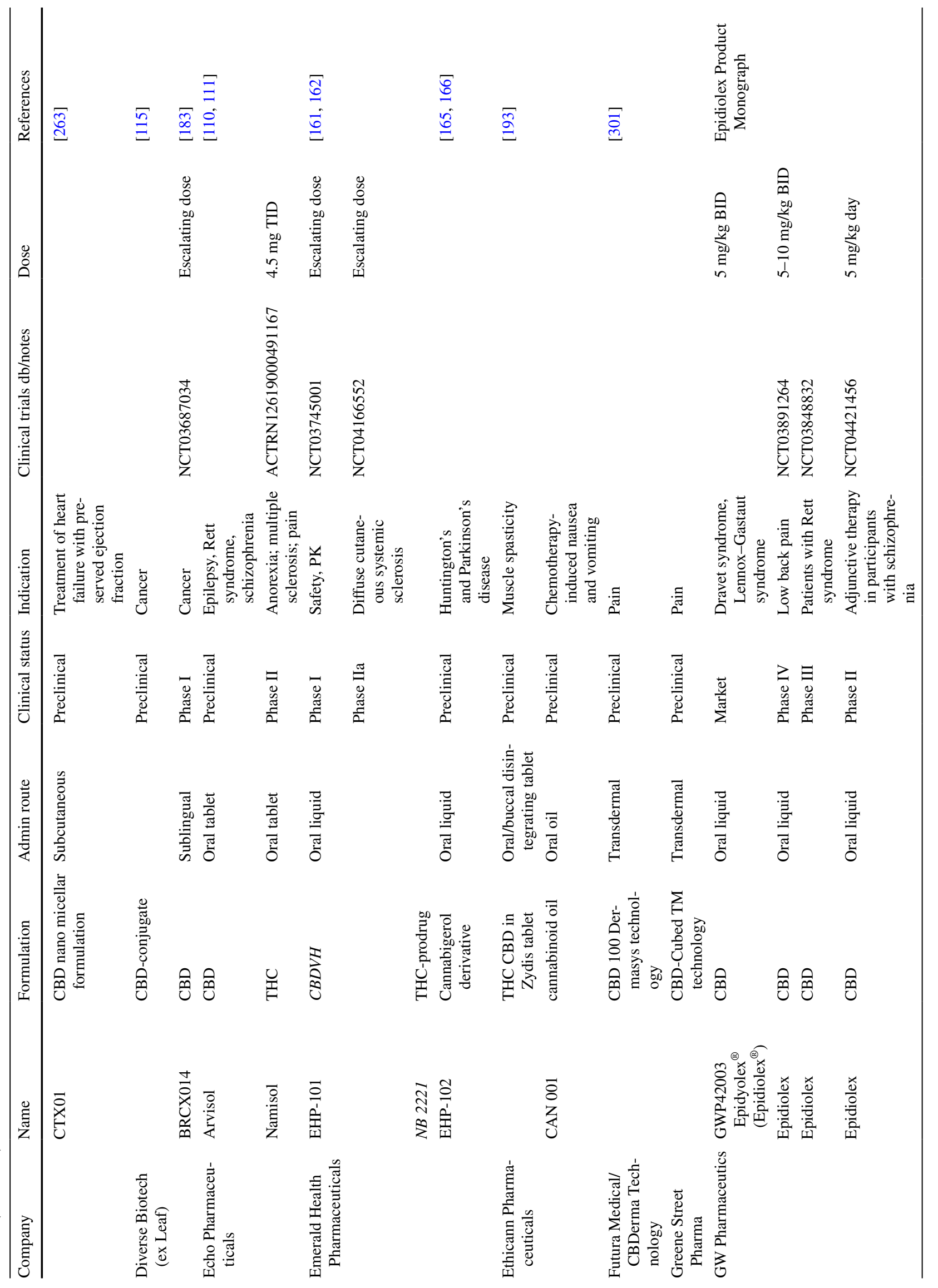




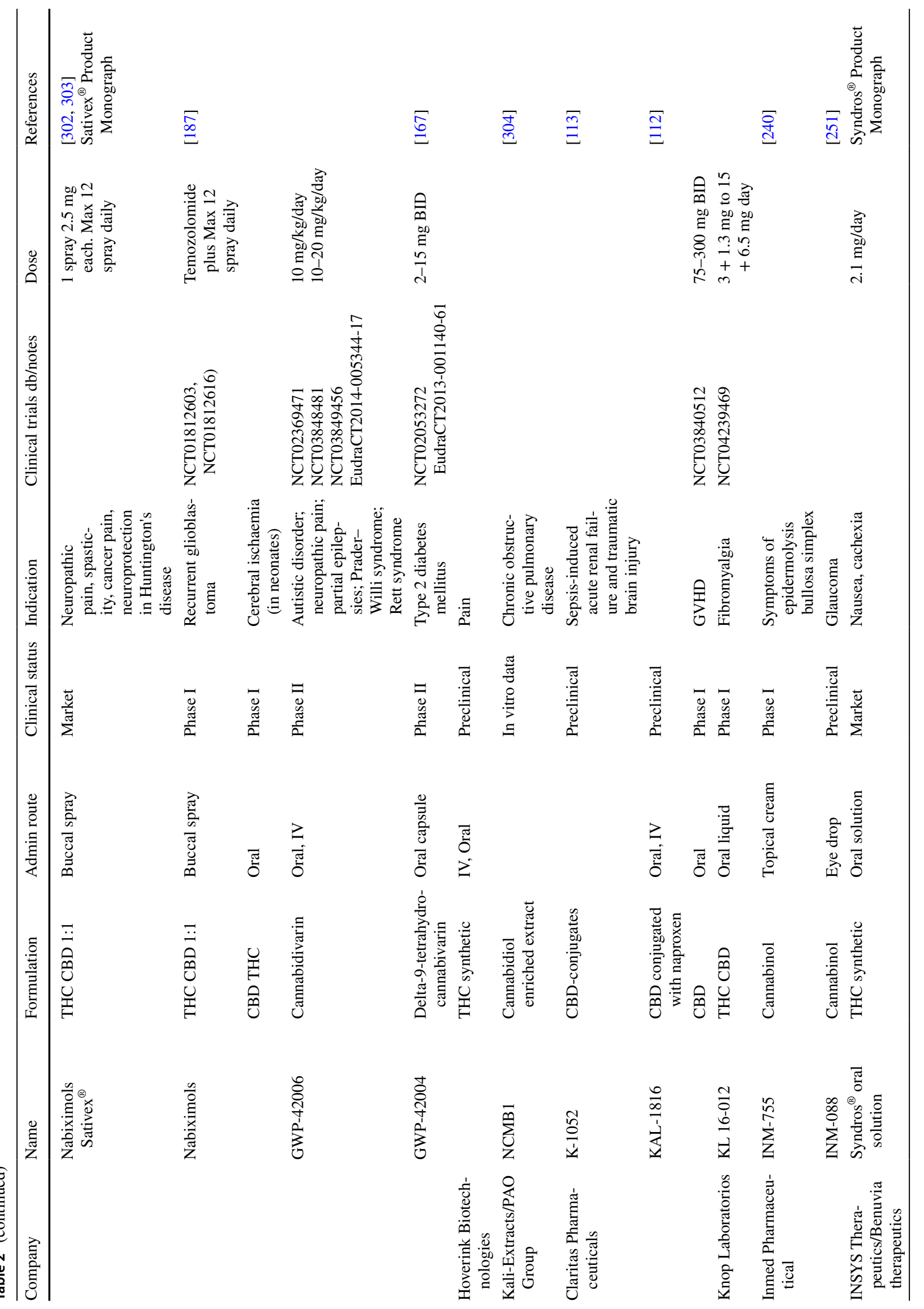




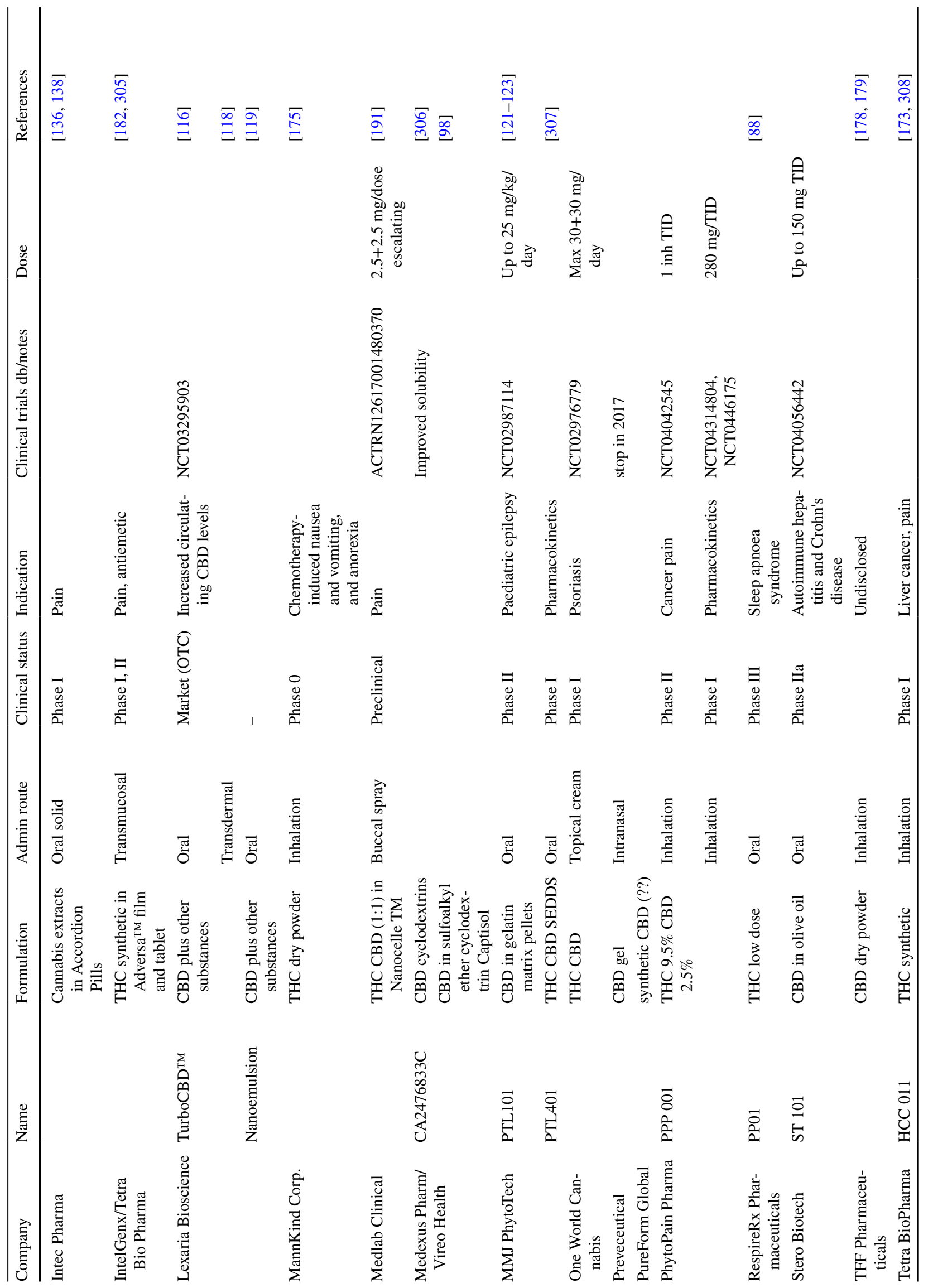




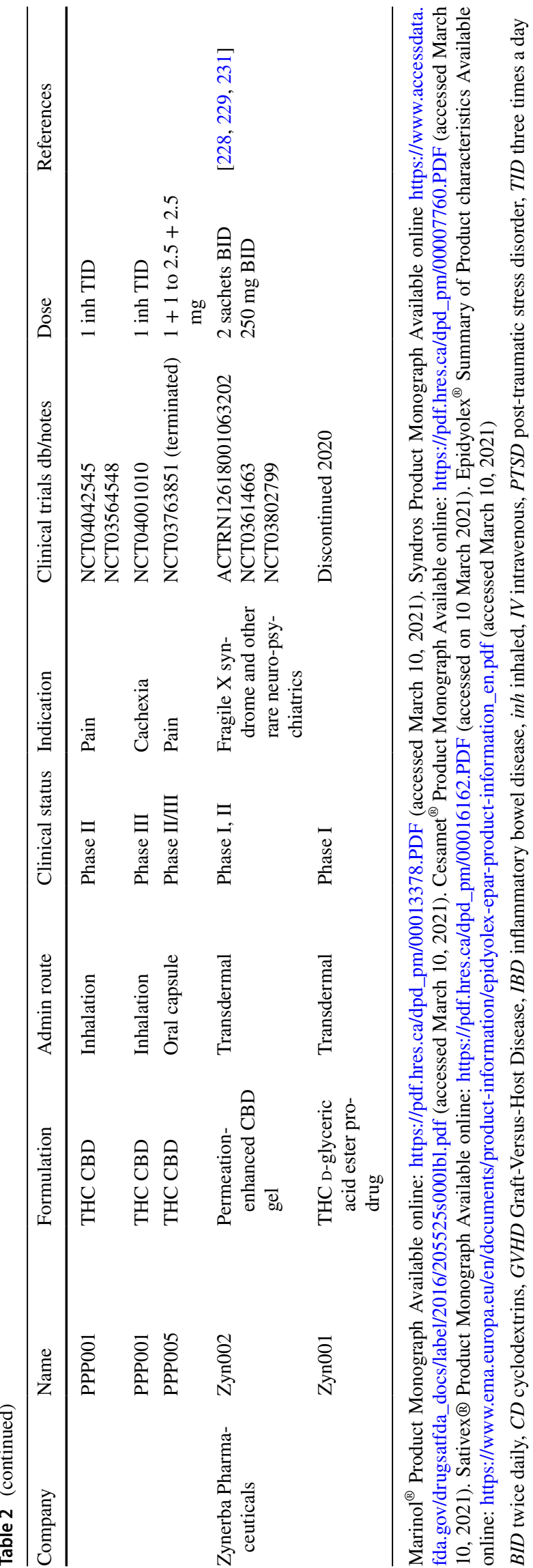

$\beta$-cyclodextrin ( $\beta$-CD) inclusion complexes of nabilone significantly increased the aqueous solubility and bioavailability of the drug. Of the tested CDs, randomly methylated $\beta-C D$ was found to be preferable [96]. A pilot Phase II trial of Canemes ${ }^{\circledR}$ for pain was initiated in 2016 in Austria (EudraCT2015-004227-31), while Phase III development is underway in Austria for Parkinson's disease in the elderly (EudraCT2017-004253-16).

It is important to note that the chemical derivatisation of CDs may improve their water solubility (2-hydroxypropyl$\beta$-CD has aqueous solubilities of over $500 \mathrm{mg} / \mathrm{mL}$ ) and can also modulate the binding affinities of the hosts for guest molecules that bind to the hydrophobic cavity of CDs [97]. A sulfoalkyl ether derivative further improves their aqueous solubility. In a patent from Vireo Health LLC (USA), the water solubility of cannabinoids was enhanced by complexation with sulfoalkyl- $\beta-C D$, and again further by the presence of Cremophor EL (polyoxyl-35 castor oil) [98].

Now moving to CBD-based products, the best-known marketed medicine is Epidiolex ${ }^{\circledR}$ (from GW Pharmaceuticals plc, UK). It is a liquid pharmaceutical-grade formulation of purified CBD containing $100 \mathrm{mg} / \mathrm{mL}$ drug dissolved in sesame oil and $10 \%$ ethanol. Epidiolex ${ }^{\circledR}$ has been approved in the USA and Europe as an adjuvant treatment in Dravet syndrome, Lennox-Gastaut syndrome and severe myoclonic epilepsy in infancy (aged 2 years and above). Results from double-blind, placebo-controlled trials have recently been published [99-101]. The same formulation was also approved by the EMA in 2019 (with name Epidyolex) for the same indications, except that regulatory approval in Europe is limited to patients receiving concomitant therapy with clobazam [10].

As reported in the summary of product characteristics, the role of patient's diet is relevant. Indeed, taking CBD with alcohol caused enhanced exposure to CBD, with a $63 \%$ greater AUC. Moreover, the co-administration of CBD ( 750 or $1500 \mathrm{mg}$ ) with a high-fat/high-calorie meal increased the rate and extent of absorption (5-fold increase in $C_{\max }$ and 4-fold increase in AUC).

Clinical development is underway in a number of countries for the treatment of several diseases/symptoms: cerebral ischaemia, epilepsy, infantile spasms, opioid abuse, heroin-related disorders, Parkinson's disease, Rett syndrome, schizophrenia, and tuberous sclerosis. Preclinical development is also underway for Duchenne muscular dystrophy and ovarian cancer in the UK.

GW Pharmaceutics is currently enrolling patients (NCT03891264) in Phase IV research to evaluate the presence of neuroinflammation in the brains and spinal cords of patients with low back pain, and whether CBD effects levels of neuroinflammation. Further clinical trials involving the oral administration of CBD in pain treatment 
Table 3 Ongoing clinical trials of cannabinoids and pain in detail

\begin{tabular}{|c|c|c|c|c|c|}
\hline Clinical trial identifier & Study title & Sponsor & Active substance & Formulation/route & $\begin{array}{l}\text { Phase (estimate com- } \\
\text { pletion) }\end{array}$ \\
\hline NCT02460692 & $\begin{array}{l}\text { Dronabinol and } \\
\text { vaporised cannabis } \\
\text { in neuropathic low } \\
\text { back pain }\end{array}$ & $\begin{array}{l}\text { University of Cali- } \\
\text { fornia, San Diego }\end{array}$ & $\begin{array}{c}\text { THC or vaporised } \\
\text { cannabis } 3.7 \% \\
\text { THC/5.6\% CBD }\end{array}$ & Inhalation & II (2020) \\
\hline NCT04298528 & $\begin{array}{l}\text { THC post-operative } \\
\text { pain in total joint } \\
\text { arthroplasty }\end{array}$ & $\begin{array}{c}\text { Colorado joint } \\
\text { replacement }\end{array}$ & THC, dronabinol & Oral & III (2024) \\
\hline NCT03978156 & $\begin{array}{l}\text { THC pain and } \\
\text { inflammation in } \\
\text { adults living with } \\
\text { sickle cell disease }\end{array}$ & Yale University & THC, dronabinol & Oral & $\mathrm{I}(2021)^{\mathrm{a}}$ \\
\hline NCT03099005 & $\begin{array}{l}\text { Effect of cannabis } \\
\text { and endocan- } \\
\text { nabinoids on HIV } \\
\text { Neuropathic pain }\end{array}$ & $\begin{array}{l}\text { University of Cali- } \\
\text { fornia, San Diego }\end{array}$ & $\begin{array}{r}\text { Cannabis (different } \\
\text { doses CBD THC) }\end{array}$ & $\begin{array}{l}\text { Vaporisation of can- } \\
\text { nabis }\end{array}$ & II (2021) \\
\hline NCT03984214 & $\begin{array}{l}\text { Improvement of } \\
\text { chemotherapy- } \\
\text { induced and tumor- } \\
\text { related symptoms } \\
\text { in advanced } \\
\text { pancreatic cancer }\end{array}$ & Bionorica SE & THC dronabinol & Oral & III (2022) \\
\hline NCT04448808 & $\begin{array}{l}\text { Nightmares in post- } \\
\text { traumatic stress } \\
\text { disorder }\end{array}$ & Bionorica SE & THC & Oral & II (2022) \\
\hline NCT03564548 & $\begin{array}{l}\text { Cannabinoids versus } \\
\text { immediate-release } \\
\text { oral opioids for } \\
\text { the management } \\
\text { of breakthrough } \\
\text { cancer pain }\end{array}$ & Tetra Bio-Pharma & THC (PPP011) & Inhalation & II (2022) \\
\hline NCT03763851 & $\begin{array}{l}\text { THC CBD and radia- } \\
\text { tion therapy for } \\
\text { the management } \\
\text { of pain }\end{array}$ & Tetra Bio-Pharma & THC CBD (PPP005) & Oral & $\begin{array}{l}\text { II (2018) Terminated } \\
\text { (due to mycotoxin } \\
\text { potential contami- } \\
\text { nation of one lot of } \\
\text { study drug) }\end{array}$ \\
\hline $\begin{array}{l}\text { EudraCT2015-004227-31 } \\
\text { EudraCT2017-004253-16 }\end{array}$ & $\begin{array}{l}\text { Parkinson's disease/ } \\
\text { pain }\end{array}$ & $\begin{array}{l}\text { AOP Orphan Phar- } \\
\text { maceuticals }\end{array}$ & Nabilone FTD & & \\
\hline NCT03891264 & $\begin{array}{l}\text { Reduction of neuro- } \\
\text { inflammation in } \\
\text { chronic back pain }\end{array}$ & $\begin{array}{l}\text { GW Pharmaceutics } \\
\text { Massachusetts } \\
\text { General Hospital }\end{array}$ & CBD & Oral & IV (2021) \\
\hline NCT04239469 & $\begin{array}{l}\text { Fibromyalgia refrac- } \\
\text { tory to conven- } \\
\text { tional treatment }\end{array}$ & Knop Laboratorios & THC CBD & Oral & II (2020) \\
\hline NCT02359123 & $\begin{array}{l}\text { Improve quality of } \\
\text { life in advanced } \\
\text { cancers }\end{array}$ & $\begin{array}{l}\text { Cannabics Pharma- } \\
\text { ceuticals Inc. }\end{array}$ & Cannabis oil & Oral & n.a. (2018) \\
\hline NCT04195269 & $\begin{array}{l}\text { Osteoarthritis of the } \\
\text { knee pain }\end{array}$ & Pure Green & THC CBD & Sublingual & II (2020) \\
\hline NCT04091789 & $\begin{array}{l}\text { Treatment of dys- } \\
\text { menorrhea }\end{array}$ & Pure Green & THC CBD & Sublingual & II (2020) \\
\hline NCT04527003 & Endometriosis pain & $\begin{array}{l}\text { Milton S. Hershey } \\
\text { Medical Center }\end{array}$ & $\mathrm{CBD}$ & Sublingual & III (2022) \\
\hline NCT04001010 & $\begin{array}{l}\text { Cachexia progres- } \\
\text { sion/pain }\end{array}$ & Tetra Bio-Pharma & THC CBD & Inhalation & III (2021) \\
\hline
\end{tabular}


Table 3 (continued)

\begin{tabular}{|c|c|c|c|c|c|}
\hline Clinical trial identifier & Study title & Sponsor & Active substance & Formulation/route & $\begin{array}{l}\text { Phase (estimate com- } \\
\text { pletion) }\end{array}$ \\
\hline EUdraCT2017-000530-54 & $\begin{array}{l}\text { Cannabinols in } \\
\text { patients with pan- } \\
\text { creatic cancer in } \\
\text { the palliative state }\end{array}$ & $\begin{array}{l}\text { University of Copen- } \\
\text { hagen }\end{array}$ & THC dronabinol & Oral & n.a. (ongoing) \\
\hline EudraCT2019-001382-32 & $\begin{array}{l}\text { Pain associated with } \\
\text { cancer }\end{array}$ & $\begin{array}{l}\text { Beckley Canopy } \\
\text { Therapeutics Ltd. }\end{array}$ & $\mathrm{CBD}$ & Oral & n.a. (2019 completed) \\
\hline EUdraCT2011-005289-39 & $\begin{array}{l}\text { THC in behavioral } \\
\text { problems and pain } \\
\text { in dementia }\end{array}$ & $\begin{array}{l}\text { Radboud University } \\
\text { Medical Center }\end{array}$ & THC Namisol & Oral & II (ongoing) \\
\hline NCT04099355 & $\begin{array}{l}\text { Dronabinol on post- } \\
\text { surgical pain }\end{array}$ & $\begin{array}{l}\text { New York State Psy- } \\
\text { chiatric Institute }\end{array}$ & THC dronabinol & Oral & I (2023) \\
\hline NCT03661892 & $\begin{array}{l}\text { Decreasing use of } \\
\text { opioids in breast } \\
\text { cancer subjects } \\
\text { with bone mets }\end{array}$ & $\begin{array}{l}\text { University of Ari- } \\
\text { zona }\end{array}$ & Syndros dronabinol & Oral (With high-fat) & $1(2020)$ \\
\hline NCT03522324 & $\begin{array}{l}\text { Pain research: inno- } \\
\text { vative strategies } \\
\text { with marijuana }\end{array}$ & $\begin{array}{l}\text { University of Colo- } \\
\text { rado, Boulder }\end{array}$ & Cannabis edible & Oral & Obs (2022) \\
\hline NCT03215940 & $\begin{array}{l}\text { Treatment of chronic } \\
\text { pain }\end{array}$ & University of Utah & THC dronabinol & Oral & I (2022) \\
\hline NCT03675971 & $\begin{array}{l}\text { Cannabinoids to } \\
\text { obviate pain } \\
\text { experiment after } \\
\text { knee replacement }\end{array}$ & Unity Health Toronto & $\mathrm{CBD}$ & Oral & II/III (2022) \\
\hline NCT04387617 & $\begin{array}{l}\text { Postoperative pain } \\
\text { after ureteroscopy } \\
\text { for urinary calculi }\end{array}$ & Mayo Clinic & CBD & Oral & II (2021) \\
\hline $\begin{array}{l}\text { NCT04729179 } \\
\text { EudraCT2019-002394-59 }\end{array}$ & $\begin{array}{l}\text { Medical cannabis for } \\
\text { fibromyalgia }\end{array}$ & $\begin{array}{l}\text { Marius Henriksen, } \\
\text { Frederiksberg Uni- } \\
\text { versity Hospital }\end{array}$ & CBD & Oral & III (2023) \\
\hline NCT04587791 & $\begin{array}{l}\text { CBD in opioid use } \\
\text { and chronic pain }\end{array}$ & Yale University & CBD & Oral & Safety (2020) NYR \\
\hline NCT03984565 & $\begin{array}{l}\text { Sublingual canna- } \\
\text { bidiol for chronic } \\
\text { pain }\end{array}$ & $\begin{array}{l}\text { Staci Gruber, Ph.D., } \\
\text { Mclean Hospital }\end{array}$ & CBD & Sublingual & II (2020) NYR \\
\hline NCT01595620 & $\begin{array}{l}\text { Human experimental } \\
\text { pain models }\end{array}$ & Yale University & THC & IV & n.a. $(2021)$ \\
\hline EudraCT 2019-001861-33 & $\begin{array}{l}\text { Cannabis-opioid } \\
\text { interaction in the } \\
\text { treatment of fibro- } \\
\text { myalgia pain }\end{array}$ & $\begin{array}{l}\text { Leiden University } \\
\text { Medical Center }\end{array}$ & Cannabis & Inhalation & Ongoing \\
\hline
\end{tabular}

$I V$ intravenously, n.a. not available data, NYR not yet recruiting, obs observational study

${ }^{a}$ Some studies are now suspended or relocated due the Covid-19 pandemic. The date of search update for the reported studies was May 8th, 2021

after surgery, as well as in acute and chronic diseases, are reported in Table 3

GW Pharmaceutics have recently filed a series of patents on the oral delivery of cannabinoids, both as single agents and in combinations [102-104]. The cannabinoids are preferably loaded into micelles using non-ionic surfactants (e.g., macrogolglycerol hydroxystearate) and administered as oral aqueous formulations. Improved biodistribution in healthy subjects of 2-fold $C_{\max }$ and 1.21-fold AUC have been reported. Moreover, an oral gel formulation has been reported in which cannabinoids are melted with poloxamers (poloxamer 124 and poloxamer 188 are preferred) and then filled into HPMC, gelatin and soft-gelatin capsules.

An oral CBD oil formulation (CardiolRx ${ }^{\mathrm{TM}}$ ) is being developed by Cardiol Therapeutics Inc. (CA). The FDA very recently (September 2020) approved an IND application for a Phase II/III trial for this oral formulation, taken with food, to prevent cardiovascular complications in hospitalised patients with a confirmed diagnosis of SARS-CoV-2 infection in the previous $24 \mathrm{~h}$ and who have pre-existing 
cardiovascular disorders and/or significant risk factors for cardiovascular disorders (NCT04615949). Important to note that, very recently, the 7-hydroxy-CBD was found to be able to promotes the host innate immune response and thus may be proposed as potential therapeutic treatment for COVID19 at early stages of infection [105].

An interesting, although challenging, technology that is based on co-crystallisation has been developed by Artelo Biosciences Inc (USA) in order to improve the bioavailability of CBD [106]. The co-crystals consist of the active pharmaceutical ingredients and one, or more, unique crystalline co-formers (which can be another active substance) that modify the material properties whilst retaining the intrinsic pharmacological activities of the drugs [107].

Co-crystallisation is a useful, although rarely exploited, method for improving the physicochemical properties of constituent active pharmaceutical ingredients and for overcoming problems with drugs by improving their biopharmaceutical properties. In an interesting article, Almansa et al. have described the potential role of this approach in the search for a multimodal therapy for pain treatment [108].

Artelo Biosciences have made use of the co-former tetramethylpyrazine (also called ligustrazine), a plant-derived compound from the Ligusticum striatum DC (=L. chuanxiong) that is widely used in Chinese medicines. Preclinical development is focused on post-traumatic stress disorder, inflammatory bowel disease, stroke, and rare diseases.

Regarding the solid-state behaviour of CBD, amorphous $\mathrm{CBD}$ is more soluble in water than its crystalline form, but it tends to recrystallise. In order to stabilise amorphous CBD, three strategies based on different compounds were proposed; cyclodextrins, mesoporous silica and water-soluble polymers were processed using different preparation techniques. Then, several parameters (drug loading, aqueous solubility, final stability, ease of translating for large-scale drug production) were evaluated for each formulation, showing that all the approaches stabilised the CBD amorphous form for at least two months. However, other characteristics, such as drug loading, dissolution rate and suitability for scale up, differ for each approach [109].

A tablet formulation to improve the bioavailability of CBD, designated as Arvisol ${ }^{\circledR}$, is being developed by Echo Pharmaceuticals (NL). This formulation is designed for the treatment of neurological diseases, including Rett syndrome, schizophrenia, and epilepsy $[110,111]$. Tablets and granulates are prepared, in a model composition, using the wet granulation process by combining CBD $(6.7 \% \mathrm{w} / \mathrm{w})$, sucrose monolaurate $(13.3 \%)$, ascorbic acid $(0.7 \%)$ and lactose. The granulate was blended with excipients and compressed into tablets for oral use. Pharmacokinetic evaluations showed a relevant (cited to be 100-fold) improvement in AUC, compared to capsules loaded with CBD powder, for a $200 \mathrm{mg}$ dosage of the drug.
Another interesting, but expensive and highly challenging, approach is the chemical modification of CBD in order to improve solubility, using the synthesis of prodrugs or conjugates that link two molecular entities. Kalytera Therapeutics, now Claritas Pharmaceuticals (IL), is developing a $\mathrm{CBD} /$ naproxen conjugate compound for the treatment of acute and chronic pain. The non-psychotropic, oral analgesic has a cannabinoid component that acts as a cannabinoid receptor agonist, and targets the alpha3 glycine pain receptor in the spinal cord. When activated, the alpha3 glycine receptor blocks pain signals from being sent to the brain. The naproxen component blocks the synthesis of the pain-inducing prostaglandin molecule PGE2 (dinoprostone). Although the initial route of administration will be oral, Claritas is also seeking to develop an intravenous formulation of the compound. Early research is underway in the USA [112, 113].

The company was also developing other synthetic derivatives of cannabinoids, (in particular CBD) that were structurally modified to enhance solubility in water for improved potency (e.g. as phosphonate prodrugs) [114]. These were being developed for the treatment of bone fracture, but the company has recently discontinued the development of three candidates.

An oral formulation of CBD has also been evaluated for use as prophylaxis for Graft-Versus-Host Disease after allogeneic haematopoietic stem-cell transplantation. Clinical development is underway in Australia and Israel (NCT03840512, ACTRN12619000623190).

Diverse Biotech Inc. (USA) is also developing CBD conjugates for the treatment of cancers. The cannabinoid is the core component, and new chemical entities that are theoretically more efficacious and less toxic are in development via combinations with other targeted molecules through conjugate chemistry [115]. Preclinical development is underway in the USA.

So-called TurboCBD ${ }^{\mathrm{TM}}$ has been proposed by Lexaria Bioscience Corp. (CA). Capsules with the following composition, CBD in hemp oil added to a high proportion of American ginseng and Ginko biloba extracts, have been made. Formulation is based on DehydraTECH ${ }^{\mathrm{TM}}$ delivery technology, by Poviva Corp., which includes a patented process by which long-chain fatty acids, high in oleic acid, are associated with CBD using a dehydration process procedure [116].

The results of a clinical trial on TurboCBD ${ }^{\mathrm{TM}}$, in comparison with the CBD in hemp oil, on 12 healthy participants in a double-blind, placebo-controlled, cross-over design have been reported by Patrician et al. [117]. The incorporation of TurboCBD ${ }^{\mathrm{TM}}$ improved CBD bioavailability by $111 \%$, compared with an equivalent generic $90 \mathrm{mg}$ dose, but not with a half dose. The authors stated that TurboCBD ${ }^{\mathrm{TM}}$ also exhibited a potential advantage over CBD in gelatin matrix pellets, with a $C_{\max }$ time of $120 \mathrm{~min}$, in comparison to $3-3.5 \mathrm{~h}$. 
The mechanism for this effect is unclear, but the authors believe that the presence of long-chain fatty acids, associated with $\mathrm{CBD}$ using the dehydration processing technique, allowed higher concentrations of CBD to enter the circulatory system, perhaps by bypassing first-pass liver metabolism. Indeed, lower metabolite levels of 7-hydroxy-CBD and 7-CBD-carboxylic acid were detected (although in very few subjects). A clinical trial in which TurboCBD ${ }^{\mathrm{TM}}$ is used as dietary supplementation is ongoing (NCT03295903).

The DehydraTECH ${ }^{\mathrm{TM}}$ delivery technology has recently also been proposed as a means for the transdermal delivery of CBD and other lipophilic substances [118].

An oral formulation of CBD as a nanoemulsion has also been reported in a recent patent by the same authors who describe a composition made up of lactose monohydrate powder, as a base substrate, and highly purified CBD crystals and sunflower oil in a 1:1 ratio [119]. A surfactant, polysorbate 80 , has also been added to an aqueous phase of quillaja, obtained from Quillaja saponaria. This extract is rich in saponins, mainly surfactants, but has a wide range of biological activities and is approved for use as a food additive in many countries and as a vaccine adjuvant [120]. Furthermore, a homogenisation step is used to achieve the size reduction required.

A CBD oral formulation (named PTL 101) for the treatment of intractable epilepsy is being developed by MMJ PhytoTech Ltd (AU). PTL 101 capsules are made from a proprietary formulation developed using Satipharm's Gelpell ${ }^{\circledR}$ product technology [121]. In detail, doses of 10 to $100 \mathrm{mg}$ $\mathrm{CBD}$, dissolved in oil, were added to a hot gelatin solution, then cooled and dried to produce gelatin pellets, which were finally loaded into hard gastro-resistant capsules. The soluble gelatin polymer was used to promote dispersion by producing a microemulsion in situ, which is expected to enhance the bioavailability of the $\mathrm{CBD}$, while avoiding punctual irritation of the gastric mucosa. Clinical development, underway in Israel (NCT02987114), has demonstrated interesting reductions in seizures in paediatric patients with treatment-resistant epilepsy over a 12-week period [122]. A pharmacokinetic study showed that the CBD, administered under fed conditions, provides a relevant improvement in AUC (134\% for $100 \mathrm{mg}$ dose), compared to an oromucosal spray [123].

CBD oral formulations have been developed by Stero Biotechs as an adjunctive treatment to reduce side effects and the need for steroid therapy [124]. The product (ST 101) is made up of CBD dissolved in olive oil at a concentration of $2.5 \%$ and has been evaluated for autoimmune hepatitis and Crohn's disease treatments (NCT04056442). Another CBD product is being developed for the treatment of chronic urticaria. Clinical development is underway in Israel.

A particular host matrix for the incorporation of CBD, in the form of an inclusion complex with $\beta-C D$, has been fabricated by combining pectin and honey to obtain nontoxic 3D printable inks that form solid structures upon drying. The fate of CBD was evaluated in different environments (simulated gastric fluid, simulated intestinal fluid, and simulated colonic fluid). The highest CBD release rates were obtained in the simulated colonic environment medium, in which pectin is expected to be fully degraded by enzymes. The interest in this $3 \mathrm{D}$ printing process relies on the possibility of fabricating personalised food grade CBD dosage forms [125].

Studies of the inclusion complexes of native CDs with CBD showed that the water solubility increased thanks to the formation of 1:1 $(\alpha-\mathrm{CD}), 2: 1(\beta-\mathrm{CD})$ and 2:1 ( $\gamma$-CD) hostguest inclusion complexes [126]. The energetic and thermodynamic aspects of the CBD-CD interaction in aqueous solution were deeply analysed and the authors observed that the degree of CBD-CD complexation at four temperatures values depended on the medium; it is weaker at increased temperatures, except for CBD- $\gamma$-CD whose complexation is practically insensitive to temperature changes, indicating that the $\gamma$-CD complex is more stable than the others [127].

An interesting recent analysis of five oral CBD formulations has been described by Williams [128]. A dose of 30 $\mathrm{mg}$ CBD was tested as a solid in suspension in water (code 203), with oil and starch (code 472), in an oil solution (code 178), as a liquid formulation with Quillaja extract (20\% CBD, code 340) and with gum Arabic (5\% CBD code 707). This last formulation appeared to be superior to most of the others. Indeed, it generally evoked the fastest $T_{\max }$, greatest $C_{\max }$, and largest AUC. This is also confirmed in light of the majority of the data available in literature on oral CBD. Only when CBD was formulated in a self-emulsifying delivery system (see next section) was higher $C_{\max }$ obeserved despite a smaller dose (25 mg) [129]. Interestingly, Williams also presented the majority of available pharmacokinetic data now available (year 2020) for orally administered cannabinoids. This information is considered useful for the reader and is fully extracted and shown in Table 4 .

A pain therapeutic drug that contains $\mathrm{CBD}$ and THC is being developed by AusCann Group Holdings Ltd (AU), with a clinical trial underway in Australia. In April 2020, a Phase I trial was initiated to evaluate the pharmacokinetics of a single dose of two formulations of an orally administered CBD/THC combination (ACTRN12620000462987). The randomised, open-label crossover trial has enrolled 28 fed healthy volunteers in Australia, where capsules containing (ratio 1:1) $2.5 \mathrm{mg}$ and $10 \mathrm{mg}$ formulations were administered. Two patents on solid cannabinoid compositions have recently been filed by AusCann [130, 131] where solid self-emulsifying compositions, which contain either a single or a blend of cannabinoids, were made up of medium chain triglycerides (MCT), surfactant Cremophor EL and colloidal anhydrous silica. A chewing gum 
Table 4 Comparison of the pharmacokinetic parameters of preparations proposed by Williams et al with previously reported studies of ingestible cannabinoids

\begin{tabular}{|c|c|c|c|c|c|c|c|c|}
\hline Formulation, administration, CBD single dose (mg) & $T_{\max }(\mathrm{h})$ & $C_{\max }(\mathrm{ng} / \mathrm{mL})$ & $\begin{array}{l}\mathrm{AUC}_{\mathrm{o}-\mathrm{t}} \\
(\mathrm{h} \cdot \mathrm{ng} / \\
\mathrm{mL})\end{array}$ & $\begin{array}{l}\mathrm{AUC}_{0-\text { inf }} \\
(\mathrm{h} \cdot \mathrm{ng} / \mathrm{mL})\end{array}$ & $T_{1 / 2}(\mathrm{~h})$ & $K_{\mathrm{a}}(1 / \mathrm{h})$ & $K_{\mathrm{e}}(1 / \mathrm{h})$ & $\overline{V_{\mathrm{d}}(\mathrm{L})}$ \\
\hline Oral capsule (CBD + THC) $5.4 \mathrm{mg}$ & 0.99 & 0.93 & 4.35 & & & & & \\
\hline Oral capsule (CBD + THC) $5.4 \mathrm{mg}$ & 1.0 & 0.95 & & & & & & \\
\hline GW oral capsule $(\mathrm{CBD}+\mathrm{THC}) 10 \mathrm{mg}$ & 1.27 & 2.47 & 5.76 & 6.03 & 1.09 & & & \\
\hline Oral capsule $(\mathrm{CBD}+\mathrm{THC}) 10 \mathrm{mg}$ & 1 & 2.1 & 6.9 & & & & & \\
\hline PTL101 CBD oral capsule $10 \mathrm{mg}$ & 3 & 3.22 & 9.64 & 10.31 & 2.95 & & 0.1 & \\
\hline PTL401 (CBD + THC) oral capsule $10 \mathrm{mg}$ & 1.25 & 2.94 & 9.85 & 10.52 & 3.21 & & 0.29 & \\
\hline Oral capsule MCT-CBD $25 \mathrm{mg}$ & 3.0 & 3.05 & 9.51 & 19.23 & & & & \\
\hline Oral capsule SEEDS-CBD $25 \mathrm{mg}$ & & 13.53 & 27.15 & 32.63 & & & & \\
\hline Caliper Foods CBD water soluble $30 \mathrm{mg}$ & 0.9 & 2.82 & 6.80 & 7.94 & 2.54 & 1.68 & 0.66 & \\
\hline Caliper Foods CBD lipid soluble $30 \mathrm{mg}$ & 1.5 & 0.65 & 1.51 & 1.64 & 2.30 & 1.14 & 0.72 & \\
\hline Preparation 178 CBD $30 \mathrm{mg}$ & 3.29 & 2.20 & 4.58 & - & - & 0.32 & - & - \\
\hline Preparation 203 CBD $30 \mathrm{mg}$ & 3.39 & 1.29 & 2.30 & - & - & 0.24 & - & - \\
\hline Preparation 340 CBD $30 \mathrm{mg}$ & 1.28 & 3.54 & 7.81 & 13.81 & 2.20 & 1.19 & 0.40 & 7428 \\
\hline Preparation 472 CBD $30 \mathrm{mg}$ & 1.53 & 2.88 & 6.32 & 9.96 & 5.18 & 1.87 & 0.27 & 20,178 \\
\hline Preparation 707 CBD $30 \mathrm{mg}$ & 0.70 & 5.57 & 9.12 & 10.77 & 1.42 & 1.43 & 0.56 & 8024 \\
\hline PTL101 CBD oral capsule $100 \mathrm{mg}$ & 3.5 & 47.44 & 150 & 153 & 3.59 & & & \\
\hline$\left(\right.$ Epidiolex $\left.^{\circledR}\right) 200 \mathrm{mg}$ & 2.3 & 148.0 & 449 & 474 & 8.58 & & & 4105 \\
\hline$\left(\right.$ Epidiolex $\left.^{\circledR}\right) 200 \mathrm{mg}$ & 2.5 & 200.0 & 671 & 600 & 15.5 & & & 6661 \\
\hline$\left(\right.$ Epidiolex $\left.^{\circledR}\right) 200 \mathrm{mg}$ & 2.0 & 172.0 & 530 & 522 & 14.6 & & & 7778 \\
\hline$\left(\right.$ Epidiolex $\left.^{\circledR}\right) 200 \mathrm{mg}$ & 2.5 & 155.0 & 532 & 601 & 13.1 & & & 6016 \\
\hline$\left(\right.$ Epidiolex $\left.^{\circledR}\right) 200 \mathrm{mg}$ & 2.5 & 153.0 & 464 & 499 & 11.2 & & & 5800 \\
\hline GW oral CBD capsule $400 \mathrm{mg}$ & 3 & 181.2 & 704 & & & & & \\
\hline GW oral CBD capsule $400 \mathrm{mg}$ & 1.5 & 114.2 & 482 & & & & & \\
\hline$\left(\right.$ Epidiolex $\left.^{\circledR}\right) 750 \mathrm{mg}$ & 4.0 & 187.0 & 1077 & 1190 & 39.7 & & & \\
\hline GW oral CBD capsule $800 \mathrm{mg}$ & 3 & 221.1 & 867 & & & & & \\
\hline GW oral CBD capsule $800 \mathrm{mg}$ & 4 & 157.1 & 722 & & & & & \\
\hline GW oral solution $1500 \mathrm{mg}$ & 4 & 292.4 & 1517 & 1618 & 14.43 & & & 20,963 \\
\hline GW oral solution $1500 \mathrm{mg}$ & 3.5 & 335.4 & 1987 & 2198 & 30.33 & & & 34,101 \\
\hline$\left(\right.$ Epidiolex $\left.^{\circledR}\right) 1500 \mathrm{mg}$ & 6.13 & 524.5 & 2650 & 2713 & & & & \\
\hline $\mathrm{GW}$ oral solution $3000 \mathrm{mg}$ & 5 & 533.0 & 2669 & 2802 & 14.39 & & & 23,357 \\
\hline GW oral solution $4500 \mathrm{mg}$ & 5 & 722.1 & 3215 & 3426 & 16.61 & & & 36,575 \\
\hline$\left(\right.$ Epidiolex $\left.^{\circledR}\right) 4500 \mathrm{mg}$ & 4.07 & 426.9 & 2339 & & & & & \\
\hline GW oral solution $6000 \mathrm{mg}$ & 5 & 782 & 3696 & 3900 & 15.42 & & & 42,849 \\
\hline
\end{tabular}

The Table appeared in the article by Williams et al: "Comparison of Five Oral Cannabidiol Preparations in Adult Humans: Pharmacokinetics, Body Composition, and Heart Rate Variability" Pharmaceuticals 2021, 14, 35. Permitted reproduction under the terms and conditions of the Creative Commons Attribution (CC BY) license (https://creativecommons.org/licenses/by/4.0/)

composition with controlled release that combines the two cannabinoids (ratio 1:1) at a dosage of $5 \mathrm{mg}$ is being developed by Axim Biotechnologies Inc. (USA) for the treatment of patients suffering from multiple-sclerosis-associated pain and spasticity, Parkinson's disease, dementia, restless leg syndrome and post-herpetic neuralgia. Clinical development is underway in the USA, as is preclinical development for post-herpetic neuralgia and psychotic disorders. Nevertheless, no recent reports of development for Phase I developments have been identified. Patents on the co-administration of cannabinoids and opioid agonists and/or antagonists have also been reported [132-134].

An analogous orally administrable formulation has also been filed by Greenspoon [135], as AX 1505. This is a sustained-release, floating-capsule phytocannabinoid-based therapeutic, developed by Axim Biotechnologies for the treatment of Crohn's disease. Preclinical development is underway in the USA, but, as of December 2020, no new reports of development have been identified. 
An oral, fixed-dose combination of CBD and THC, designated as BCT 521, has been investigated by Beckley Canopy Therapeutics (UK); a Phase I/II trial has been completed and an evaluation of the efficacy and safety for pain associated with cancer in patients who have already received standard-of-care treatment with opioids has been performed (EudraCT2019-001382-32; BCT-521-201). The randomised, double-blind, placebo-controlled trial was initiated in November 2019 and enrolled 260 patients in Poland. The primary endpoint is a change in the average pain score over $24 \mathrm{~h}$ from the start of titration to the end of treatment.

A CBD:THC fixed-dose combination is also being developed by Knop laboratories (Chile) for the treatment of fibromyalgia. The candidate is formulated as an oral liquid, containing $1 \mathrm{mg}$ of THC and $0.45 \mathrm{mg}$ of CBD per drop. Clinical development is underway in Chile (Phase II) (NCT04239469).

A particular CBD:THC formulation is being developed by Intec Pharma (IL) for the treatment of various indications including pain and fibromyalgia. The therapeutic product will be developed utilising a multi-layered, biodegradable, gastro-retentive drug formulation named Accordion Pill ${ }^{\mathrm{TM}}$. Accordion Pill ${ }^{\mathrm{TM}}$ technology is a polymeric platform that encases a drug preparation and is folded into an accordionlike shape in a standard-sized regular oral capsule [136]. The cannabinoids are emulsified as nano or micro self-emulsifying delivery system (see next section) with a caprylocaproyl macrogolglyceride surfactant, which is also an efficacious intestinal permeation enhancer [137], in a mixture of hydroxypropyl cellulose and oil, and are then dried to a dry film (inner layer). A swelling membrane composed of PEG, hydroxypropyl cellulose and polymethacrylate polymers, a frame film (polymethacrylate polymers and PEG) with a supra-outer film containing THC-CBD and celluloses constituted the full structure of the accordion. Furthermore, an assembly process in which the different layers are stacked and assembled together in a precise order is required. The final laminates are folded in an accordion-like configuration using a folding apparatus and are inserted into a hard-gelatin capsule after folding. After the capsule dissolves in the stomach, the accordion unfolds and can remain in the stomach for extended periods of time, beyond those observed with standard capsules or tablets. While it is in the stomach, the drug is released in a continuous and controlled manner, which optimises absorption into the blood stream. As a result, it is thought that the Accordion Pill ${ }^{\mathrm{TM}}$ formulation will address the disadvantages that are associated with cannabinoid therapies, such as delayed onset and short duration of effect, drug exposure variability, and that it may significantly increase patient compliance due to less repeated dosing. Clinical development is underway in Israel [138].

Hardy and colleagues have recently described (clinical trial ACTRN12619000037101) a placebo-controlled ongoing clinical trial to evaluate the efficacy, safety and acceptability of $1: 1 \mathrm{THC} / \mathrm{CBD}$ for symptom relief in advanced cancer patients $(n=150)$ [139]. An oral oil formulation was administered at a concentration of $10 \mathrm{mg} / \mathrm{mL}$ and a dosage range from $2.5 \mathrm{mg}$ to $30 \mathrm{mg} /$ day for a maximum of 28 days. The primary outcome is the change from baseline as measured by the Edmonton Symptom Assessment Scale total symptom distress score at Day 14. It assesses both physical and psychological symptoms, plus general wellbeing. Recruitment is expected to continue until the end of 2021.

\subsubsection{Nanotechnology-Based Approaches for Oral Delivery}

In general, due to the high lipophilicity of cannabinoids, encapsulation in tailored nanosystems may potentially achieve several goals, such as a gradual release, flavour masking, superior oral bioavailability and higher physical stability than conventional formulations [140]. Although several strategies based on known technologies such as liposomes, polymeric nanoparticles etc. are still debating and in preclinical tests, one of the most interesting and profitable approach is represented by the self-emulsifying systems.

Self-emulsifying drug delivery systems (SEDDS) have been in development since the 1980s and have improved to overcome the low bioavailability and poor solubility of hydrophobic drugs, such as CBD. SEDDS are lipid-based nanosystems that can be easily formed by simply mixing drug, lipids and a relatively high concentration of emulsifiers, usually with one or more hydrophilic co-solvents/coemulsifiers. This mixture produces oil-in-water emulsions or microemulsions upon gentle agitation, followed by dilution with an aqueous phase. SEDDS are therefore interesting from an industrial point of view, since large batches can be prepared without any sophisticated technology. This approach is employed with success and is a commercially available system for BCS Class II (low solubility, high permeability) and class IV (low solubility, low permeability) drugs [141].

For a wider recent view of these systems, the reader can find an entire issue of Advanced Drug Delivery Reviews devoted to this topic [142]. This technique is one of the most common approaches in cannabinoid delivery and its applications will be described below.

SEDDS typically produce emulsions with a droplet size of $300 \mathrm{~nm}$, while the droplet size is below $200 \mathrm{~nm}$ in selfnanoemulsifying drug delivery systems (SNEDDS) and a lower quantity of surfactants is used [143]. SNEDDS have been used as advanced lipid-based formulations to improve solubility and contribute to the lymphatic absorption of cannabinoids; some of these approaches have been patented in recent years, differing from each other in composition and 
manufacturing procedures [144-146]. Indeed, the composition of SEDDS greatly influences the pharmacokinetic behaviour of the encapsulated compound. Thus, several studies have focused on tuning and comparing cannabinoidSEDDS and SNEDDS formulations. For example, medium and long chain triglycerides have recently been incorporated into SNEDDS in order to evaluate their effect on the oral absorption of major cannabinoids. Nevertheless, an investigation in an in vivo preclinical model in healthy rats showed that these differences are masked after the incorporation of MCT and LCT into SNEDDS [147].

The lipids used in SNEDDS formulations may also have an impact on the pharmacokinetics of associated drugs, as in the case of $\mathrm{CBD}$, which was orally administered to healthy rats in SNEDDS and compared to a formulation in sesame oil. The nano-formulation had a rapid onset of absorption but oily preparation was characterised with a prolonged absorption phase lasting 4-6 h [148]. The differences in the physicochemical properties (size, surfactants) of vehicles surely affected the cannabinoid solubilisation in gastrointestinal tract.

SNEDDS have also been compared to a CBD-containing floating gastro-retentive tablet that is based on an egg albumin matrix, gas-generating agents, and surfactants. Once administered to rats, the gastro-retentive dosage form showed a prolonged absorption phase with a substantial increase in bioavailability, compared to the CBD-lipid formulation, thanks to gradual drug release. Indeed, dissolution into the aqueous environment of the stomach is a rate limiting step for $\mathrm{CBD}$, but when it occurs under sink conditions, the absorption is less dependent on this process [149].

To enhance the bioavailability of cannabinoids, the alkaloid piperine has been combined with SNEDDS and orally administered to rats. The results showed an approximate 2.5fold increase in the oral bioavailability of CBD with piperine dissolved in SNEDDS (once-daily dose) compared to CBD-SNEDDS alone. Nevertheless, there was no significant difference in CBD absorption with acute or chronic administration of piperine [150].

Other SEDDS formulations, in which antioxidants were added to the mixture, have been encapsulated in a gelatin or hydroxyl-propyl-methylcellulose capsule shell and orally administered to dogs. Cannabinoids in SEDDS achieved significantly higher oral bioavailability than the same dose administered orally in the form of an oil solution or a commercially available THC tablet. Nevertheless, when administered to evaluate the effects on the management of neuropathic pain, the cannabinoids in SEDDS achieved significantly lower pain level scores than the same dose administered orally in the form of an oil solution [151].

Despite interesting results in animal models, reported cannabinoid pharmacokinetics have proven to be modeldependent, and not uniform among research groups, and affected by the vehicle of administration. Thus, in order to investigate the oral absorption processes of CBD when given in oral formulations in healthy volunteers, CBD was administered in different formulations (powder form, dissolved in sesame oil and in SNEDDS) in a three-way, blind, cross-over single administration study on 12 subjects. The administration of CBD in both lipid-based vehicles resulted in a significant increase in $C_{\max }$ and AUC, compared to the powder form. Moreover, CBD in SNEDDS was rapidly absorbed in all volunteers, as opposed to the sesame oil group, which showed greater variability and less predictable pharmacokinetic behaviour [152]. These results again highlight the need for a standardised CBD oral formulation to reduce its high intra- and inter-subject absorption variability in humans.

A novel CBD-containing SEDDS formulation has been developed using food emulsifiers, edible vegetable oils and fatty acids, according to the VESIsorb ${ }^{\circledR}$ technology, developed by Vesifact AG (Switzerland), which has previously increased the oral bioavailability of lipophilic molecules [153]. CBD has been used as a hemp-extract and SEEDS formulation, with the mean diameter of the droplets formed being between 40 to $50 \mathrm{~nm}$ and was compared to the same extract diluted with MCT oil. The authors evaluated the pharmacokinetic profile of SEDDS in a randomised, doubleblind, cross-over design in 16 healthy volunteers under fasting conditions. A single oral administration of CBD-SEDDS led to an increase in both $C_{\max }$ and $\mathrm{AUC}_{0-8 \mathrm{~h}} / \mathrm{AUC}_{0-24 \mathrm{~h}}$ compared to the reference formulation, while $T_{\max }$ was shorter for SEDDS than for the MCT-form [129].

Nanotechnology formulations for cannabis-based extracts have been also proposed for oral and sublingual administrations. Nanoemulsions, starting from oil droplets of predetermined sizes, have been prepared in the form of readily water-dispersible fine powders by lyophilisation. The dry fine lyophile powder is able to recover the oil droplets in approximately the same size, upon contact with water [154]. To reach this goal, the formulations should contain at least one cryoprotectant (such as sucrose, trehalose, or mannitol), at least one carbohydrate (such as maltodextrin and carboxymethyl cellulose) in great percentage and, in a proportion less than 5\% at least one surfactant (such as ammonium glycyrrhizinate). The controlled combination of these components allows tailored nanodroplets to be produced. From a technological point of view, an interesting comparison between microfluidic and ultrasound application to produce cannabinoid nanoemulsion has been recently proposed by Fathordoobady et al. [155, 156].

\subsubsection{Minor Cannabinoids and Derivatives}

Cannabichromene is a non-psychoactive, naturally occurring phytocannabinoid compound that is being selected by Cannabics Pharmaceuticals (USA) for the treatment of cancers, 
including gastrointestinal cancer. Preclinical development is underway in Israel.

Cannabics Pharmaceuticals is also the proprietor of patents that describe a method of profiling the patient microbiome and cannabinoids [157], in order to select personalised cannabinoid-based therapy for subjects diagnosed with cancer [158].

Regarding formulations proposed by Cannabics Pharmaceuticals, a supercritical $\mathrm{CO}_{2}$ cannabinoid extract (that is then decarboxylated) (with $1.2-8 \%$ active substances) has been formulated with various amounts of mono and diglycerides, coconut oil, lecithin and Iota carrageenan and administered in capsules. This approach has been proposed for the treatment of cachexia and anorexia in advanced cancer patients and their quality of life was monitored $(n=25)$ (NCT02359123). The trial concluded in 2018 [159].

Zyus Life sciences Inc. (CA) has been evaluating oral formulations comprising a mixture of THC, CBD and CBC, that are dispensed as pills, tablets or soft-gel capsules administered with high-fat food in amounts aimed at managing or treating pain in individuals. This study confirmed that CBC significantly prolongs the effect of CBD + THC in a preclinical rat model of neuropathic pain [160].

Synthetic derivatives of CBD have been developed by VivaCell Biotechnology (ES) and proposed for oral formulations. One drug candidate, named EHP 101 (previously known as VCE-004.8, see Fig. 2) is a stable derivative of the CBD quinone (HU-331) and is administered as a liquid formulation. This candidate was proposed for the treatment of scleroderma and perhaps other fibrotic diseases [161, 162]. Safety and tolerability clinical trials (NCT03745001, NCT04166552) are currently ongoing.

Another promising minor phytocannabinoid derivative, EHP 102 (previously known as VCE-003.2, see Fig. 2), which is derived from $\mathrm{CBG}$, and its oral formulation are being developed by Emerald Health Pharmaceuticals (USA) (now called Skye Bioscience) for the treatment of Huntington's disease and Parkinson's disease. CBG is proposed as a cannabinoid with anti-inflammatory action, and there is some evidence of neuroprotection. This compound has been modified to be more effective than CBG as it can affect the peroxisome proliferator-activated receptor gamma (PPAR $\gamma$ ), which is a key molecular target for the treatment of Huntington's disease, as well as being able to target other physiologic pathways involved in neuronal survival [163, 164]. Preclinical development is underway in the USA where a lipid formulation of VCE-003.2 in corn oil is being used for oral administration $[165,166]$.

In addition to Sativex and Epidiolex, GW Pharmaceuticals is developing oral formulations of two minor cannabinoids, cannabidivarin (GWP42006) and $\Delta$ 9-tetrahydrocannabivarin (GWP 42004). The former has been proposed for the treatment of epilepsy, autism spectrum disorders, neuropathic pain and Prader-Willi syndrome. Clinical trials involve people with focal seizures (NCT02369471) and, more recently, children and young adults with autism spectrum disorder (NCT03849456). Tetrahydrocannabivarin-9 is an antagonist at the CB1 and CB2 receptors and may also act as a glucagon-like peptide-1 (GLP-1) secretagogue. An oral formulation of $\Delta 9-\mathrm{THCV}$ is in development for the treatment of type 2 diabetes mellitus. Clinical development is underway in the UK and Romania (NCT02053272, EudraCT2013-001140-61) [167].

\subsection{Oral Formulations for Colonic Delivery}

Due to their important antioxidant and anti-inflammatory activity, cannabis and cannabinoids have also been proposed for the treatment of inflammation processes of the intestine, although there is some debate over this use [168, 169]. Cannabis use has been reported to be useful for the symptomatic control of inflammatory bowel disease, and has been reported as successful in the management of abdominal pain, joint pain, cramping, etc. Controlled-release forms are desirable in the attempt to limit the side effects, while significantly reducing the morbidity seen in patients with severe disease.

Furthermore, colon-specific drug-delivery systems can also be proposed for the treatment of a range of local diseases, such as ulcerative colitis, Crohn's disease, chronic pancreatitis, and colonic cancer. Very recently, some patents have appeared (by GW Pharmaceutics and ICD Pharma Ltd USA) proposing the use of cannabinoids in controlledrelease formulations to the intestine using a $\mathrm{pH}$-dependent release polymer [170]. CBD, THC, and other cannabinoids can be loaded into microspheres, made of hydroxypropyl methyl cellulose acetate succinate, in the presence of a poloxamer emulsifier. Further, microspheres can be formulated in suspension for enteric or colonic release, by means of modified cellulose or methacrylate copolymers (Eudragit S100).

Intestinal (small, large and colon) delivery formulations of cannabinoids have also been proposed by Friedman (ICD Pharma Ltd) [171]. Cannabinoids (CBD, THC, and cannabis extracts) were prepared as a hot melt dissolved in a mixture of emulsifiers and solid-matrix-forming agents, such as polymers and waxes. When in contact with body fluids, the solid solutions eroded or swelled into tiny particles (below 100 micron) improving the bioavailability of cannabinoids.

\subsection{Pulmonary Administration}

The most common route of administration for cannabisderived medicines is by inhalation, or smoking. The intrapulmonary administration of cannabinoids is regarded as an effective mode of delivery as it results in the fast onset 
of action (5-10 min), rapid delivery to the central nervous system and high systemic bioavailability. Furthermore, this route of administration is useful for patients who are not in the condition to take oral medications (e.g., in the case of serious nausea and vomiting). To avoid the production of toxic by-products via combustion, vaporisation, which is a technique by which cannabis is heated to the temperature of cannabinoid vaporisation, but below the point of combustion, has been proposed since the 1990s. Different types of vaporiser devices have been marketed; from larger desktop devices, such as the Volcano ${ }^{\circledR}$ or the Arizer Extreme $Q^{\circledR}$, to more portable models, such as the Arizer Solo ${ }^{\circledR}, \mathrm{Crafty}^{\circledR}$ or Mighty ${ }^{\circledR}$ Medic portable vaporisers (Storz-Bickel GmbH, same producer as the Volcano ${ }^{\circledR}$ ) or the Syqe Inhaler, developed by Syqe Medical (IL).

Regarding the evaluation of the analgesic effects of inhaled pharmaceutical-grade cannabis, van de Donk et al. have presented an experimental randomised study in chronic pain patients with fibromyalgia [172] (EudraCT 2019-001861-33). The varieties used were Bedrocan (high THC/low CBD content), Bedrolite (high CBD/low THC content) and Bediol (high THC/high CBD content). All cannabis varieties were vaporised using the Volcano Medic vaporiser for intrapulmonary administration. The pharmacokinetics and pharmacodynamics of three active cannabis varieties were described. It was observed that, when simultaneously inhaled, THC and CBD interact in complex fashions with synergistic pharmacokinetic, but antagonistic, pharmacodynamic interactions. The analgesic efficacy of active treatment was limited to varieties that contained THC and was observed exclusively in the evoked pressure pain model. None of the active treatments were effective in reducing spontaneous pain scores more than the placebo. With inhaled forms of cannabis, the bioavailability is dependent on the size of the vaping chamber, depth of inhalation, breath holding and the concentration of active components.

A combination of dry compressed cannabis standardised in THC and CBD, plus several terpenes, for use as an inhalation product, is in Phase III development for the treatment of pain and inflammation by Tetra Bio-Pharma (CA), while clinical development is underway in Canada and the USA (NCT04042545) [173]. The CAUMZ ${ }^{\mathrm{TM}}$ Kit comprises two coated capsules that mainly contain $17 \mathrm{mg}$ THC and 5 mg CBD (PPP001) in a PVC/aluminium blister pack, and a handheld Mighty ${ }^{\circledR}$ Medic portable vaporiser. The drug component and device component can be assembled via the labelling of each component. The vapour to be inhaled is generated in a titanium pipe in the vaporiser via the heating of PPP001.

A Phase II trial in which inhaled PPP001 is evaluated against immediate-release oral opioids (morphine sulfate) for the management of breakthrough cancer pain is ongoing in the USA (NCT03564548). Another Phase III trial is investigating patients with advanced cancer suffering from cachexia. Inhaled PPP001, in addition to palliative care management, will be assessed to ascertain whether these patients experience improvements in functional status, as a surrogate endpoint for survival, and quality of life (NCT04001010). An inhaled formulation of dronabinol, designated as HCC011, is also being proposed by Tetra Bio Pharma as an adjunctive treatment for hepatocellular carcinoma. Clinical development is underway in Canada.

Different from the vaporisation of cannabis fumes, an inhaled dry powder formulation of dronabinol is being developed by MannKind Corp. (USA) and Receptor Life Sciences (USA) and is based on the Technosphere ${ }^{\circledR}$ platform [174-176]. This technology is centered on Technosphere particles, which are formed by the self-assembly of crystals of a proprietary excipient 3,6-bis( $N$-fumaryl-4-aminobutyl)2,5-diketopiperazine (FDKP). Cannabis extracts (mainly dronabinol) dissolved in ethanol in the presence of phospholipids and other excipients were spray-dried with FDKP microcrystallites obtaining formulations containing up to $30 \%$ of the cannabinoid. Clinical indications are for the treatment of chemotherapy-induced nausea, vomiting and anorexia in patients with acquired immunodeficiency syndrome. The Technosphere ${ }^{\circledR}$ platform is characterised by the ultra-rapid onset of action, simple inhalation, the avoidance of hepatic metabolism and improved bioavailability (this technology is applied to produce human insulin powder for inhalation, Afrezza ${ }^{\circledR}$ ). Early-stage clinical development for pain disorders is underway in the USA.

An inhaled formulation of CBD as a dry powder is also being developed by TFF Pharmaceuticals (USA) for the treatment of an undisclosed indication. The use of the Thin Film Freezing (TFF) platform [177], which is still in the research phase, yields 'Brittle Matrix Particles' that have low bulk density, high surface area and typically amorphous morphology, allowing the particles to supersaturate when in contact with lung tissue. Early research is underway in the USA [178, 179].

Molecular Infusions LLC (USA) has proposed a soft mist inhaler, with a formulation made up of cannabinoids in ethanol and glycerol solutions, to reduce pulmonary irritation [180].

\subsection{Transmucosal Administration}

Transmucosal drug administration indicates the absorption of a drug through the mucosal epithelium into systemic circulation. Intranasal, oral transmucosal and rectal routes are the major transmucosal routes that are used as alternatives to oral and parenteral administration.

A recent important review has thoroughly summarised the importance and limits of the transmucosal routes of drug administration in palliative care medication in order to 
provide suggestions to give the best possible care to patients at the end of life during the COVID-19 pandemic [181].

Oral transmucosal delivery is based on the direct uptake of the drug by the highly vascularised oral mucosa. Different pharmaceutical dosage forms have been developed, including orally disintegrating tablets, buccal mucoadhesive tablets, films and patches, sublingual disintegrating thin films, sprays, chewing gum and lozenges. Upon administration into the oral cavity, the formulation dissolves in a small amount of saliva and the released drug diffuses across the epithelial barrier, reaching systemic circulation and avoiding first-pass liver metabolism. Furthermore, better compliance and acceptance are expected, particularly for paediatric and geriatric patients, who often experience trouble in swallowing conventional tablets. One of the major challenges of buccal delivery is retaining the drug in the limited space in the mucosal oral cavity to achieve efficient partitioning. Furthermore, drugs can be unpalatable, bitter and can cause irritation to the oral mucosa, possibly leading to voluntary expulsion or swallowing. Physicochemical properties, such as small size (less than $500 \mathrm{Da}$ ), BCS class I or II, $\log P 2-4$ and high solubility in saliva, together with high pharmacological potency (meaning low doses are needed) are associated with achieving higher bioavailability. It is a popular route for drug administration that is used in palliative care, and several buccal formulations of opioid analgesics are commercially available.

A THC buccal formulation is being developed by IntelGenx Technologies Corp (CA) and Tetra-Bio Pharma for the treatment of cancer pain, chemotherapy-induced nausea, vomiting and anorexia and weight loss in people with AIDS. A rapid release, buccal tablet formulation of THC is being developed to provide immediate symptom relief and easier dose titration. The sublingual product is formulated using IntelGenx technology (AdVersa ${ }^{\mathrm{TM}}$ ), which is based on orally dissolving films that protect and solubilise the THC, thereby enhancing its stability and increasing its bioavailability [182].

Although several commercial products that contain CBD in oil have been proposed for transmucosal delivery, very few data are available on medicinal products in the literature and in patents. An oral sublingual formulation of CBD (BRCX 014) is being developed by Leaf Vertical Inc., now Diverse Biotech Inc. (USA), for the treatment of various cancers. Preclinical development is ongoing in the USA. The extraction and purification of CBD (>99\%) and some preliminary clinical tests, where cancer patients were treated with the standard-of-care therapy plus BRCX014, have been disclosed in a patent [183].

Moving on from CBD, Nabiximols formulations for use as an oromucosal spray (trade name Sativex ${ }^{\circledR}$ from GW Pharmaceuticals plc., UK) have been proposed for the treatment of different disorders. It has been launched in several countries for the treatment of muscle spasticity in patients with multiple sclerosis. In Canada, the product has been approved for the adjunctive treatment of cancer pain and neuropathic pain in adults with multiple sclerosis. Clinical development is underway for cancer pain, muscle spasticity, post-traumatic stress disorders and glioblastoma in multiple countries. In the USA, Nabiximols has FDA investigational new drug (IND) status for the treatment of spasticity. So far, 97 clinical trials have been carried out and 19 are either currently recruiting or planned. Nabiximols has also been proposed for use as an adjunctive analgesic treatment in adult patients with advanced cancer, who have moderate-to-severe pain during the highest tolerated dose of strong opioid therapy for persistent background pain [184]. Although not superior to placebo in terms of the primary efficacy endpoint, Nabiximols seems to provide multiple secondary endpoint benefits, particularly in patients with advanced cancer who receive a lower opioid dose, such as individuals with early intolerance to opioid therapy. Nevertheless, a recent systematic review and meta-analysis to identify randomised controlled trials of Nabiximols for the treatment of cancer-related pain was more critical [185]. In the six studies evaluated, when the change in pain intensity was the primary outcome, there was no statistically significant difference between the treatment and the placebo groups. Moreover, significant adverse effects and dropouts have been reported. One of the common adverse events of the chronic use of Nabiximols is mouth ulcerations (due to the ethanol excipient). An accurate evaluation to define the predictors of Sativex discontinuation in spasticity in multiple sclerosis has recently been published [186]. Physical disability and cognitive impairment were described for patients at risk of Nabiximols discontinuation over longterm follow-up. A Phase Ib trial of Nabiximols oromucosal spray and temozolomide in the treatment of patients with recurrent glioblastoma demonstrated acceptable safety and tolerability without drug-drug interaction (NCT01812603, NCT01812616) [187].

The proposed use of Nabiximols to treat chronic pain was recently reviewed by Uberall. Its analgesic efficacy was more apparent in placebo-controlled clinical trials of chronic neuropathic pain, and, in particular, those with multiple sclerosis-associated neuropathic pain, with some patients maintaining long-term (up to 2 years) benefits. A German Pain e-Registry analysis of patients with severe chronic pain treated in daily practice with self-administered Nabiximols showed the best results in the neuropathic pain subgroup, compared to the nociceptive or mixed pain subgroups [188]. When considering the well-being of patients with chronic pain and looking beyond pain phenomenology, the presence of anxiety and stress are important predictors of response to 
treatment. Both symptoms are reported to be significantly improved during treatment with the oromucosal spray.

The prevailing absorption route of oromucosal sprays is an interesting debate that may be, to some extent, correlated with results in pain treatment. Itin et al. have given a critical evaluation and proposed that the prevalent phenomenon was via the oral route, after swallowing, instead of absorption through the oral mucosa [189].

When selecting only Nabiximols trials with pain indications $(n=38)$, international clinical trials databases indicate that two studies are ongoing and are still recruiting; one on hyperalgesia in patients with deep endometriosis (EudraCT 2018-004378-92) and one involving patients with chronic kidney disease (EudraCT2019-002786-35). Regarding deep endometriosis pain, another Phase III study in the recruiting phase, is investigating the effects of sublingual CBD alone or in combination with norethindrone acetate (NCT04527003).

Another oro-buccal spray formulation of CBD and THC, which uses Medlab's patented medicine delivery system named NanoCelle ${ }^{\mathrm{TM}}$, is being developed by Aphria Inc (CA) and Medlab Clinical Ltd (AU), for the treatment of pain, including cancer pain. The technology enables submicron-sized particles to be taken as an oral spray. Clinical development for the treatment of cancer pain is underway in Australia [190, 191]. The preliminary results of a clinical trial $(n=25)$ (ACTRN12617001480370) have very recently shown that treatment with a CBD:THC (1:1) buccal spray resulted in an overall improvement in pain scores, including a greater than $30 \%$ average reduction in pain severity for the patient subgroup with bone metastasis $(n=8)$. Medlab have reported the development of a formulation of CBD and THC, named NanaBidial ${ }^{\mathrm{TM}}$, for the treatment of chemotherapy-induced nausea, vomiting and seizures.

Several oromucosal formulations (rapidly dissolving tablets) are in development. Pure Green Lab (USA) is studying rapidly dissolving sublingual tablets with cannabinoid combinations for the treatment of dysmenorrhea (measured by a pain scale score) in a Phase II study (NCT04091789). The formulation contains, as the active substances, 30 $\mathrm{mg}$ of CBD, $1 \mathrm{mg}$ THC, $97 \mathrm{mg}$ palmitoylethanolamide (which exerts a variety of biological effects, some related to chronic inflammation and pain) and a $0.2 \mathrm{mg}$ combination of myrcene, beta-caryophyllene, humulene, linalool and limonene. A recent paper proposed that palmitoylethanolamide has an interesting synergistic anti-nociceptive effect with cannabis extracts [192].

Pure Green Lab is also studying the use of sublingual dissolvable tablets, mainly containing CBD and THC $(1: 1)$, in a study on the pain associated with osteoarthritis of the knee (NCT04195269).

Ethicann Pharmaceuticals (CA) is developing a $\mathrm{CBD} /$ THC formulation using Catalent's well-known Zydis ${ }^{\circledR}$ oromucosal disintegrating tablet technology. These tablets disintegrate in the mouth in a very short time span, i.e., 20-30 s, and the therapeutic action of active agent originates upon contact with saliva. This tablet technology is reported to allow increased drug loads and better taste masking. It is a patented technology based on the principle of the freeze-drying process [193, 194]. The matrix is composed of polymers, such as gelatine, dextran, or alginates, that are able to form a glassy amorphous structure, plus saccharides and flocculating agents to prevent the sedimentation of water insoluble drugs. Permeation enhancers, such as surfactants, may also be added to optimise the transmucosal delivery of cannabinoids.

Oral and buccal formulations of cannabinoids have also been reported by NordicCan (DK) in different patents. These formulations include compressed gums, chewable liquid tablets, lozenges and pouch powder [195-197]. Analogously, Columbia Care LLC (USA) has filed a number of patents related to the delivery of cannabinoids via different routes; from sublingual/buccal formulations (of cannabinoid extracts with medium chain triglycerides, ethanol and polysorbate 80 as surfactant) to oral, topical and inhalation administration [198-201].

As the rectal mucosa is highly vascularised, this route of administration is useful for rapid systemic drug absorption; fast onset of action and a significant improvement in the bioavailability compared to the oral route (20-40\%) are observed [43]. Furthermore, suppositories are thought to be useful for the administration of cannabinoids in patient conditions that render swallowing impossible, and in conditions in which rectal administration is required, such as in situations experienced by patients as they approach the ends of their lives. Although no recent article has described this administration route for cannabinoids, a series of patents cover this topic. Axim Biotechnology has described the preparation of suppositories that are made up of cannabinoids in the form of a cannabis oil that is previously 'nano' encapsulated in a hyaluronic-derivative lattice [202].

A transmucosal cannabinoid formulation, including a chitosan excipient for rectal or vaginal delivery, has been reported by Tanaka [203]. The proposed method includes shea butter to extract cannabinoids from cannabis biomass, lecithin as a surfactant and the bioadhesive polysaccharide chitosan. This latter increased mucous-membrane and cellular permeability, while also increasing the adhesion of the product to the cavity mucous membranes.

Columbia Care LLC have also proposed suppositories made of a mouldable polymer that includes a mixture of PEG of various molecular weights (1450 and $4000 \mathrm{Da}$ ) and a concentration of $2 \%$ of cannabinoid extracts [204].

The intranasal route is particularly attractive for the management of acute pain and breakthrough pain in cancer patients $[205,206]$. Extensive vascularisation of the nasal 
mucosa facilitates the fast onset of action and non-invasive administration. Moreover, if the medication is in contact with the olfactory mucosa, it may bypass the blood-brain barrier and cross directly into the cerebrospinal fluid.

Bryson has described both semi-solid and liquid nasally administered cannabinoid compositions and a device to provide precise nasal administration. A range of different formulations (oils, emulsions, gels) were disclosed and a pharmacokinetic evaluation on a small group of healthy volunteers was performed [133]. Furthermore, in recent years, a series of patents has reported the nasal administration of cannabinoids, and have disclosed formulations and devices for administration, e.g., metered dose pump delivery devices as a multidose dispenser [207-211].

Nanostructured lipid carriers (NLCs), are made from a blend of solid and liquid lipids, in which the partially crystallised lipid particles, with mean radii $\leq 100 \mathrm{~nm}$, are dispersed in an aqueous phase containing one or more emulsifiers. NLCs provide an increase in the loading capacity of active agents in the nanoparticles and avoid leakage during storage. Although some works have described the potentiality of this carrier strategy, only a few works, which have exploited the mucoadhesive properties for nasal delivery, have recently been found [52, 212]. Matarazzo et al. have described the preparation, characterisation and in vivo nociceptive activity of NLCs loaded with CBD [52]. Three formulations were compared in vitro and in vivo: a $\mathrm{CBD}$ solution, CBD loaded in NLCs, and CBD loaded in NLC with the addition of an in-situ gelling polymer in the NLCs dispersion (Pluronic). This latter was proposed as a means to further increase the mucoadhesive properties. NLCs loaded with CBD were obtained using the hot microemulsion technique, which employed stearic acid, oleic acid and sorbitan monolaurate, while positively charged cetylpyridinium chloride was added to improve the bio-adhesiveness surface properties. The release studies showed that NLCs formulations exhibited a burst effect, releasing about $50 \%$ of their CBD within $5 \mathrm{~min}$. The in vivo evaluation in paclitaxel neuropathic induced pain showed that intranasally administered CBD-NLCs dispersion produced a more significant and lasting antinociceptive effect than the nasal administration of CBD, both as a solution and as an NLCs dispersed in the gel.

\subsection{Dermal and Transdermal Route}

Drug administration through the skin is facilitated by its easy accessibility. Dermal formulations can be divided into transdermal (for systemic effects) and topical (for local effects in the skin). From a physicochemical point of view, the requirements (recommendations) for transdermally administered drugs are: high potency (less than $10 \mathrm{mg} /$ day), small molecular weight (less than $500 \mathrm{~g} / \mathrm{mol}$ ), moderate lipophilicity $(\log P 1-5)$ and a melting point of less than $250{ }^{\circ} \mathrm{C}$.
To improve skin-layer permeability, compound "permeation enhancers" can be frequently added, although skin irritation is often produced [213].

More recently, procedures that are based on microneedles, thermal ablation and microderma abrasion have been investigated in human clinical trials to improve drug penetration and to also deliver macromolecules, therapeutic proteins, and vaccines.

Transdermal patches have been proposed for both acute and chronic pain management. There are various systems available for the delivery of non-steroidal anti-inflammatory drugs, opioids, local anaesthetics, capsaicin, and nitroglycerin. Patches are commonly used in paediatric practice.

Patches for the opioids fentanyl and buprenorphine are frequently used for symptom control and improvements to quality of life in patients with chronic pain related to osteoor rheumatoid arthritis, in the palliation of malignant pain and in the other persistent pain syndromes [214, 215].

Regarding neuropathic pain, pathology and symptoms are restricted to a localised area of the body for about $60 \%$ of patients. Several studies have shown that patients with localised or peripheral neuropathic pain respond to analgesic treatment with peripheral agents. [216, 217].

Considering the physicochemical properties of cannabinoids, evidently high lipophilicity ( $\log P$ around 6, against fentanyl at 3.9 and buprenorphine at 3.8) narrows skin permeability. Nevertheless, a skin reservoir effect has been observed for cannabinoids, and this is principally due to their highly lipophilic nature, which makes it easier for them to cross the stratum corneum but makes it difficult for them to traverse the aqueous dermis. Thus, synthetic approaches to produce prodrugs that are more skin permeable than the parent have been proposed by several companies. For an evaluation of the influence of vehicle-related aspects of CBD percutaneous absorption, the reader is directed to an article by Casiraghi et al. [218].

The case of Zynerba Pharmaceuticals Inc. (USA), who developed a synthetic D-glyceric acid ester pro-drug of THC administered as a transdermal patch (ZYN001 at $36 \mathrm{mg}$ dose), is indicative of the complexities inherent in properly delivering cannabinoids through the skin [219]. Zynerba conducted Phase I development in the treatment of fibromyalgia and neuropathic pain in Australia. However, development was subsequently discontinued (year 2018) because the study indicated that target blood levels of $5-15 \mathrm{ng} / \mathrm{mL}$ THC were not achieved.

An interesting nanotechnological approach is based on multi-layered lipid vesicles (MVLs), which were conceived to encapsulate THC for the topical and transdermal applications of cannabinoids in the treatment of pain. MVLs are formed by lipid bilayers that entrap both aqueous and oil phases in the form of a stabilised emulsion. Being lipophilic, cannabinoids are entrapped in the oil phase of the submicron 
emulsion and may be further enclosed between the phospholipid bilayers [220]. This formulation was proposed for transmucosal pain treatment by Altum Pharmaceuticals Inc (CA).

There is now increasing interest in transdermal applications of CBD. Preclinical data on the pharmacokinetics of CBD patches have been reported by Paudel et al. [221]. Transcutol-HP (diethylene glycol monoethyl ether), at $6 \%$ in a gel formulation, enhanced skin absorption 3.7-fold, in comparison with CBD in a propylene glycol-hydroxyethyl cellulose gel [222]. Further approaches to improving cannabinoid transdermal penetration have been proposed by Stinchcomb et al. who first studied the in vitro human skin transdermal flux of $\alpha 8$-THC, CBN and CBD [223]. The same authors, from AllTranz Inc. (USA), now called Zynerba Pharmaceutics, have studied parent drug labile derivatives, CBD-esters and -carbonates among others, that can permeate the stratum corneum and, once they cross it, quickly separate back into the parent drug [224, 225].

Transdermal CBD, using an hydroalcoholic gel, for the reduction of inflammation and pain in a rat, induced monoarthritis knee joint model was later studied [226]. This study confirmed that the transdermal administration of CBD may have a long-lasting therapeutic effect. A permeationenhanced CBD gel (Zygel ${ }^{\mathrm{TM}}$ ) for transdermal application then reached Phase II trials. Ethanol and propylene glycol were used as the solubilising agents, in addition to Transcutol-HP, which was used as the permeation enhancer. The alcohol content was about 54\% (w/w), while the CBD concentration was $4.2 \%(\mathrm{w} / \mathrm{w})$ in the final formulation. A recent patent claimed that this CBD gel could be used for the treatment of focal epilepsy [227]. Heussler has reported that the gel was well tolerated and produced clinically meaningful reductions in anxiety and behavioural symptoms in children and adolescents with fragile $X$ syndrome $(n=20)$, with an administration of $250 \mathrm{mg}$ of CBD daily as a gel $(n=18$, CBD administered up to 12 weeks) [228, 229], inducing a significant improvement in quality of life (NCT03614663). Clinical trials are still ongoing (ACTRN12618001063202 and NCT03802799). Other applications of Zygel for the treatment of epilepsy, developmental and epileptic encephalopathy, fragile-X syndrome, autism spectrum disorder (autistic disorders and pervasive child development disorders), DiGeorge syndrome and osteoarthritis have been reported in recent patents [227, 230-232].

A topical CBD gel, designated CBD 100, is being developed by Futura Medical (UK) for the treatment of pain, and, potentially, other indications. The therapy is being developed with the DermaSys ${ }^{\circledR}$ technology platform, which allows the rapid and targeted delivery of drug ingredients to be performed at the required therapeutic levels. Preclinical development is underway in UK.
Information on its web site indicates that Greene Street Pharma LLC (US) is developing a CBD transdermal patch for pain (still in research phase). The company is utilising its CBD-Cubed ${ }^{\mathrm{TM}}$ technology, which provides the sustainedrelease (24-h delivery) transdermal delivery of CBD via a proprietary platform that combines adhesive and enhancer technologies. Transdermal patches have many advantages over alternative routes of administration and have been shown to improve patient adherence, as well as maintaining a steady concentration in the bloodstream. Early research is underway in the US, but no further information is available on the principal databases.

Synthetic versions of CBD are being used by Botanix Pharmaceuticals (AU), via their dermal/transdermal drug delivery technology, Permetrex ${ }^{\mathrm{TM}}$, for the treatment of skin disorders, including acne, plaque psoriasis, rosacea, atopic dermatitis and bacterial skin diseases [233]. Formulations include hexamethyldisiloxane, which acts as a volatile silicone carrier fluid and filming component, the lubricant polypropylene glycol and oleyl alcohol, with CBD being dissolved in isopropyl alcohol to reach a concentration ranging from 5 and 20\%. Gel and spray formulations were proposed and clinical trials that investigated a topical occlusive system (BTX 1308) (ACTRN12618001802291) and a pump system (BTX 1503) (ACTRN12618001047280) were carried out. The exploration of novel silicone technologies in topical and transdermal drug delivery is quite important, as can be seen in the literature and filed patents [234, 235].

Topical cannabinoid delivery has been proposed for several skin-related therapeutic conditions (in the treatment of psoriasis, atopic dermatitis and acne) and has recently been reviewed by Sheriff et al. [236]. A range of topical CBD products are currently being marketed in the USA, Europe, Canada and Australia under OTC regulations.

In particular, the benefits that CBD provided as an antiinflammatory agent in three patients affected by epidermolysis bullosa have been described [237]. Paediatric patients benefited from the use of topical CBD (applied as an oil, cream, and spray by their parents) leading to a reduction in pain and blistering, as well as rapid wound healing.

Cannabinol has been observed to upregulate the expression of keratin 15, which improves skin integrity and reduces blister formation in patients with mutations of another keratin (keratin 14). Furthermore, CBN, which preferentially binds to higher affinity $\mathrm{CB} 2$ receptor, can inhibit the production of a variety of cytokines, and thus exhibit anti-inflammatory activity [238, 239].

A specific patent has also been filed by InMed Pharmaceutical Inc. (CA) [240], that described a cannabinol topical cream, designated INM-755, for the treatment of the symptoms of epidermolysis bullosa simplex, which is in development. A randomised, Phase I trial that examined the safety and tolerability of two strengths of INM-755 cream on intact 
skin in 22 healthy adult volunteers over a 14-day treatment period has recently been carried out (NCT04908215).

Cannabinoids are set to be clinically investigated for use in wound healing, although only anecdotal reports are currently available. Wound healing is an intricate process that includes overlapping inflammation, proliferation, and maturation/tissue-remodelling phases. Maida et al. have described topical cannabis-based medicines, applied to both wound beds and peri-wound tissues, as promising non-invasive and safe treatment options for non-uremic calciphylaxis leg ulcers [241]. A base gel carrier of hyaluronic acid was used to topically administer a mixture of CBD and THC, supplemented with known active flavonoids, including quercetin, diosmin, hesperidin and beta caryophyllene, to wounds.

Clinically significant wound-related-pain relief has been provided by medical cannabis oils containing both THC and CBD in a small series of cases involving malignant wounds [242], and pyoderma gangrenosum [243]. In all of the above-reported cases, analgesia, with opioid sparing, and a trend towards wound healing were all demonstrated. It was affirmed that the effect of cannabinoids may be beneficial in healing chronic wounds that are subject to prolonged inflammation.

It should be noted that One World cannabis (Subsidiary of OWC Pharmaceutical Research) completed a Phase I trial in healthy volunteers (NCT02976779) on the safety and tolerability of cannabis-extract-containing topical cream. A topical cream containing 3\% (CBD:THC) has also been described for the treatment of psoriasis, and early-stage clinical development is underway in Israel [244].

\subsection{Ophthalmic Delivery}

There is an evident, growing body of literature on the ophthalmic impact of cannabinoids and, in particular, on its potential intraocular-pressure-lowering effects, the beneficial effects on the retina, cornea and ocular motility. Current evidence on the effects of cannabis and related compounds on the disorders of the eye have recently been reviewed by Wang et al. [245]. The majority of clinical studies are related to cannabis consumption and ophthalmic effects, but preclinical evaluations of topical administration and potential therapeutic uses are increasing in number.

Although cannabinoids have demonstrated ocular hypotensive and neuroprotective properties, their use in treating glaucoma is limited by a significant number of potential side effects and by their limited solubility and penetration into ocular layers. Formulation studies and those on the synthesis of cannabinoid derivatives have flourished in recent years, although only preclinical evidence has been reported.

Improved formulations, based on cyclodextrins, topical solid lipid nanoparticles and fast gelling film formulations have also been reported. Some recent research on pain-treatment results is discussed below.

A preclinical study has investigated the antinociceptive and anti-inflammatory effects of THC, CBD and derivative HU-308 using an experimental mouse model of corneal hyperalgesia that was generated by chemical cauterisation [246] (Fig. 2). The topical application of THC, CBD, and HU-308 [247], dissolved in soybean oil, either as sole agents or in combination, resulted in a significant reduction in pain scores [248]. Tetra Bio Pharma is developing HU-308, in Phase I, for dry eyes and uveitis and, as a parenteral formulation, for the potential treatment of cytokine release syndrome and the potential prevention and treatment of SARS-COV-2 acute respiratory disease.

An approach to help treat diabetic retinopathy-induced pain and inflammation using the anti-inflammatory properties of CBD has been described by Taskar et al. In order to produce a therapeutic effect, the molecule has to transverse the static ocular layers and the dynamic blood-ocular barriers to reach the back of the eye. CBD analogues with amino-acid and dicarboxylic-acid ligands were synthesised to improve solubility. Figure 2 reports mono-substituted valine hemi-succinate, which significantly enhanced ocular bioavailability after topical application, compared to the other studied derivatives. All of the analogues were formulated into a soybean oil-based emulsion using block copolymer Pluronic F68 (Tocrisolve) as the emulsifier; a preclinical evaluation on rabbits was also reported [249].

InMed Pharma Inc. (USA) is actively working on cannabinoid eye delivery. For example, a hydrogel composite of the biocompatible and highly mucoadhesive polymers hyaluronic acid and methyl-cellulose has been used to deliver cannabigerolic acid. In order to achieve sustained release for a prolonged duration, the drug was loaded into nanoparticles (composed of poly(ethylene oxide) and polylactic acid). An increase in in vitro corneal permeation of $300 \%$, over control formulations, was observed [250, 251]. Moreover, advanced formulations, made up of stimulus-responsive nanoparticle gels, for glaucoma treatment have also been proposed [252].

Solid lipid nanoparticles (SLNs) are submicron particles made from solid lipids that have the potential to deliver hydrophobic compounds, such as cannabinoids, in different treatment settings. SLNs have proven themselves to be suitable carriers for lipophilic substances and overcome hurdles in treating the eye posterior segment [253]. According to this approach, SLNs of THC-valine-hemi-succinate (THC-VHS) (a hydrophilic prodrug of THC with a better permeation profile than THC) were investigated, along with a nanoemulsion of THC-VHS, as a means to lower intraocular pressure in normotensive rabbits.

SLNs have been prepared using glyceryl behenate as the lipid phase, while the aqueous phase comprised polyoxyethylene-polyoxypropylene block copolymer (Pluronic F-68) 
and polysorbate 80 as surfactants, plus glycerin. The interest in SLNs lies in their ability to prolong the residence time of the formulation in the cul de sac, thus acting as a drug reservoir and enhancing ocular bioavailability. Results have showed that THC-VHSSLNs, with multiple dosing, were able to achieve an intense and long-lasting intraocular pressure drop, compared to the nanoemulsion formulation [254].

SLN can be also cited as a co-encapsulation approach, using dexanabinol (a synthetic cannabinoid derivative with neuroprotective effects) and curcumin (exhibiting antidepressant effects in the treatment of major depressive disorder) to evaluate the antidepressant effects in a mouse model. The study revealed that, although SLNs are able to overcome instability and the poor absorption of the loaded molecules, they had no antidepressant effects on the $\mathrm{CBR} 1^{-/-}$mouse models of major depressive disorder [255].

\subsection{Less-Explored Delivery Routes for Cannabinoids and Other Preliminary Studies}

Because the parenteral administration of cannabinoids is not a common route, formulation studies are rare. Nevertheless, two recent studies on human subjects can be cited. The first is related to the characterisation of the pharmacokinetics of THC and its major metabolites in healthy volunteers, with known CYP2C9 status, using non-compartmental analyses, compartmental modelling and minimal physiologically based pharmacokinetic modelling and was performed by Wolowich et al. [256].

Another recent exploratory, randomised, double-blind, placebo-controlled, cross-over study tested the effects of the IV administration of THC on pain response using a variety of human experimental pain models (intradermal capsaicin, mechanical, electrical, heat and cold procedures) [257] (NCT01595620). The conclusions of this investigation reveal that THC lacks antinociceptive effects in several forms of experimentally induced acute pain. The authors concluded that additional factors, such as pain paradigm, mode of drug administration, timing and other constituents of cannabis may be relevant and worth exploring. At the same time, there is a need for larger, high-quality, controlled studies to answer the growing demand for clinical applications of cannabinoids for pain management.

Nutrae LLC (USA) has filed three patents on the micellar encapsulation of cannabinoids for different administration routes (oral, IV, or inhalations and transdermal) [258-260].

Of the wide range of patents filed by GW Pharmaceutics, two report non-oily formulations for parenteral administration [261,262]. The surfactant that is able to improve the solubility of CBD, in order to reach a water injectable solution $3-5 \mathrm{mg} / \mathrm{mL}$, was macrogol 15 -hydroxystearate (e.g., Solutol HS15), while glycerol and glucose appeared to be the best isotonic agents. Stability enhancers have also been added.

Several micro and nanotechnological applications to parenteral administration of cannabinoids are in development, although in preclinical trials. The most innovative examples are described below.

In an industrial project in development, Cardiol Therapeutics has developed a polymeric micellar formulation of CBD (CTX 01) for subcutaneous administration. This was designed to optimise and maintain blood levels of antiinflammatory and antifibrotic drugs and target their action to sites of inflammation and fibrosis for the treatment of heart failure. A CBD-loading value of $12 \%$ in micelles, with sizes of less than $100 \mathrm{~nm}$, was obtained using a block copolymer with a structure consisting of methoxy poly(ethylene oxide)block-poly ( $\alpha$-benzyl carboxylate- $\varepsilon$-caprolactone). Pharmacokinetic evaluations in rats showed that micellar CBD (with $4 \%$ CBD loading), subcutaneously administered, led to a greater amount of drug entering the bloodstream over 72 $h$ than free CBD solubilised in PEG 300 administered by same route [263]. The micelles seemed to preferentially and passively, localise in fibrotic tissue when administered systemically or subcutaneously, and more preferentially in association with cardiac fibroblasts.

Another nanotechnology formulation that is based on polymer loading CBD has been proposed by the Aphios Corporation (USA) as a product (compounds APH-1501 and APH-1403). CBD was encapsulated into Poly Lacticco-Glycolic Acid (PLGA) polymer nanospheres using a superfluid stream of $\mathrm{CO}_{2}$ and ethanol co-solvent, and then decompressed into a $1 \%$ polyvinyl alcohol buffered solution [264].

Another patented approach for the formulation of cannabinoid forms that are suitable for ingestion and topical application makes use of a cannabinoid-containing micellar aqueous solution of poloxamer and tocopherol-like compounds. The cannabinoids are thus stabilised against oxidation and/or photochemical degradation (SolMic Research $\mathrm{GmbH}, \mathrm{DE}$ ) [265].

Melt-printed polymeric micro-depots for full-spectrum CBD-rich extract administration have been proposed as long-acting formulations to release the whole-plant cannabis extract instead of its individual components. The formulation consisted of polycaprolactone microspheres that were able to significantly extend the circulation time of multiple phytocannabinoids, which have been suggested to have superior therapeutic effects compared to individual components. Moreover, the long-term anticonvulsive activity of the micro-depots was observed after subcutaneous administration in a pentylenetetrazol-induced convulsion mouse model [266].

Lipid nanocapsules (LNCs) are biocompatible and biodegradable carriers that contain an inner oily core and are 
prepared using a solvent-free and up-scalable process that is based on the energy-efficient phase inversion temperature method [267]. LNCs may be a suitable brain drug-delivery vehicle, while surface modification can further improve bioavailability towards the central nervous system. For example, LNCs have been loaded with CBD using a mixture of caprylic-capric acid triglycerides, as the oily vehicle, and poly-oxyethylated 12-hydroxystearic acid and soybean lecithin as the surfactants. The choice of the oily phase was based on the solubility of CBD, which can lead to a CBD/ oil (w/w) loading of $15 \%$. The comparison between two differently sized LNCs (20- and 50-nm) showed that the $\mathrm{IC}_{50}$ value was lower for smaller LNCs, as a consequence of the modified CBD release [268]. Furthermore, the surface functionalisation of LNCs with CBD allowed the cannabinoid receptors overexpressed in glioma cells to be targeted, as demonstrated in permeability experiments across an in vitro blood-brain barrier model and in biodistribution experiments in mice [269]. Finally, the combination of CBD-loading and CBD-targeting has been observed to further reduce the $\mathrm{IC}_{50}$ values in glioma cells [268].

Other application of cannabinoids as potential anticancer agents regarded the use of micro and nanopolymeric particles for parenteral administration [270]. CBD was loaded into PLGA microparticles and compared to CBD in solution when administered in combination with either paclitaxel or doxorubicin for the evaluation of anticancer activity on breast cancer cells. Results showed that CBD-microparticles were effective as a monotherapy, showing extended anti-proliferative activity, and also when combined with paclitaxel or doxorubicin. The anticancer efficacy of CBD-microparticles as a monotherapy and in combination with paclitaxel in triple negative breast tumours was also confirmed in the chick chorioallantoic membrane model [271].

Solid nanoparticles can be also produced applying a bottom-up method based on antisolvent precipitation. This is a facile method with which to achieve the precipitation and stabilisation of nanoparticles of poorly water-soluble drugs [272]. CBD nanosuspensions have been prepared using this method with the addition of surfactants as stabilisers. In particular, different parameters were tuned in the formulation, such as the concentration of the mixture Span 80/Tween $80(1: 4 \mathrm{w} / \mathrm{w})$, CBD concentration and solvent/water ratio with ethanol as a solvent. The presence of surfactants stabilised the mean dimensions of the formulations over several months of storage [273].

\section{Discussion}

The previous sections describe the efforts and interests of company research that has several different cannabinoids formulations in the pipeline, while numerous products are in advanced studies for a series of clinical needs. Table 3 summarises ongoing clinical trials on the potential efficacy and clinical advantages of cannabinoids in the treatment of different pain conditions. The most frequent formulations are based on pure THC and standardised combinations of THC:CBD, while the preferred administration route is oral, followed by inhalation and transmucosal sublingual.

In parallel with the continuation of clinical evaluations, several systematic meta-analyses, reviews and novel clinical evaluations on the efficacy and safety of different cannabinoids in different formulations via different administration routes have very recently been reported.

Selecting studies focused on acute pain, Gazendam has analysed six oral administration trials, mainly, of THC. This analysis revealed that there was a small, but significant, reduction in subjective pain scores compared with placebo in patients experiencing acute pain [274].

More comparative evaluations have been reported for chronic non-cancer pain treatments.

Johal et al. have selected and evaluated 36 trials and found that cannabinoids are an effective form of pain control in this patient population, with a particularly strong effect for cannabinoids, mainly THC, that are orally administered, rather than oromucosally administered or smoked. There was moderate-quality evidence for cannabinoids having a small effect in treating chronic, non-cancer-related pain at all time points studied, up to 6 months. Moreover, small effects in pain reduction were found in patients with neuropathic pain related to multiple sclerosis [275]. Furthermore, another recent evaluation demonstrated that Bedrocan with $22 \%$ THC, administered with the Syqe Inhaler to 27 patients with neuropathic pain/complex-regional pain syndrome, provided a statistically significant decline in pain intensity, measured fifteen minutes after the inhalation of 0.5 and 1.0 $\mathrm{mg}$ THC doses, and beyond [276]. Although the NIC guidance on cannabis-based medicinal products allows for their prescription when there is an unmet clinical need related to chronic pain, $\mathrm{CBD}$, alone or combination with $\mathrm{THC}$, is not suggested for administration unless part of clinical trials [277].

Considering medical cannabis for the reduction of opioid dosage in the treatment of non-cancer chronic pain, nine studies have been performed in which cannabis was smoked at a very wide range of concentrations $(1.5-2000 \mathrm{mg})$. The nine available studies suggested that cannabis was effective, as an adjunct to opioids, in reducing the dosage of opioids in study participants. However, the design of several studies provides a limited basis upon which to make a rational, evidence-based recommendation [278].

Barry and Chris have presented a retrospective cohort study, from 2014-2018, in British Columbia. The outcomes of interest included the use of opioid/non-opioid pharmacotherapy, the number/frequency of visits and the proportion 
of patients able to reduce/discontinue opioid therapy. The non-opioid pharmacotherapy with the highest proportion of patients perceiving a benefit was nabilone at $78 \%$, while the lowest proportion was with gabapentinoids at $52 \%$. Seventy patients with different chronic non-cancer pain disorders on opioid therapy demonstrated that almost $90 \%$ were able to successfully taper their opioid use, with an approximate $60 \%$ mean dose reduction over 12 months [279].

The available body of literature suggests that medical cannabis may be effective for the treatment of various noncancer-related pain disorders.

Nabiximols has undergone larger and more robust clinical trials than other cannabis-based products or medication for use in pain [280]. Nevertheless, head-to-head trials comparing the safety and efficacy of types of medical cannabis are still expected.

Indeed, Mohiuddin et al. have focused their systematic review on the quality of safety assessment and reporting in chronic non-cancer pain cannabinoid trials $(n=43)$. When assessing the specific adverse effects experienced by trial participants, there were numerous effects that could adversely impact quality of life and adherence to treatment. Across all intervention subtypes, the most frequent adverse effects included dizziness, headache, fatigue, drowsiness, nausea, dry mouth, muscular weakness, headache and euphoria [281]. The frequency of serious adverse effects was $19.1 \%$ in studies of dronabinol, $4.4 \%$ in studies of oral THC:CBD capsules, $3.3 \%$ in studies of Nabiximols and $0 \%$ in studies of smoked THC and nabilone.

A randomised, double-blind, placebo-controlled trial on treating fibromyalgia with cannabinoids has recently been presented by Chaves et al. [282]. Oil extracts of cannabis had $24 \mathrm{mg} / \mathrm{mL}$ THC and $0.5 \mathrm{mg} / \mathrm{mL}$ CBD, although the terpenoid profile was not analysed, were administered sublingually to 18 patients. The mean dose used in this study was 3.6 drops a day ( $4.4 \mathrm{mg}$ of THC and $0.08 \mathrm{mg}$ of CBD). At each visit, patients filled out a validated self-administered test that evaluated physical function, work status, well-being and associated physical and mental symptoms. Statistically significant improvements in the "feel good," "do work," and "pain" items in the cannabis group were found upon an analysis of the groups, and an additional improvement in the "fatigue" item was found in this same group, compared with the baseline value. However, the main cause of this benefit remains unclear.

A systematic review and meta-analysis on cancer-related pain management has identified few studies (six included) [185]. The Sativex oromucosal spray was administered versus placebo, and, in some studies, patients self-titrated to the optimal dose or were randomly assigned to different doses. Where change in pain intensity was the primary outcome, there was no difference between cannabinoids and placebo in the change in average numeric rating pain scale scores.
An evaluation of the six randomised, double-blind, placebocontrolled clinical trials where Nabiximols was administered for the treatment of pain in patients with cancer has shown that the differences in the studies may be caused by the varying study designs. However, the dosing of Nabiximols also varied among the studies. Some of the studies allowed selftitration, whereas others provided a specific dose and dosing interval. At this time, data supporting the effectiveness of cannabis and cannabinoids in the treatment of cancerrelated pain are limited. The conclusion of the authors is that Nabiximols may be an effective addition to cancer pain treatment in those who are not optimised on opioid therapy, but that effectiveness varies widely from one patient to the next. Thus, it may be proposed as being more beneficial as an adjunct to the standard-of-care treatment rather than as a replacement [283]. Moreover, Hauser has analyzed five studies and affirmed that there was no evidence-based argument to prescribe the use of medical cannabis or nabilone in cancer patients, but it was confirmed that more patients reported pain relief of $30 \%$ or greater in the Nabiximols arm than in the THC arm (23\%) [284]. Nevertheless, cannabinoids have been also proposed for treatment of neuropathic pain induced by chemotherapeutics such as platinum derivatives or taxanes [285, 286]. This pain can be intense and poorly controlled by the use of opioids and the administration of THC in these patients, in addition to current pain therapy, may reduce the required dose of opioid, inducing a sense of well-being, thus counteracting the depression often present in the cancer patient. In addition, cannabinoids could improve the quality of sleep, having an anxiolytic effect.

There have been a number of studies that have focused not only on pain, but also on HRQoL. A recent study provided 12 months' follow-up and has shown how the long-term use of cannabis can reduce pain intensity and pain-related interference scores. Moreover, HRQoL and general healthsymptom scores were improved compared with baseline, above all in men. Furthermore, the authors pointed out that patients, and even physicians, preferred cannabis in the form of oil. The favourable response to oil-based cannabis may be due to the slower onset of the effect compared with smoking cannabis, as well as easier dose titration. Despite this interesting result, the authors highlighted that depression scores increased during the study [287]. Although the results are not conclusive, it is important to underline that further studies have shown that cannabis may have negative effects on the mental health of treated patients [288, 289], and that it may exacerbate neurological diseases such as epilepsy and anxiety [289].

It has also been highlighted that cannabis use has been associated with lower HRQoL in cancer patients. Nevertheless, patients would continue to use it, above all if severe symptoms are present. The effect of cannabis on cancer symptoms may be related to the amount of THC and its 
ratio with $\mathrm{CBD}$ in the administered formulation, as well as to the dosage and method of intake [290].

Previous intake for medical or recreational purposes is a crucial aspect to consider when evaluating the effect of cannabis. This is in order to better understand the real perception of the effects in patients, who may be "naïve" or otherwise [291, 292].

Patients often report subjective benefits from cannabis for which there is no scientific evidence. It then becomes crucial to understand whether the subjective perceptions of cannabis benefits may be related to significant and persistent changes in subjective well-being that may be scientifically measurable.

Several studies have reported HRQoL evaluations for patients affected by pain and other pathologies that have been treated with cannabis, but, unfortunately, the majority of these studies have limitations and cannot therefore be considered conclusive. First, the applied evaluation methods were not always uniform. In addition, the wellbeing evaluation was often not a primary outcome, and the duration of the studies and sample sizes were highly variable [25]. Above all, the prescribed types of cannabis were different, both in terms of the variety, and therefore in the quantities of administered active molecules, and the route and time of administration. These aspects make the studies difficult to compare with each other [283].

The interesting results of a clinical audit on prescribed CBD oil have recently been described by Gulbransen et al. [293]. The study included 400 patients that were studied by Cannabis Care, New Zealand. The outcomes were defined using descriptive system measures of five domains (mobility, self-care, usual activities, pain or discomfort, anxiety, or depression), each with five levels of severity. The analgesic and anxiolytic benefits of CBD were observed in patients with non-cancer chronic pain and mental health conditions, such as anxiety. CBD was well tolerated, making it safe to trial for non-cancer chronic pain, mental health, neurological and cancer symptoms.

By analysing the administration route, cannabinoids used and formulations, a summative meta-analysis of cannabinoids for pain control has revealed that THC/CBD and THC, administered via the oromucosal route, represent the best score for neuropathic and cancer pains, while THC via the oral route had the best score for cancer pain, standardised dried cannabis via the inhalation route was best for neuropathic pain, and cannabis extract via the oral route was best for nociceptive pain. This suggests that pain reduction depends on the type of cannabis or cannabinoids used, the administration route and the type of pain [294]. CBD alone, via different routes and in different formulations, seemed not to play a role in pain reduction. Nevertheless, a more recent preclinical study on different cultivars, which were extracted and well characterised, showed a similar analgesic effect both when the main component was THC and CBD, but, in this last case, the adverse effect (depression of respiratory rate) was not present [295].

The evidence for transdermal applications is increasing, and more effort to improve the efficacy and use of this route, which has shown 'drug abuse' potential, would be welcome. This route of adsorption has appeared to be relevant for several companies in recent years, and, as an example, a CBD gel was well tolerated and produced clinically significant results in fragile $\mathrm{X}$ syndrome. This approach could be further improved by exploiting the well-known microneedle system, in which biodegradable polymers deliver fluid material by crossing biological barriers [296].

Among the oral formulations that stand out, we can find some novel approaches including Accordion Pills and cocrystallisation, while the molecular conjugation approach (Kalytera) is more challenging. Moreover, cannabinoid formulations can take advantage of modern printing technologies, such as the fabrication of data-enriched edible pharmaceuticals (DEEP), which contain lipophilic cannabinoids (THC and CBD) that are inkjet-printed onto a porous substrate in the pattern of a QR code. These oral forms may allow the preparation of personalised doses and, at the same time, the incorporation of relevant data that are useful for the traceability of the drug product at a single dosage unit level. In this way, the strategy changes from "drug package labelling" into "in-drug labelling" [297].

Furthermore, transmucosal wafers have also been explored to improve the bioavailability of CBD [298]. Wafers are a simple drug carrier matrix with amorphous holes that can encapsulate oily CBD and facilitate sublingual delivery. A very recent clinical trial was conducted in Australia and involved the sublingual administration of an oil and wafer drug-carrier matrix containing an oily solution with a high CBD content (Linnea 315CSE extract). The oily solutions and wafer were well tolerated and achieved increased CBD plasma concentrations compared to an available commercial Nabiximols formulation [299]. Transmucosal Nabiximols have provided important results, and other formulations, such as tablets with mucoadhesive properties to avoid swallowing and increase absorption, have been considered as a means to improve performance.

Furthermore, this administration route has been explored for the development of promising delivery systems that also use nanotechnology approaches [300].

Despite the number of studies concerning micro- and nano-formulations, a comparison of efficacy in delivering cannabinoids remains difficult. Indeed, the literature reports experimental settings with different carriers, loaded molecules (pure active pharmaceutical ingredients or extracts) and in vitro/in vivo tests. Although interesting results in animal models have been described, the pharmacokinetics have proven to be model-dependent, not uniform among the 
research groups, and affected by the vehicle of administration. Moreover, the intended target pathology is sometimes lacking in the papers. As an example, only one patent has reported an in vivo test on dogs to evaluate the management of neuropathic pain in the time period considered in this review [151]. Micro- and nano-formulation studies therefore appear to be very preliminary attempts to explore the possibility of exploiting nanotechnologies to deliver cannabinoids. From this point of view, lipid carriers seem to be the most suitable vehicle for lipophilic cannabinoids. Furthermore, their production processes are scalable, although a complete physico-chemical characterisation has still to be carried out in order to standardise the described approaches.

Moreover, very few new nano-formulations are currently on the market or under clinical evaluation. This is probably because the most used cannabinoid-administration routes (i.e., oral and transmucosal) do not require the encapsulation of cannabis in nanosystems to prepare suitable formulations such as capsules or tablets.

\section{Conclusion}

In conclusion, the examples reported in the present review indicate that many remarkable formulations involving cannabinoids are now evolving and are being validated in several clinical trials. Nevertheless, there is an urgent need for further research to investigate the optimal route and composition of cannabinoids in pain treatment, in large, highquality randomised clinical trials that can shed light onto the risks and benefits of cannabinoids in a controlled patient population. Multidisciplinary efforts, involving pharmacological and pharmaceutical technological sciences, have been joined by increased investment in research to help those who suffer, while not unintentionally causing hazardous consequences. At the same time, the legal, ethical, and medical aspects of improving regulation will be crucial if high quality, high-safety cannabinoid medicines are to be produced.

Acknowledgements The work was partially funded by Italian Ministry of Education, Universities and Research-University of Torino "Fondi Ricerca Locale (ex-60\%)". Dale James Matthew Lawson is gratefully thanked for correcting English of the manuscript.

\section{Declarations}

Funding Open access funding provided by Università degli Studi di Torino within the CRUI-CARE Agreement. The work was partially funded by Italian Ministry of Education, Universities and ResearchUniversity of Torino.

Author contributions BS performed the literature search, wrote, reviewed, and edited the manuscript. FB performed the literature search, reviewed the data, and edited the manuscript. CDP wrote, reviewed, and edited the manuscript. SA reviewed data and reviewed and edited the manuscript. DG reviewed data and edited the manuscript. FD performed the literature search, reviewed data, wrote, reviewed, and edited the manuscript.

Conflict of interest Barbara Stella, Francesca Baratta, Carlo Della Pepa, Silvia Arpicco, Daniela Gastaldi, Franco Dosio have no conflicts of interest that are directly relevant to the content of this article.

Ethics approval Not applicable.

Consent to participate Not applicable.

Consent for publication Not applicable.

Availability of data and material Not applicable.

Code availability Not applicable.

Open Access This article is licensed under a Creative Commons Attribution-NonCommercial 4.0 International License, which permits any non-commercial use, sharing, adaptation, distribution and reproduction in any medium or format, as long as you give appropriate credit to the original author(s) and the source, provide a link to the Creative Commons licence, and indicate if changes were made. The images or other third party material in this article are included in the article's Creative Commons licence, unless indicated otherwise in a credit line to the material. If material is not included in the article's Creative Commons licence and your intended use is not permitted by statutory regulation or exceeds the permitted use, you will need to obtain permission directly from the copyright holder. To view a copy of this licence, visit http://creativecommons.org/licenses/by-nc/4.0/.

\section{References}

1. Bar-Lev Schleider L, Abuhasira R, Novack V. Medical cannabis: aligning use to evidence-based medicine approach. Br J Clin Pharmacol. 2018;84(11):2458-62.

2. Loeser JD, Treede RD. The Kyoto protocol of IASP basic pain terminology. Pain. 2008;137(3):473-7.

3. Zelaya CE, Dahlhamer JM, Lucas JW, Connor EM. Chronic pain and high-impact chronic pain among US adults, 2019. NCHS Data Brief. 2020;390:1-8.

4. Abbafati C, Abbas KM, Abbasi-Kangevari M, Abd-Allah F, Abdelalim A, Abdollahi M, et al. Global burden of 369 diseases and injuries in 204 countries and territories, 1990-2019: a systematic analysis for the Global Burden of Disease Study 2019. Lancet. 2020;396(10258):1204-22.

5. O'Grady C. Cannabis research data reveals a focus on harms of the drug. Science. 2020;369(6508):1105.

6. Bruni N, Della Pepa C, Oliaro-Bosso S, Pessione E, Gastaldi D, Dosio F. Cannabinoid delivery systems for pain and inflammation treatment. Molecules. 2018;23(10):2478.

7. Patra JK, Das G, Fraceto LF, Campos EVR, Rodriguez-Torres MDP, Acosta-Torres LS, et al. Nano based drug delivery systems: recent developments and future prospects. J Nanobiotechnol. 2018;16(1):71.

8. Gureje O, Von Korff M, Simon GE, Gater R. Persistent pain and well-being: a World Health Organization study in primary care. J Am Med Assoc. 1998;280(2):147-51.

9. Fayaz A, Croft P, Langford RM, Donaldson LJ, Jones GT. Prevalence of chronic pain in the UK: a systematic 
review and meta-analysis of population studies. BMJ Open. 2016;6(6):e10364.

10. Burt CW, Schappert SM. Ambulatory care visits to physician offices, hospital outpatient departments, and emergency departments: United States, 1999-2000. Vital and Health Statistics, Series 13:2006(159):1-66. Centers for Disease Control and Prevention, National Center for Health Statistics Hyattsville, Maryland

11. Voon P, Karamouzian M, Kerr T. Chronic pain and opioid misuse: a review of reviews. Subst Abuse Treat Prev Policy. 2017;12(1):1-9.

12. Fitzcharles MA, Cohen SP, Clauw DJ, Littlejohn G, Usui C, Hauser W. Nociplastic pain: towards an understanding of prevalent pain conditions. Lancet. 2021;397(10289):2098-110.

13. Pacher P, Kogan NM, Mechoulam R. Beyond THC and endocannabinoids. Annu Rev Pharmacol Toxicol. 2020;60:637-59.

14. Cristino L, Bisogno T, Di Marzo V. Cannabinoids and the expanded endocannabinoid system in neurological disorders. Nat Rev Neurol. 2020;16(1):9-29.

15. Zou S, Kumar U. Cannabinoid receptors and the endocannabinoid system: signaling and function in the central nervous system. Int J Mol Sci. 2018;19(3):833.

16. Pagano C, Navarra G, Coppola L, Bifulco M, Laezza C. Molecular mechanism of cannabinoids in cancer progression. Int $\mathrm{J}$ Mol Sci. 2021;22(7):3680.

17. Malhotra P, Casari I, Falasca M. Therapeutic potential of cannabinoids in combination cancer therapy. Adv Biol Regul. 2021;79:100774.

18. Luís Â, Marcelino H, Rosa C, Domingues F, Pereira L, Cascalheira JF. The effects of cannabinoids on glioblastoma growth: a systematic review with meta-analysis of animal model studies. Eur J Pharmacol. 2020;876:173055.

19. Bethoux F, Marrie RA. A cross-sectional study of the impact of spasticity on daily activities in multiple sclerosis. Patient PatientCent Outcomes Res. 2016;9(6):537-46.

20. Nielsen S, Germanos R, Weier M, Pollard J, Degenhardt L, Hall $\mathrm{W}$, et al. The use of cannabis and cannabinoids in treating symptoms of multiple sclerosis: a systematic review of reviews. Curr Neurol Neurosci Rep. 2018;18(2):1-12.

21. García-Gutiérrez MS, Navarrete F, Gasparyan A, AustrichOlivares A, Sala F, Manzanares J. Cannabidiol: a potential new alternative for the treatment of anxiety, depression, and psychotic disorders. Biomolecules. 2020;10(11):1-34.

22. Aran A, Harel M, Cassuto H, Polyansky L, Schnapp A, Wattad $\mathrm{N}$, et al. Cannabinoid treatment for autism: a proof-of-concept randomized trial. Mol Autism. 2021;12(1):6.

23. Uddin SM, Al Mamun A, Sumsuzzman DM, Ashraf GM, Perveen A, Bungau SG, et al. Emerging promise of cannabinoids for the management of pain and associated neuropathological alterations in alzheimer's disease. Front Pharmacol. 2020;11:1097.

24. Raucci U, Pietrafusa N, Paolino MC, Di Nardo G, Villa MP, Pavone $\mathrm{P}$, et al. Cannabidiol treatment for refractory epilepsies in pediatrics. Front Pharmacol. 2020;11:586110.

25. Goldenberg M, Reid MW, IsHak WW, Danovitch I. The impact of cannabis and cannabinoids for medical conditions on healthrelated quality of life: a systematic review and meta-analysis. Drug Alcohol Depend. 2017;174:80-90.

26. Karimi M, Brazier J. Health, health-related quality of life, and quality of life: what is the difference? Pharmacoeconomics. 2016;34(7):645-9.

27. Ebrahim S. Clinical and public health perspectives and applications of health-related quality of life measurement. Soc Sci Med. 1995;41(10):1383-94.

28. Bouso JC, Jiménez-Garrido D, Ona G, Woźnica D, dos Santos RG, Hallak JEC, et al. Quality of life, mental health, personality and patterns of use in self-medicated cannabis users with chronic diseases: a 12-month longitudinal study. Phytother Res. 2020;34(7):1670-7.

29. Gruber SA, Sagar KA, Dahlgren MK, Gonenc A, Smith RT, Lambros AM, et al. The grass might be greener: medical marijuana patients exhibit altered brain activity and improved executive function after 3 months of treatment. Front Pharmacol. 2017;8:983.

30. Jett J, Stone E, Warren G, Cummings KM. Cannabis use, lung cancer, and related issues. J Thorac Oncol. 2018;13(4):480-7.

31. Liano HC, Zakowicz P, Mikołajczak P. Cannabinoids as antiemetics: a short review. Acta Poloniae Pharmaceutica Drug Res. 2018;75(5):1063-8.

32. Bar-Lev Schleider L, Mechoulam R, Lederman V, Hilou M, Lencovsky O, Betzalel O, et al. Prospective analysis of safety and efficacy of medical cannabis in large unselected population of patients with cancer. Eur J Intern Med. 2018;49:37-43.

33. Gotfried J, Naftali T, Schey R. Role of cannabis and its derivatives in gastrointestinal and hepatic disease. Gastroenterology. 2020;159(1):62-80.

34. Gottschling S, Ayonrinde O, Bhaskar A, Blockman M, D'Agnone O, Schecter D, et al. Safety considerations in cannabinoid-based medicine. Int J Gen Med. 2020;13:1317-33.

35. Russo EB. Taming THC: potential cannabis synergy and phytocannabinoid-terpenoid entourage effects. Br J Pharmacol. 2011;163(7):1344-64.

36. ElSohly MA, Slade D. Chemical constituents of marijuana: the complex mixture of natural cannabinoids. Life Sci. 2005;78(5):539-48.

37. Stone NL, Murphy AJ, England TJ, O’Sullivan SE. A systematic review of minor phytocannabinoids with promising neuroprotective potential. Br J Pharmacol. 2020;177(19):4330-52.

38. Booth JK, Bohlmann J. Terpenes in Cannabis sativa-from plant genome to humans. Plant Sci. 2019;284:67-72.

39. Benet LZ, Broccatelli F, Oprea TI. BDDCS applied to over 900 drugs. AAPS J. 2011;13(4):519-47.

40. Perucca E, Bialer M. Critical aspects affecting cannabidiol oral bioavailability and metabolic elimination, and related clinical implications. CNS Drugs. 2020;34(8):795-800.

41. Franco V, Gershkovich P, Perucca E, Bialer M. The interplay between liver first-pass effect and lymphatic absorption of cannabidiol and its implications for cannabidiol oral formulations. Clin Pharmacokinet. 2020;59(12):1493-500.

42. Lucas CJ, Galettis P, Schneider J. The pharmacokinetics and the pharmacodynamics of cannabinoids. Br J Clin Pharmacol. 2018;84(11):2477-82.

43. Grotenhermen F. Pharmacokinetics and pharmacodynamics of cannabinoids. Clin Pharmacokinet. 2003;42(4):327-60.

44. Romano L, Hazekamp A. An overview of galenic preparation methods for medicinal cannabis. Curr Bioact Compd. 2019;15(2):174-95.

45. Schlag AK. An evaluation of regulatory regimes of medical cannabis: what lessons can be learned for the UK? Med Cannabis Cannabinoids. 2020;3(1):76-83.

46. Baratta F, Simiele M, Pignata I, Enri LR, Torta R, De Luca A, et al. Development of standard operating protocols for the optimization of cannabis-based formulations for medical purposes. Front Pharmacol. 2019;10:701.

47. Gould J. The cannabis crop. Nature. 2015;525(7570):S2-3.

48. Ben-Shabat S, Fride E, Sheskin T, Tamiri T, Rhee MH, Vogel $\mathrm{Z}$, et al. An entourage effect: inactive endogenous fatty acid glycerol esters enhance 2-arachidonoyl-glycerol cannabinoid activity. Eur J Pharmacol. 1998;353(1):23-31.

49. Russo EB, Marcu J. Cannabis pharmacology: the usual suspects and a few promising leads. Adv Pharmacol. 2017;80:67-134. 
50. Russo EB. The case for the entourage effect and conventional breeding of clinical cannabis: no "Strain", no gain. Front Plant Sci. 2019;9:1969.

51. Namdar D, Anis O, Poulin P, Koltai H. Chronological review and rational and future prospects of cannabis-based drug development. Molecules. 2020;25(20):4821.

52. Matarazzo AP, Elisei LMS, Carvalho FC, Bonfilio R, Ruela ALM, Galdino G, et al. Mucoadhesive nanostructured lipid carriers as a cannabidiol nasal delivery system for the treatment of neuropathic pain. Eur J Pharm Sci. 2021;159:105698.

53. Capano A, Weaver R, Burkman E. Evaluation of the effects of CBD hemp extract on opioid use and quality of life indicators in chronic pain patients: a prospective cohort study. Postgrad Med. 2020;132(1):56-61.

54. Maayah ZH, Takahara S, Ferdaoussi M, Dyck JRB. The molecular mechanisms that underpin the biological benefits of full-spectrum cannabis extract in the treatment of neuropathic pain and inflammation. Biochim Biophys Acta, Mol Basis Dis. 2020;1866(7):165771

55. Citti C, Russo F, Sgrò S, Gallo A, Zanotto A, Forni F, et al. Pitfalls in the analysis of phytocannabinoids in cannabis inflorescence. Anal Bioanal Chem. 2020;412(17):4009-22.

56. Brighenti V, Protti M, Anceschi L, Zanardi C, Mercolini L, Pellati $\mathrm{F}$. Emerging challenges in the extraction, analysis and bioanalysis of cannabidiol and related compounds. J Pharm Biomed Anal. 2021;192:113633.

57. Bekanntmachung zum Deutschen Arzneibuch 2018; Cannabisblüten; 24.04.2018; BAnz AT 24. Apr. 2018 B5; S 1-9

58. Brighenti V, Pellati F, Steinbach M, Maran D, Benvenuti S. Development of a new extraction technique and HPLC method for the analysis of non-psychoactive cannabinoids in fibre-type Cannabis sativa L. (hemp). J Pharm Biomed Anal. 2017;143:228-36.

59. E.C. Union method for the quantitative determination of the $\Delta 9$ - tetrahydrocannabinol content in hemp varieties. Delegated Regulation (EU) No 639/2014, Annex III as amended by Regulation (EU) 2017/1155. Official Journal of the European Union 30.6.2017;167:1-15. http:// data.europa.eu/eli/reg_del/2017/ 1155/oj. Accessed 15 Mar 2021.

60. Baldino L, Scognamiglio M, Reverchon E. Supercritical fluid technologies applied to the extraction of compounds of industrial interest from Cannabis sativa $\mathrm{L}$. and to their pharmaceutical formulations: a review. J Supercrit Fluids. 2020;165:104960.

61. Casiraghi A, Roda G, Casagni E, Cristina C, Musazzi UM, Franze $\mathrm{S}$, et al. Extraction method and analysis of cannabinoids in cannabis olive oil preparations. Planta Med. 2018;84(4):242-9.

62. Bekanntmachung zum Deutschen Arzneibuch 2020; Eingestellter Cannabisextrakt; 5.03.2020; BAnz AT 18.03.2020 B1; S1-4

63. Manns D, Norwig J, Reh K. Cannabis für medizinische Zwecke: Entwicklung von Arzneibuchmonographien als Qualitätsstandard. Bundesgesundheitsblatt Gesundheitsforschung Gesundheitsschutz. 2019;62(7):806-10.

64. Office of Medical Cannabis, MoH, Welfare and Sport, Netherlands. Information for pharmacists and healthcare professionals; 2021. https://english.cannabisbureau.nl/doctor-and-pharmacists. Accessed 15 Mar 2021.

65. Italian Health Ministry. Raccomandazioni per il medico prescrittore di sostanza vegetale Cannabis FM-2 infiorescenze. [Recommendations for the prescriber of plant substance Cannabis FM-2 inflorescences] 2017. https://www.salute.gov.it/imgs/C_17_pagin eAree_4589_listaFile_itemName_2_file.pdf [Italian]. Accessed 21 Mar 2021.

66. Brunetti P, Pichini S, Pacifici R, Busardò FP, Del Rio A. Herbal preparations of medical cannabis: a vademecum for prescribing doctors. Medicina (Kaunas). 2020;56(5):237.
67. Hazekamp A, Bastola K, Rashidi H, Bender J, Verpoorte R. Cannabis tea revisited: a systematic evaluation of the cannabinoid composition of cannabis tea. J Ethnopharmacol. 2007;113(1):85-90.

68. Alves P, Amaral C, Teixeira N, Correia-da-Silva G. Cannabis sativa: much more beyond $\Delta(9)$-tetrahydrocannabinol. Pharmacol Res. 2020;157:104822.

69. Pavlovic R, Nenna G, Calvi L, Panseri S, Borgonovo G, Giupponi L, et al. Quality Traits of "cannabidiol oils": cannabinoids content, terpene fingerprint and oxidation stability of european commercially available preparations. Molecules. 2018;23(5):1230.

70. Ramella A, Roda G, Pavlovic R, Dei Cas M, Casagni E, Mosconi $\mathrm{G}$, et al. Impact of lipid sources on quality traits of medical cannabis-based oil preparations. Molecules. 2020;25(13):2986.

71. Calvi L, Pentimalli D, Panseri S, Giupponi L, Gelmini F, Beretta G, et al. Comprehensive quality evaluation of medical Cannabis sativa L. inflorescence and macerated oils based on HSSPME coupled to GC-MS and LC-HRMS (q-exactive orbitrap) approach. J Pharm Biomed Anal. 2018;150:208-19.

72. Ternelli M, Brighenti V, Anceschi L, Poto M, Bertelli D, Licata $\mathrm{M}$, et al. Innovative methods for the preparation of medical Cannabis oils with a high content of both cannabinoids and terpenes. J Pharm Biomed Anal. 2020;186:113296.

73. De Vita D, Madia VN, Tudino V, Saccoliti F, De Leo A, Messore A, et al. Comparison of different methods for the extraction of cannabinoids from cannabis. Nat Prod Res. 2020;34(20):2952-8.

74. Cas MD, Casagni E, Casiraghi A, Minghetti P, Fornasari DMM, Ferri F, et al. Phytocannabinoids profile in medicinal cannabis oils: the impact of plant varieties and preparation methods. Front Pharmacol. 2020;11:1752.

75. Bettiol A, Lombardi N, Crescioli G, Maggini V, Gallo E, Mugelli A, et al. Galenic preparations of therapeutic Cannabis sativa differ in cannabinoids concentration: a quantitative analysis of variability and possible clinical implications. Front Pharmacol. 2018;9:1543.

76. Deidda R, Avohou HT, Baronti R, Davolio PL, Pasquini B, Del Bubba $\mathrm{M}$, et al. Analytical quality by design: development and control strategy for a LC method to evaluate the cannabinoids content in cannabis olive oil extracts. J Pharm Biomed Anal. 2019;166:326-35.

77. Carcieri C, Tomasello C, Simiele M, De Nicolo A, Avataneo $\mathrm{V}$, Canzoneri L, et al. Cannabinoids concentration variability in cannabis olive oil galenic preparations. J Pharm Pharmacol. 2018;70(1):143-9.

78. Baratta F, Simiele M, Pignata I, Ravetto Enri L, D'Avolio A, Torta R, et al. Cannabis-based oral formulations for medical purposes: preparation, quality and stability. Pharmaceuticals. 2021;14(2): 171

79. Sarma ND, Waye A, Elsohly MA, Brown PN, Elzinga S, Johnson HE, et al. Cannabis inflorescence for medical purposes: USP considerations for quality attributes. J Nat Prod. 2020;83(4):1334-51.

80. Dutch Ministry of Health Welfare and Sport, Office of Medical Cannabis, The Netherlands. Medical cannabis information brochure for patients; 2019. https://english.cannabisbureau.nl/ medicinal-cannabis/documents/circulars/2018/02/20/patientsguide-medicinal-cannabis. Accessed 2 Mar 2021.

81. Bar-Sela G, Zalman D, Semenysty V, Ballan E. The effects of dosage-controlled cannabis capsules on cancer-related cachexia and anorexia syndrome in advanced cancer patients: pilot study. Integr Cancer Ther. 2019 18:1534735419881498.

82. Sengupta A, Roychoudhury SK. Triglyceride composition of Sesamum indicum seed oil. J Sci Food Agric. 1976;27(2):165-9.

83. Zgair A, Lee JB, Wong JCM, Taha DA, Aram J, Di Virgilio D, et al. Oral administration of cannabis with lipids leads to high 
levels of cannabinoids in the intestinal lymphatic system and prominent immunomodulation. Sci Rep. 2017;7(1):14542.

84. Zgair A, Wong JCM, Lee JB, Mistry J, Sivak O, Wasan KM, et al. Dietary fats and pharmaceutical lipid excipients increase systemic exposure to orally administered cannabis and cannabisbased medicines. Am J Transl Res. 2016;8(8):3448-59.

85. Tayo B, Taylor L, Sahebkar F, Morrison G. A phase I, open-label, parallel-group, single-dose trial of the pharmacokinetics, safety, and tolerability of cannabidiol in subjects with mild to severe renal impairment. Clin Pharmacokinet. 2020;59(6):747-55.

86. Taylor L, Gidal B, Blakey G, Tayo B, Morrison G. A phase I, randomized, double-blind, placebo-controlled, single ascending dose, multiple dose, and food effect trial of the safety, tolerability and pharmacokinetics of highly purified cannabidiol in healthy subjects. CNS Drugs. 2018;32(11):1053-67.

87. Taylor L, Crockett J, Tayo B, Morrison G. A phase 1, openlabel, parallel-group, single-dose trial of the pharmacokinetics and safety of cannabidiol (CBD) in subjects with mild to severe hepatic impairment. J Clin Pharmacol. 2019;59(8):1110-9.

88. Letendre P, Carley D, inventors; Pier Pharmaceuticals, assignee. Low dose cannabinoid medicaments patent WO2012068516. 2012.

89. Pellikaan HC, Vermeulen PS, Bender JCME, Woerlee GF, inventors; Echo Pharmaceuticals B.V., assignee. Dosage unit for sublingual, buccal or oral administration of water-insoluble pharmaceutically active substances, such as cannabinoids patent WO2008033024. 2008.

90. Klumpers LE, Beumer TL, van Hasselt JGC, Lipplaa A, Karger LB, Kleinloog HD, et al. Novel $\Delta 9$-tetrahydrocannabinol formulation $\mathrm{Namisol}^{\circledR}$ has beneficial pharmacokinetics and promising pharmacodynamic effects. Br J Clin Pharmacol. 2012;74(1):42-53.

91. Poyatos L, Pérez-Acevedo AP, Papaseit E, Pérez-Mañá C, Martin $\mathrm{S}$, Hladun $\mathrm{O}$, et al. Oral administration of cannabis and $\Delta-9$ tetrahydrocannabinol (THC) preparations: a systematic review. Medicina (Kaunas). 2020;56(6):309.

92. Ahmed AIA, van den Elsen GAH, Colbers A, van der Marck MA, Burger DM, Feuth TB, et al. Safety and pharmacokinetics of oral delta-9-tetrahydrocannabinol in healthy older subjects: a randomized controlled trial. Eur Neuropsychopharmacol. 2014;24(9):1475-82.

93. Schussel V, Kenzo L, Santos A, Bueno J, Yoshimura E, de Oliveira Cruz Latorraca C, et al. Cannabinoids for nausea and vomiting related to chemotherapy: overview of systematic reviews. Phytotherapy Res. 2018;32(4):567-76.

94. Loftsson T. Cyclodextrins in parenteral formulations. J Pharm Sci. 2021;110(2):654-64.

95. Peball M, Krismer F, Knaus H-G, Djamshidian A, Werkmann M, Carbone F, et al. Non-motor symptoms in Parkinson's disease are reduced by nabilone. Ann Neurol. 2020;88(4):712-22.

96. Viernstein H, Toegel S, Schueller R, inventors; AOP Orphan Pharmaceuticals AG, assignee. Fast disintegrating compositions comprising nabilone and randomly methylated $\beta$-cyclodextrin patent WO2012069591. 2012.

97. Kellett K, Duggan BM, Gilson MK. Facile synthesis of a diverse library of mono-3-substituted $\beta$-cyclodextrin analogues. Supramol Chem. 2019;31(4):251-9.

98. Kingsley K, Lee S, Greenbaum E, inventors; Vireo Health LLC, assignee. Cannabinoid formulations with improved solubility patent US20190030170. 2019.

99. Devinsky O, Cross JH, Laux L, Marsh E, Miller I, Nabbout R, et al. Trial of cannabidiol for drug-resistant seizures in the dravet syndrome. N Engl J Med. 2017;376(21):2011-20.

100. Devinsky O, Patel AD, Cross JH, Villanueva V, Wirrell EC, Privitera $\mathrm{M}$, et al. Effect of cannabidiol on drop seizures in the lennox-gastaut syndrome. N Engl J Med. 2018;378(20):1888-97.
101. Thiele EA, Marsh ED, French JA, Mazurkiewicz-Beldzinska M, Benbadis SR, Joshi C, et al. Cannabidiol in patients with seizures associated with Lennox-Gastaut syndrome (GWPCARE4): a randomised, double-blind, placebo-controlled phase 3 trial. Lancet. 2018;391(10125):1085-96.

102. Wright S, Wilkhu J, inventors; GW Research Limited, assignee. Oral cannabinoid formulations patent WO2018150182. 2018.

103. Wilkhu J, Bender J, inventors; GW Research Limited, assignee. Cannabinoid formulations patent WO2018002665. 2018.

104. Wilkhu J, Bender J, inventors; GW Research Limited, assignee. Oral pharmaceutical formulation comprising cannabinoids and poloxamer patent WO2019135077. 2019.

105. Nguyen LC, Yang D, Nicolaescu V, Best TJ, Ohtsuki T, Chen $\mathrm{S}-\mathrm{N}$, et al. Cannabidiol inhibits SARS-CoV-2 replication and promotes the host innate immune response. bioRxiv. 2021:432967.

106. Emanuele RM, Gordon TS, Williford T, Andres M, Andres P, inventors; Artelo Biosciences, Inc., assignee. New solid forms of cannabidiol and uses thereof patent US20190177258. 2019.

107. Center for Drug Evaluation and Research, Food and Drug Administration, Silver Spring, MD, USA. Guidance for industry. Regulatory classification of pharmaceutical co-crystals. 2018. https://www.fda.gov/downloads/Drugs/Guidances/. Accessed 19 Mar 2021.

108. Almansa C, Frampton CS, Vela JM, Whitelock S, Plata-Salamán CR. Co-crystals as a new approach to multimodal analgesia and the treatment of pain. J Pain Res. 2019;12:2679-89.

109. Koch N, Jennotte O, Gasparrini Y, Vandenbroucke F, Lechanteur A, Evrard B. Cannabidiol aqueous solubility enhancement: comparison of three amorphous formulations strategies using different type of polymers. Int J Pharm. 2020;589:119812.

110. De Vries JA, Fernandez Cid MV, Heredia Lopez AM, Eiroa Martinez CM, inventors; Echo Pharmaceuticals B.V., Neth. assignee. Compressed tablet containing cannabidiol, method for its manufacture and use of such tablet in oral treatment of psychosis or anxiety disorders patent WO2015065179A1. 2015.

111. De Vries JA, Fernandez Cid MV, Heredia Lopez AM, inventors; Echo Pharmaceuticals B.V., assignee. Granulate containing cannabinoid, method for its manufacture and oral dosage unit comprising such granulate patent US20150132400. 2015.

112. Jagtap P, Shoken D, Avidan- Shlomovich S, Salzman AL, inventors; Beetlebung Pharma Ltd., assignee. Preparation of cannabinoid derivatives and conjugates as neuroprotectants and for treating pain patent WO2019159168. 2019.

113. Jagtap P, Musa S, inventors; Beetlebung Pharma Ltd., assignee. Synthesis of cannabinoid compounds patent WO2020031179. 2020.

114. Salzman A, Flower K, Garner CE, Jagtap P, Musa S, inventors; Kalytera Therapeutics, Inc., assignee. Synthesis of cannabidiol prodrugs for treating various disorders patent WO2018096504. 2018.

115. Hershberger P, Arlen P, inventors; Diverse Biotech, Inc., assignee. Cannabinoid conjugate molecules patent WO2020263888. 2020.

116. Patrician A, Versic-Bratincevic M, Mijacika T, Banic I, Marendic M, Sutlović D, et al. Examination of a new delivery approach for oral cannabidiol in healthy subjects: a randomized, doubleblinded, placebo-controlled pharmacokinetics study. Adv Therapy. 2019;36(11):3196-210.

117. Washington ME, Reillo M, inventors; Poviva Tea, LLC, assignee. Food and beverage compositions infused with lipophilic active agents and methods of use thereof patent WO2015191728. 2015.

118. Docherty J, Bunka CA, inventors; Poviva Tea, LLC, assignee. Transdermal and/or dermal delivery of lipophilic active agents patent WO2019178360. 2019. 
119. Docherty J, Bunka CA, inventors; Poviva Corp., assignee. Nanoemulsion compositions comprising biologically active ingredients patent WO2020236798. 2020.

120. Fleck JD, Betti AH, da Silva FP, Troian EA, Olivaro C, Ferreira F, et al. Saponins from Quillaja saponaria and Quillaja brasiliensis: particular chemical characteristics and biological activities. Molecules (Basel, Switzerland). 2019;24(1):171.

121. Sacks H, Edvinsson T, inventors; Gelpell AG Satipharm AG, assignee. Oral solid cannabinoid formulations, methods for producing and using thereof patent WO2017137992. 2017.

122. Mitelpunkt A, Kramer U, Hausman Kedem M, Zilbershot Fink E, Orbach R, Chernuha V, et al. The safety, tolerability, and effectiveness of PTL-101, an oral cannabidiol formulation, in pediatric intractable epilepsy: a phase II, open-label, single-center study. Epilepsy Behav. 2019;98:233-7.

123. Atsmon J, Heffetz D, Deutsch L, Deutsch F, Sacks H. Single-dose pharmacokinetics of oral cannabidiol following administration of PTL101: a new formulation based on Gelatin Matrix Pellets technology. Clin Pharmacol Drug Dev. 2018;7(7):751-8.

124. Yeshurun M, Sagiv SP, inventors; Stero Biotechs Ltd., Israel. assignee. Cannabidiol for reducing a steroid dose and treating inflammatory and autoimmune diseases patent WO2017191630A1. 2017.

125. Andriotis EG, Monou PK, Louka A, Papaefstathiou E, Eleftheriadis GK, Fatouros DG. Development of food grade 3D printable ink based on pectin containing cannabidiol/cyclodextrin inclusion complexes. Drug Dev Ind Pharm. 2020:1569-1577.

126. Lv P, Zhang D, Guo M, Liu J, Chen X, Guo R, et al. Structural analysis and cytotoxicity of host-guest inclusion complexes of cannabidiol with three native cyclodextrins. J Drug Deliv Sci Technol. 2019;51:337-44.

127. da Silva AJ, dos Santos ES. Energetic and thermodynamical aspects of the cyclodextrins-cannabidiol complex in aqueous solution: a molecular-dynamics study. Eur Biophys J. 2020;49(7):571-89.

128. Williams NNB, Ewell TR, Abbotts KSS, Harms KJ, Woelfel KA, Dooley GP, et al. Comparison of five oral cannabidiol preparations in adult humans: pharmacokinetics, body composition, and heart rate variability. Pharmaceuticals. 2021;14(1):35.

129. Knaub K, Sartorius T, Dharsono T, Wacker R, Wilhelm M, Schoen C. A novel self-emulsifying drug delivery system (SEDDS) based on VESIsorb formulation technology improving the oral bioavailability of cannabidiol in healthy subjects. Molecules. 2019;24(16):2967.

130. MacLeman PDR, Mavlianov M, inventors; Auscann Group Holdings Ltd., assignee. Solid self-emulsifying pharmaceutical compositions comprising cannabinoids patent WO2020024009. 2020.

131. MacLeman PDR, Mavlianov M, inventors; Auscann Group Holdings Ltd., assignee. Free-flowing powder compositions for oral solid dosages containing cannabinoids patent WO2020024011. 2020.

132. Anastassov G, Changoer L, inventors; AXIM Biotechnologies, Inc., USA. assignee. Chewing gum composition comprising cannabinoids and opioid agonists and/or antagonists patent WO2018075665A1. 2018.

133. Changoer L, Anastassov G, inventors; AXIM Biotechnologies, Inc., USA. assignee. Chewing gum composition comprising cannabinoids and nicotine patent WO2017189375A1. 2017.

134. Van Damme PA, Anastassov GE, inventors; Mareda Holding $\mathrm{BV}$, Neth. assignee. Chewing gum compositions comprising cannabinoids patent WO2009120080A1. 2009.

135. Greenspoon A. Inventor orally administrable chewing gum formulation patent WO2018018152. 2018.
136. Navon N. The accordion pill: unique oral delivery to enhance pharmacokinetics and therapeutic benefit of challenging drugs. Ther Delivery. 2019;10(7):433-42.

137. McCartney F, Jannin V, Chevrier S, Boulghobra H, Hristov DR, Ritter N, et al. Labrasol ${ }^{\circledR}$ is an efficacious intestinal permeation enhancer across rat intestine: ex vivo and in vivo rat studies. $\mathrm{J}$ Control Release. 2019;310:115-26.

138. Navon N, Reinberg R, Yakovson Y, inventors; Intec Pharma Ltd., assignee. Oral gastroretentive formulations and uses thereof patent WO2018011798. 2018.

139. Hardy J, Haywood A, Gogna G, Martin J, Yates P, Greer R, et al. Oral medicinal cannabinoids to relieve symptom burden in the palliative care of patients with advanced cancer: a doubleblind, placebo-controlled, randomised clinical trial of efficacy and safety of 1:1 delta-9-tetrahydrocannabinol (THC) and cannabidiol (CBD). Trials. 2020;21(1):611.

140. Onaivi ES, Singh Chauhan BP, Sharma V. Challenges of cannabinoid delivery: how can nanomedicine help? Nanomedicine. 2020;15(21):2023-8.

141. Mishra V, Nayak P, Yadav N, Singh M, Tambuwala MM, Aljabali AAA. Orally administered self-emulsifying drug delivery system in disease management: advancement and patents. Expert Opin Drug Deliv. 2021;18(3):315-332.

142. Bernkop-Schnürch A, Müllertz A, Rades T. Self-emulsifying drug delivery systems (SEDDS) - the splendid comeback of an old technology. Adv Drug Deliv Rev. 2019;142:1-2.

143. Buya AB, Beloqui A, Memvanga PB, Préat V. Self-nano-emulsifying drug-delivery systems: from the development to the current applications and challenges in oral drug delivery. Pharmaceutics. 2020;12(12): $1-52$.

144. Benita S, Nassar T, inventors; Yissum Research Development Company of the Hebrew University of Jerusalem Ltd., assignee. Self-emulsifying drug delivery systems for delivery of lipophilic compounds patent WO2020212976. 2020.

145. Honarikhezrbeigi S, Silver J, inventors; CannTrust Inc., assignee. Cannabis-based self-emulsifying product patent WO2020118415. 2020.

146. Zhang K, Tan X, Yu C, Chang T, Jin Q, inventors; Hanyi BioTechnology (Beijing) Co., Ltd., assignee. Cannabidiol self-emulsifying drug delivery system, solid self-emulsifying preparation and preparation method thereof patent CN110742861. 2020.

147. Izgelov D, Shmoeli E, Domb AJ, Hoffman A. The effect of medium chain and long chain triglycerides incorporated in self-nano emulsifying drug delivery systems on oral absorption of cannabinoids in rats. Int J Pharm (Amsterdam, Neth). 2020;580:119201.

148. Izgelov D, Regev A, Domb AJ, Hoffman A. Using the absorption cocktail approach to assess differential absorption kinetics of cannabidiol administered in lipid-based vehicles in rats. Mol Pharm. 2020;17(6):1979-86.

149. Izgelov D, Freidman M, Hoffman A. Investigation of cannabidiol gastro retentive tablets based on regional absorption of cannabinoids in rats. Eur J Pharm Biopharm. 2020;152:229-35.

150. Izgelov D, Domb AJ, Hoffman A. The effect of piperine on oral absorption of cannabidiol following acute vs. chronic administration. Eur J Pharm Sci. 2020;148:105313.

151. Diorio C, inventor PharmaCannis Labs LLC, assignee. Selfemulsifying drug delivery system patent US20190015346. 2019.

152. Izgelov D, Davidson E, Barasch D, Regev A, Domb AJ, Hoffman A. Pharmacokinetic investigation of synthetic cannabidiol oral formulations in healthy volunteers. Eur J Pharm Biopharm. 2020;154:108-15.

153. Liu ZX, Artmann C. Relative bioavailability comparison of different coenzyme Q10 formulations with a novel delivery system. Altern Ther Health Med. 2009;15(2):42-6. 
154. Magdassi S, Larush L, Ezra R, inventors; Yissum Research Development Company of the Hebrew University of Jerusalem Ltd Canna Powder Ltd., assignee. Formulated cannabis oil powder by nanoemulsifycation, methods of producing and uses thereof patent WO2020035850. 2020.

155. Fathordoobady F, Sannikova N, Guo Y, Singh A, Kitts DD, Pratap-Singh A. Comparing microfluidics and ultrasonication as formulation methods for developing hempseed oil nanoemulsions for oral delivery applications. Sci Rep. 2021;11(1):72.

156. Eskens $\mathrm{O}, \mathrm{Amin} \mathrm{S}$. Challenges and effective routes for formulating and delivery of epidermal growth factors in skin care. Int $\mathbf{J}$ Cosmet Sci. 2021;43(2):123-30.

157. Ballan E, Grinberg M, inventors; Cannabics Pharmaceuticals Inc., assignee. Novel system and method for microbiome profiling and modulation by means of cannabis administration patent US20190134123. 2019.

158. Ballan E, Grinberg M, inventors; Cannabics Pharmaceuticals Inc., assignee. Method for sensitivity testing of cannabinoids on patient-derived tumor biopsies and CTCs patent WO2019043679. 2019.

159. Ballan E, Borochov I, inventors; Cannabics Pharmaceuticals Inc., assignee. Cannabinoid compositions, methods of manufacture and use thereof patent WO2017145160. 2017.

160. Zettl BH, inventor ZYUS Life Sciences Inc., assignee. Formulation for pain management patent WO2020223800. 2020.

161. del Río C, Navarrete C, Collado JA, Bellido ML, GómezCañas M, Pazos MR, et al. The cannabinoid quinol VCE-004.8 alleviates bleomycin-induced scleroderma and exerts potent antifibrotic effects through peroxisome proliferator-activated receptor- $\gamma$ and CB2 pathways. Sci Rep. 2016;6(1):21703.

162. Palomares B, Ruiz-Pino F, Navarrete C, Velasco I, Sánchez-Garrido MA, Jimenez-Jimenez C, et al. VCE-004.8, a multitarget cannabinoquinone, attenuates adipogenesis and prevents dietinduced obesity. Sci Rep. 2018;8(1):16092.

163. Díaz-Alonso J, Paraíso-Luna J, Navarrete C, Del Río C, Cantarero I, Palomares B, et al. VCE-003.2, a novel cannabigerol derivative, enhances neuronal progenitor cell survival and alleviates symptomatology in murine models of Huntington's disease. Sci Rep. 2016;6:29789.

164. Aguareles J, Paraíso-Luna J, Palomares B, Bajo-Grañeras R, Navarrete C, Ruiz-Calvo A, et al. Oral administration of the cannabigerol derivative VCE-003.2 promotes subventricular zone neurogenesis and protects against mutant huntingtin-induced neurodegeneration. Transl Neurodegener. 2019;8(1):9.

165. Burgaz S, García C, Gómez-Cañas M, Muñoz E, Fernández-Ruiz J. Development of an oral treatment with the PPAR- $\gamma$-acting cannabinoid VCE-003.2 against the inflammation-driven neuronal deterioration in experimental Parkinson's disease. Molecules. 2019;24(15):2702.

166. Rodríguez-Cueto C, Santos-García I, García-Toscano L, EspejoPorras F, Bellido M, Fernández-Ruiz J, et al. Neuroprotective effects of the cannabigerol quinone derivative VCE-003.2 in SOD1G93A transgenic mice, an experimental model of amyotrophic lateral sclerosis. Biochem Pharmacol. 2018;157:217-26.

167. Jadoon KA, Ratcliffe SH, Barrett DA, Thomas EL, Stott C, Bell JD, et al. Efficacy and safety of cannabidiol and tetrahydrocannabivarin on glycemic and lipid parameters in patients with type 2 diabetes: a randomized, double-blind, placebo-controlled, parallel group pilot study. Diabetes Care. 2016;39(10):1777-86.

168. Ahmed W, Katz S. Therapeutic use of cannabis in inflammatory bowel disease. Gastroenterol Hepatol (NY). 2016;12(11):668-79.

169. de Carvalho ACA, de Souza GA, de Marqui SV, Guiguer ÉL, Araújo AC, Rubira CJ, et al. Cannabis and canabidinoids on the inflammatory bowel diseases: going beyond misuse. Int J Mol Sci. 2020;21(8):2940.
170. Silcock A, Wilkhu J, inventors; GW Research Limited, assignee. Cannabinoid pharmaceutical formulations comprising $\mathrm{pH}$ dependant release polymer patent WO2020240184. 2020.

171. Friedman D, inventor Icdpharma Ltd., assignee. Colonic delivery of cannabinoids in solid solution compositions patent WO2019159174. 2019.

172. Van De Donk T, Niesters M, Kowal MA, Olofsen E, Dahan A, Van Velzen M. An experimental randomized study on the analgesic effects of pharmaceutical-grade cannabis in chronic pain patients with fibromyalgia. Pain. 2019;160(4):860-9.

173. Chamberland G, Campbell C, Ringuette R, Bassett JD, Yifrach-Stav O, Rackov A, et al., inventors; Tetra Bio-Pharma Inc., assignee. Cannabis compositions and methods patent WO2020124220. 2020.

174. Kinsey PS, Laurenzi B, Smutney CC, Adamo B, Guarneri J, inventors; MannKind Corporation, assignee. Dry powder inhaler patent WO2017132601. 2017.

175. Guarneri JJ, Smutney CC, inventors; MannKind Corporation, assignee. Inhalable dry powders patent WO2020198051. 2020.

176. Leone-Bay A, Wesner G, inventors; Receptor Life Sciences, Inc., assignee. Rapid onset and extended action plant-based and synthetic cannabinoid formulations patent WO2019071213. 2019.

177. Overhoff KA, Johnston KP, Tam J, Engstrom J, Williams RO III. Use of thin film freezing to enable drug delivery: a review. J Drug Delivery Sci Technol. 2009;19(2):89-98.

178. Wang YB, Watts AB, Williams RO III. Effect of processing parameters on the physicochemical and aerodynamic properties of respirable brittle matrix powders. J Drug Delivery Sci Technol. 2014;24(4):390-6.

179. Watts AB, Wang Y-B, Johnston KP, Williams RO III. Respirable low-density microparticles formed in situ from aerosolized brittle matrices. Pharm Res. 2013;30(3):813-25.

180. Boylan NJ, Yucel T, Finnance SS, Zale SE, inventors; Molecular Infusions, LLC, assignee. Methods for non-irritating pulmonary administration of cannabinoids using soft mist inhalers patent WO2020167892. 2020.

181. Lam JKW, Cheung CCK, Chow MYT, Harrop E, Lapwood S, Barclay SIG, et al. Transmucosal drug administration as an alternative route in palliative and end-of-life care during the COVID-19 pandemic. Adv Drug Deliv Rev. 2020;160:234-43.

182. Madwar C, Paiement N, Obeid R, Conway JW, GonzalezLabrada E, inventors; IntelGenx Corp., assignee. Lipophilic active oral film formulation and method of making the same patent WO2020093146. 2020.

183. Fisher W, Katta S, Arlen P, inventors; Diverse Biotech, Inc., assignee. Cannabinoid preparations and therapeutic uses patent WO2019222459. 2019.

184. Lichtman AH, Lux EA, McQuade R, Rossetti S, Sanchez R, Sun W, et al. Results of a double-blind, randomized, placebocontrolled study of nabiximols oromucosal spray as an adjunctive therapy in advanced cancer patients with chronic uncontrolled pain. J Pain Symptom Manag. 2018;55(2):179.

185. Boland EG, Bennett MI, Allgar V, Boland JW. Cannabinoids for adult cancer-related pain: systematic review and metaanalysis. BMJ Support Palliat Care. 2020;10(1):14-24.

186. Carotenuto A, Costabile T, De Lucia M, Moccia M, Falco F, Petruzzo M, et al. Predictors of Nabiximols (Sativex ${ }^{\circledR}$ ) discontinuation over long-term follow-up: a real-life study. J Neurol. 2020;267(6): 1737-43.

187. Twelves C, Sabel M, Checketts D, Miller S, Tayo B, Jove $\mathrm{M}$, et al. A phase $1 \mathrm{~b}$ randomised, placebo-controlled trial of nabiximols cannabinoid oromucosal spray with temozolomide in patients with recurrent glioblastoma. Br J Cancer. 2021;124(8):1379-87. 
188. Überall MA. A review of scientific evidence for THC:CBD oromucosal spray (nabiximols) in the management of chronic pain. J Pain Res. 2020;13:399-410.

189. Itin C, Barasch D, Domb AJ, Hoffman A. Prolonged oral transmucosal delivery of highly lipophilic drug cannabidiol. Int $\mathbf{J}$ Pharm (Amsterdam, Neth). 2020;581:119276.

190. Hall SM, Vitetta L, Zhou Y, Rutolo DA, Jr., Coulson SM, inventors; Medlab Clinical U.S., Inc., USA. assignee. Transmucosal and transdermal delivery systems comprising nonionic surfactant and polyol patent WO2016141069A1. 2016.

191. Clarke SJ, Vitetta L, McLachlan AJ, Henson JD, Rutolo D, Hall $\mathrm{S}$. An oro-buccal nanoparticle delivered cannabis medicine for pain management in cancer: a clinical trial in progress. J Clin Oncol 2020;38:(suppl; abstr TPS12127)

192. Mabou Tagne A, Fotio Y, Lin L, Squire E, Ahmed F, Rashid TI, et al. Palmitoylethanolamide and hemp oil extract exert synergistic anti-nociceptive effects in mouse models of acute and chronic pain. Pharmacol Res. 2021;167:105545.

193. Kumar S, Garg SKR. Fast dissolving tablets (FDTs): current status, new market opportunities, recent advances in manufacturing technologies and future prospects. Int J Pharm Pharm Sci. 2014;6(7):22-35.

194. Seager H. Drug-delivery products and the Zydis fast-dissolving dosage form. J Pharm Pharmacol. 1998;50(4):375-82.

195. Bruun HZ, Boesen DS, Nielsen BP, inventors; Nordiccan A/S, assignee. An oral cannabinoid tablet comprising sugar alc. particles patent WO2020211915. 2020.

196. Brunn HZ, Boesen DS, Eriksen A, Wittorff H, inventors; MedCan Pharma A/S, assignee. Oral cannabinoid tablet patent US20200330423. 2020.

197. Bruun HZ, Boesen DS, Eriksen A, inventors; Nordiccan A/S, assignee. Cannabinoid lozenge controlled release formulation patent WO2020211911. 2020.

198. Dely AM, inventor Columbia Care, LLC, assignee. Sublingual and buccal dosage forms of cannabinoid extracts and method of use thereof patent WO2020097362. 2020.

199. Dely AM, Ben-Ari S, inventors; Columbia Care LLC, assignee. Topical formulations having cannabinoid patent WO2020142692. 2020.

200. Dely AM, Ben-Ari S, inventors; Columbia Care LLC, assignee. Cannabinoid rapidly disintegrating oral tablet patent WO2020146753. 2020.

201. Dely AM, Ben-Ari S, inventors; Columbia Care LLC, assignee. Inhalable dosage form of cannabinoid extract patent WO2020146383. 2020.

202. Changoer L, Anastassov G, inventors; Axim Biotechnologies, Inc., USA. assignee. Suppositories comprising cannabinoids patent WO2018075730A1. 2018.

203. Tanaka DS, inventor Transmucosal cannabinoid formulation including a chitosan excipient patent US20190000794. 2019.

204. Dely AM, inventor Columbia Care, LLC, assignee. Suppository formulations having cannabinoid patent WO2020097358. 2020.

205. Mercadante S, Vellucci R, Cuomo A, Adile C, Cortegiani A, Valle A, et al. Long-term efficacy and tolerability of intranasal fentanyl in the treatment of breakthrough cancer pain. Support Care Cancer. 2015;23(5):1349-54.

206. Splinter W. Novel approaches for treating pain in children. Curr Oncol Rep. 2019;21(2):11.

207. Selvaraj U, Woody DL, Boatright JH, Wen D, inventors; Stabilized solid nanoparticle formulations of cannabinoids and cannabinoid analogs with reduced Ostwald ripening for oral, inhalation, nasal and parenteral drug delivery patent WO2020186246. 2020.

208. Touitou E, Natsheh H, inventors; Yissum Research Development Company of the Hebrew University of Jerusalem Ltd., assignee. Cannabinoids compositions and methods patent WO2019106652. 2019.

209. Carpenter P, Deraney R, inventors; New Leaf Pharmaceuticals, LLC, assignee. Formulation for nasal delivery of cannabinoids patent US20200268708. 2020.

210. Cranford JA, Cooper DC, inventors; JC Pharma Inc., assignee. Method and composition for acute treatment of seizures including delta-9-tetrahydrocannabinol, cannabidiol and optionally terpenes patent US20190133993. 2019.

211. Leweke FM, Bryson N, Jr., inventors; Nasal cannabidiol compositions patent US20200170962. 2020.

212. Hommoss G, Pyo SM, Müller RH. Mucoadhesive tetrahydrocannabinol-loaded NLC-formulation optimization and long-term physicochemical stability. Eur J Pharm Biopharm. 2017;117:408-17.

213. Kováčik A, Kopečná M, Vávrová K. Permeation enhancers in transdermal drug delivery: benefits and limitations. Expert Opin Drug Deliv. 2020;17(2):145-55.

214. Hadley G, Derry S, Moore RA, Wiffen PJ. Transdermal fentanyl for cancer pain. Cochrane Database Syst Rev. 2013(10):CD010270.

215. Schmidt-Hansen M, Bromham N, Taubert M, Arnold S, Hilgart JS. Buprenorphine for treating cancer pain. Cochrane Database of Syst Rev. 2015(3): CD009596.

216. Gilron I, Baron R, Jensen T. Neuropathic pain: principles of diagnosis and treatment. Mayo Clin Proc. 2015;90(4):532-45.

217. Coderre TJ. Topical drug therapeutics for neuropathic pain. Expert Opin Pharmacother. 2018;19(11):1211-20.

218. Casiraghi A, Musazzi UM, Centin G, Franze S, Minghetti P. Topical administration of cannabidiol: influence of vehiclerelated aspects on skin permeation process. Pharmaceuticals. 2020;13(11):337.

219. Stinchcomb AL, Golinski MJ, Hammell DC, Howard JL, inventors; Alltranz, assignee. Products of tetrahydrocannabinol, compositions comprising prodrugs of tetrahydrocannabinol and methods of using the same patent US20090143462. 2009.

220. Doroudian A, Frankham P, inventors; Altum Pharmaceuticals Inc., assignee. Biphasix cannabinoid delivery patent WO2018213932. 2018.

221. Paudel KS, Hammell DC, Agu RU, Valiveti S, Stinchcomb AL. Cannabidiol bioavailability after nasal and transdermal application: effect of permeation enhancers. Drug Dev Ind Pharm. 2010;36(9):1088-97.

222. Osborne DW, Musakhanian J. Skin penetration and permeation properties of Transcutol ${ }^{\circledR}$-neat or diluted mixtures. AAPS PharmSciTech. 2018;19(8):3512-33.

223. Stinchcomb AL, Valiveti S, Hammell DC, Ramsey DR. Human skin permeation of $\Delta 8$-tetrahydrocannabinol, cannabidiol and cannabinol. J Pharm Pharmacol. 2004;56(3):291-7.

224. Stinchcomb AL, Banks SL, Golinski MJ, Howard JL, Hammell DC, inventors; AllTranz Inc., USA . assignee. Cannabidiol prodrugs in topical and transdermal administration with microneedles patent WO2011026144A1. 2011.

225. Stinchcomb AL, Nalluri BN, inventors; Alltranz, LLC, USA. assignee. Transdermal delivery of cannabinoids patent US20050266061A1. 2005.

226. Hammell DC, Zhang LP, Ma F, Abshire SM, McIlwrath SL, Stinchcomb AL, et al. Transdermal cannabidiol reduces inflammation and pain-related behaviours in a rat model of arthritis. Eur J Pain (UK). 2016;20(6):936-48.

227. Gutterman D, Sebree T, Smith T, Messenheimer J, inventors; Zynerba Pharmaceuticals, Inc., assignee. Synthetic transdermal cannabidiol for the treatment of focal epilepsy in adults patent US20190083388. 2019. 
228. Heussler H, Cohen J, Silove N, Tich N, Bonn-Miller MO, Du $\mathrm{W}$, et al. A phase 1/2, open-label assessment of the safety, tolerability, and efficacy of transdermal cannabidiol (ZYN002) for the treatment of pediatric fragile X syndrome. J Neurodev Disorders. 2019;11(1):16.

229. Bonn-Miller M, Tich N, Gutterman D, Messenheimer J, Sebree T, inventors; Zynerba Pharmaceuticals, Inc., assignee. Treatment of fragile X syndrome with cannabidiol patent US10213390. 2019.

230. Zieba J, Sinclair D, Sebree T, Bonn-Miller M, Gutterman D, Siegel S, et al. Cannabidiol (CBD) reduces anxiety-related behavior in mice via an FMRP1-independent mechanism. Pharmacol Biochem Behav. 2019;181:93-100.

231. Sebree T, Gutterman D, inventors; Zynerba Pharmaceuticals, Inc., assignee. Treatment of DiGeorge syndrome with cannabidiol patent WO2020121260. 2020.

232. Messenheimer J, Tich N, Gutterman D, Clauw D, inventors; Zynerba Pharmaceuticals, Inc., assignee. Methods of treatment of osteoarthritis with transdermal cannabidiol gel patent WO2019034985. 2019.

233. Cooper E, Callahan M, inventors; Botanix Pharmaceuticals Ltd. Botanix Pharmaceuticals, Inc., assignee. Formulations of cannabinoids comprising siloxane for the treatment of dermatitis and inflammatory skin diseases patent WO2018148785. 2018.

234. Aliyar H, Schalau G II. Recent developments in silicones for topical and transdermal drug delivery. Ther Deliv. 2015;6(7):827-39.

235. Schalau GK, Huber RO, Nartker LS, Thomas X. Novel siliconebased adhesive technology for transdermal therapy systems. Ther Deliv. 2017;8(4):175-8.

236. Sheriff $\mathrm{T}$, Lin MJ, Dubin D, Khorasani H. The potential role of cannabinoids in dermatology. J Dermatol Treat. 2020;31(8):839-45.

237. Chelliah MP, Zinn Z, Khuu P, Teng JMC. Self-initiated use of topical cannabidiol oil for epidermolysis bullosa. Pediatr Dermatol. 2018;35(4):e224-e227.

238. Hernández-Cervantes R, Méndez-Díaz M, Prospéro-García Ó, Morales-Montor J. Immunoregulatory role of cannabinoids during infectious disease. NeuroImmunoModulation. 2017;24(4-5):183-99.

239. Wong H, Hossain S, Cairns B, Long KA, Mancini A, inventors; Inmed Pharmaceuticals Inc., assignee. Topical formulations of cannabinoids for use in treatment of pain patent WO2019056123. 2019.

240. Hossain S, inventor InMed Pharmaceuticals Inc., assignee. Use of topical formulations of cannabinoids in the treatment of epidermolysis bullosa and related connective tissue disorders patent WO2017190249. 2017.

241. Maida V, Shi RB, Fazzari FGT, Zomparelli L. Topical cannabis-based medicines-a novel paradigm and treatment for non-uremic calciphylaxis leg ulcers: an open label trial. Int Wound J. 2020;17(5):1508-16.

242. Maida V. Medical cannabis in the palliation of malignant wounds - a case report. J Pain Symptom Manag. 2017;53(1):e4-6.

243. Maida V, Corban J. Topical medical cannabis: a new treatment for wound pain - three cases of pyoderma gangrenosum. J Pain Symptom Manag. 2017;54(5):732-6.

244. Sinai A, Turner Z, Baruch Y, inventors; One World Cannabis Ltd., assignee. Cannabis-based extracts and topical formulations for use in skin disorders patent US20180042890. 2018.

245. Wang MTM, Danesh-Meyer HV. Cannabinoids and the eye. Surv Ophthalmol. 2021;66(2):327-45.

246. Thapa D, Toguri JT, Szczesniak AM, Kelly ME. The nonpsychoactive phytocannabinoid, cannabidiol (CBD), and the synthetic derivatives, HU308 and CBD-DMH, reduces hyperalgesia and inflammation in a mouse model of corneal injury. FASEB J. 2017;31(S1):811.7.

247. Hanuš L, Breuer A, Tchilibon S, Shiloah S, Goldenberg D, Horowitz M, et al. HU-308: a specific agonist for CB2, a peripheral cannabinoid receptor. Proc Natl Acad Sci USA. 1999;96(25):14228-33.

248. Thapa D, Cairns EA, Szczesniak AM, Toguri JT, Caldwell MD, Kelly MEM. The cannabinoids $\triangle 8 \mathrm{THC}, \mathrm{CBD}$, and HU-308 act via distinct receptors to reduce corneal pain and inflammation. Cannabis Cannabinoid Res. 2018;3(1):11-20.

249. Taskar P, Adelli G, Patil A, Lakhani P, Ashour E, Gul W, et al. Analog derivatization of cannabidiol for improved ocular permeation. J Ocul Pharmacol Ther. 2019;35(5):301-10.

250. Kabiri M, Kamal SH, Pawar SV, Roy PR, Derakhshandeh M, Kumar U, et al. A stimulus-responsive, in situ-forming, nanoparticle-laden hydrogel for ocular drug delivery. Drug Deliv Transl Res. 2018;8(3):484-95.

251. Hossain S, Kabiri M, Yadav VG, inventors; InMed Pharmaceuticals Inc., assignee. Ocular drug delivery formulation comprising cellulosic polymer, anionic polysaccharide, nanoparticles comprising amphiphilic block copolymer, and cannabinoid patent WO2018205022. 2018.

252. Hsu E, Kumar U, Somvanshi RK, Zou S, inventors; InMed Pharmaceuticals Inc., assignee. Compositions comprising cannabinoids as neuroprotective agents for treating neurodegenerative diseases patent WO2020215164. 2020.

253. de Oliveira IF, Barbosa EJ, Peters MCC, Henostroza MAB, Yukuyama MN, Dos Santos Neto E, et al. Cutting-edge advances in therapy for the posterior segment of the eye: solid lipid nanoparticles and nanostructured lipid carriers. Int $\mathrm{J}$ Pharm. 2020;589:119831.

254. Taskar PS, Patil A, Lakhani P, Ashour E, Gul W, ElSohly MA, et al. $\Delta 9$-tetrahydrocannabinol derivative-loaded nanoformulation lowers intraocular pressure in normotensive rabbits. Transl Vis Sci Technol. 2019;8(5):15.

255. He X-L, Yang L, Wang Z-J, Huang R-Q, Zhu R-R, Cheng L-M. Solid lipid nanoparticles loading with curcumin and dexanabinol to treat major depressive disorder. Neural Regen Res. 2021;16(3):537-42.

256. Wolowich WR, Greif R, Kleine-Brueggeney M, Bernhard W, Theiler L. Minimal physiologically based pharmacokinetic model of intravenously and orally administered delta-9-tetrahydrocannabinol in healthy volunteers. Eur J Drug Metab Pharmacokinet. 2019;44(5):691-711.

257. Schindler EAD, Schnakenberg Martin AM, Sewell RA, Ranganathan M, DeForest A, Pittman BP, et al. In an exploratory randomized, double-blind, placebo-controlled, cross-over study, psychoactive doses of intravenous delta-9-tetrahydrocannabinol fail to produce antinociceptive effects in healthy human volunteers. Psychopharmacology (Heidelberg, Ger). 2020;237(10):3097-107.

258. Witowski PCG, Salm PJL, inventors; Nutrae, LLC, assignee. Encapsulated cannabinoid formulations for transdermal delivery patent WO2019140321. 2019.

259. Witowski CG, inventor Nutrae, LLC, assignee. Encapsulated cannabinoid formulations for inhalation or injection patent WO2019140325. 2019.

260. Salm JI, Witowski CG, inventors; Nutrae, LLC, assignee. Encapsulated cannabinoid formulations for oral delivery patent WO2019140323. 2019.

261. Wright S, Wilkhu J, inventors; GW Research Limited, assignee. Parenteral cannabinoid drug formulations patent WO2018002636. 2018.

262. Wright S, Wilkhu J, inventors; GW Research Limited, assignee. Parenteral cannabinoid formulations as neuroprotectant or anticonvulsive patent GB2551986. 2018. 
263. Lavasanifar A, Bolton AE, inventors; Cardiol Therapeutics Inc., assignee. Amphiphilic block copolymers, micelles, and methods for treating or preventing heart failure using a cardioactive agent patent WO2019113685. 2019.

264. Castor TP, Purdum G, inventors; Aphios Corporation, assignee. Nanoencapsulated delta-9-tetrahydrocannabinol patent US20120052119. 2012.

265. Knoeller I, Sowik T, inventors; SolMic Research GmbH, assignee. Stable cannabinoid compositions comprising micellar solution of poloxamer-based micelles encapsulating the cannabinoid patent WO2019008178. 2019.

266. Uziel A, Gelfand A, Amsalem K, Berman P, Lewitus GM, Meiri D, et al. Full-spectrum cannabis extract microdepots support controlled release of multiple phytocannabinoids for extended therapeutic effect. ACS Appl Mater Interfaces. 2020;12(21):23707-16.

267. Moura RP, Pacheco C, Pêgo AP, des Rieux A, Sarmento B. Lipid nanocapsules to enhance drug bioavailability to the central nervous system. J Control Release. 2020;322:390-400.

268. Aparicio-Blanco J, Sebastián V, Benoit JP, Torres-Suárez AI. Lipid nanocapsules decorated and loaded with cannabidiol as targeted prolonged release carriers for glioma therapy: in vitro screening of critical parameters. Eur J Pharm Biopharm. 2019;134:126-37.

269. Aparicio-Blanco J, Romero IA, Male DK, Slowing K, GarciaGarcia L, Torres-Suarez AI. Cannabidiol enhances the passage of lipid nanocapsules across the blood-brain barrier both in vitro and in vivo. Mol Pharm. 2019;16(5):1999-2010.

270. Ligresti A, Moriello AS, Starowicz K, Matias I, Pisanti S, De Petrocellis L, et al. Antitumor activity of plant cannabinoids with emphasis on the effect of cannabidiol on human breast carcinoma. J Pharmacol Exp Ther. 2006;318(3):1375.

271. Fraguas-Sanchez AI, Fernandez-Carballido A, SimancasHerbada R, Martin-Sabroso C, Torres-Suarez AI. CBD loaded microparticles as a potential formulation to improve paclitaxel and doxorubicin-based chemotherapy in breast cancer. Int $\mathbf{J}$ Pharm (Amsterdam, Neth). 2020;574:118916.

272. Thorat AA, Dalvi SV. Liquid antisolvent precipitation and stabilization of nanoparticles of poorly water soluble drugs in aqueous suspensions: recent developments and future perspective. Chem Eng J. 2012;181-182:1-34.

273. Štukelj R, Benčina M, Fanetti M, Valant M, Drab M, Iglĭ A, et al. Synthesis of stable cannabidiol (CBD) nanoparticles in suspension. Materiali in Tehnologije. 2019;53(4):543-9.

274. Gazendam A, Nucci N, Gouveia K, Abdel Khalik H, Rubinger L, Johal H. Cannabinoids in the management of acute pain: a systematic review and meta-analysis. Cannabis Cannabinoid Res. 2020;5(4):290-7.

275. Johal H, Devji T, Chang Y, Simone J, Vannabouathong C, Bhandari M. Cannabinoids in chronic non-cancer pain: a systematic review and meta-analysis. Clin Med Insights Arthritis Musculoskelet Disord. 2020;13:1179544120906461.

276. Almog S, Aharon-Peretz J, Vulfsons S, Ogintz M, Abalia H, Lupo T, et al. The pharmacokinetics, efficacy, and safety of a novel selective-dose cannabis inhaler in patients with chronic pain: a randomized, double-blinded, placebo-controlled trial. Eur J Pain (Oxford, UK). 2020;24(8):1505-16.

277. Chang-Douglass S, Mulvihill C, Pilling S. Cannabis-based medicinal products: summary of NICE guidance. BMJ. 2020;369:m1108.

278. Okusanya BO, Asaolu IO, Ehiri JE, Kimaru LJ, Okechukwu A, Rosales C. Medical cannabis for the reduction of opioid dosage in the treatment of non-cancer chronic pain: a systematic review. Syst Rev. 2020;9(1):167.
279. Barry AR, Chris CE. Treatment of chronic noncancer pain in patients on opioid therapy in primary care: a retrospective cohort study. Can Pharm J (Ott). 2020;153(1):52-8.

280. Inglet S, Winter B, Yost SE, Entringer S, Lian A, Biksacky M, et al. Clinical data for the use of cannabis-based treatments: a comprehensive review of the literature. Ann Pharmacother. 2020;54(11):1109-43.

281. Mohiuddin MM, Mizubuti GB, Haroutounian S, Smith SM, Rice ASC, Campbell F, et al. Adherence to Consolidated Standards of Reporting Trials (CONSORT) guidelines for reporting safety outcomes in trials of medical cannabis and cannabis-based medicines for chronic noncancer pain: a systematic review. Clin $\mathrm{J}$ Pain. 2020;36(4):302-19.

282. Chaves C, Bittencourt PCT, Pelegrini A. Ingestion of a THCrich cannabis oil in people with fibromyalgia: a randomized, double-blind, placebo-controlled clinical trial. Pain Med. 2020;21(10):2212-8.

283. Sawtelle L, Holle LM. Use of cannabis and cannabinoids in patients with cancer. Ann Pharmacother. 2021;55(7):870-90.

284. Häuser W, Welsch P, Klose P, Radbruch L, Fitzcharles MA. Efficacy, tolerability and safety of cannabis-based medicines for cancer pain: a systematic review with meta-analysis of randomised controlled trials. Schmerz. 2019;33(5):424-36.

285. Teoh D, Smith TJ, Song M, Spirtos NM. Care after chemotherapy: peripheral neuropathy, cannabis for symptom control, and mindfulness. Am Soc Clin Oncol Educ Book. 2018;38:469-79.

286. Blanton HL, Brelsfoard J, DeTurk N, Pruitt K, Narasimhan M, Morgan DJ, et al. Cannabinoids: current and future options to treat chronic and chemotherapy-induced neuropathic pain. Drugs. 2019;79(9):969-95.

287. Meng H, Page MG, Ajrawat P, Deshpande A, Samman B, Dominicis M, et al. Patient-reported outcomes in those consuming medical cannabis: a prospective longitudinal observational study in chronic pain patients. Can J Anesth. 2021;68:633.

288. Mücke M, Weier M, Carter C, Copeland J, Degenhardt L, Cuhls $\mathrm{H}$, et al. Systematic review and meta-analysis of cannabinoids in palliative medicine. J Cachexia Sarcopenia Muscle. 2018;9(2):220-34.

289. Wahby S, Karnik V, Brobbey A, Wiebe S, Sajobi T, Josephson CB. Cannabis use is both independently associated with and mediates worse psychosocial health in patients with epilepsy. $\mathbf{J}$ Neurol Neurosurg Psychiatry. 2019;90(8):945-51.

290. Calcaterra SL, Burnett-Hartman AN, Powers JD, Corley DA, McMullen CM, Pawloski PA, et al. A population-based survey to assess the association between cannabis and quality of life among colorectal cancer survivors. BMC Cancer. 2020;20(1):373.

291. Zhang K, Tan X, Chang T, Jin Q, inventors; Hanyi Biotechnology Beijing Co., Ltd., Peop. Rep. China. assignee. Composition containing cannabidiol and application patent CN107952075A. 2018.

292. Argueta DA, Ventura CM, Kiven S, Sagi V, Gupta K. A balanced approach for cannabidiol use in chronic pain. Front Pharmacol. 2020;11:561

293. Gulbransen G, Xu W, Arroll B. Cannabidiol prescription in clinical practice: an audit on the first 400 patients in New Zealand. BJGP Open. 2020;4(1):bjgpopen20X101010.

294. Rabgay K, Waranuch N, Chaiyakunapruk N, Sawangjit R, Ingkaninan K, Dilokthornsakul P. The effects of cannabis, cannabinoids, and their administration routes on pain control efficacy and safety: a systematic review and network meta-analysis. J Am Pharm Assoc. 2020;60(1):225-34.e6.

295. Rouhollahi E, Macleod BA, Barr AM, Puil E. Cannabis extract CT-921 has a high efficacy-adverse effect profile in a neuropathic pain model. Drug Des Dev Ther. 2020;14:3351-61.

296. Azmana M, Mahmood S, Hilles AR, Mandal UK, Saeed Al-Japairai KA, Raman S. Transdermal drug delivery system through 
polymeric microneedle: a recent update. J Drug Deliv Sci Technol. 2020;60:10187757.

297. Öblom H, Cornett C, Bøtker J, Frokjaer S, Hansen H, Rades T, et al. Data-enriched edible pharmaceuticals (DEEP) of medical cannabis by inkjet printing. Int J Pharm. 2020;589:119866.

298. Lim CBS, Sunderland VB, Lee YHE, inventors; IX Biopharma Ltd., assignee. Fast dissolving solid dosage form patent WO2014057351. 2014.

299. Hosseini A, McLachlan AJ, Lickliter JD. A phase I trial of the safety, tolerability and pharmacokinetics of cannabidiol administered as single-dose oil solution and single and multiple doses of a sublingual wafer in healthy volunteers. Br J Clin Pharmacol. 2021;87(4):2070-7.

300. Hua S. Advances in nanoparticulate drug delivery approaches for sublingual and buccal administration. Front Pharmacol. 2019;10:1328.

301. Davis A, inventor Futura Medical Developments Limited, assignee. Topical pharmaceutical formulation patent WO2016132159. 2016.

302. Lichtman AH, Lux EA, McQuade R, Rossetti S, Sanchez R, Sun W, et al. Results of a double-blind, randomized, placebocontrolled study of nabiximols oromucosal spray as a adjunctive therapy in advanced cancer patients with chronic uncontrolled pain. J Pain Symptom Manag. 2017;55:179-88.
303. Fallon MT, Albert Lux E, McQuade R, Rossetti S, Sanchez R, Sun W, et al. Sativex oromucosal spray as adjunctive therapy in advanced cancer patients with chronic pain unalleviated by optimized opioid therapy: two double-blind, randomized, placebocontrolled phase 3 studies. Br J Pain. 2017;11(3):119-33.

304. Mamber SW, Gurel V, Lins J, Ferri F, Beseme S, McMichael J. Effects of cannabis oil extract on immune response gene expression in human small airway epithelial cells (HSAEpC): implications for chronic obstructive pulmonary disease (COPD). J Cannabis Res. 2020;2(1):5.

305. Zerbe HG, Paiement N, inventors; IntelGenx Corp., assignee. Oral mucoadhesive dosage form patent US20110028431. 2011.

306. Jaervinen T, Mannila J, Jarho P, inventors; Novel methylated cyclodextrin complexes, treating a cannabinoid-responsive condition, and soluble dosage forms patent WO2003070774. 2003.

307. Atsmon J, Cherniakov I, Izgelov D, Hoffman A, Domb AJ, Deutsch L, et al. PTL401, a new formulation based on pro-nano dispersion technology, improves oral cannabinoids bioavailability in healthy volunteers. J Pharm Sci. 2018;107(5):1423-9.

308. Ringuette R, Campbell C, inventors; Tetra Bio-Pharma Inc., assignee. Compositions comprising cannabinoids and terpenes useful in the treatment of cancer and vascular ocular disorders via inhibition of hedgehog signalling patent WO2018205038. 2018. 